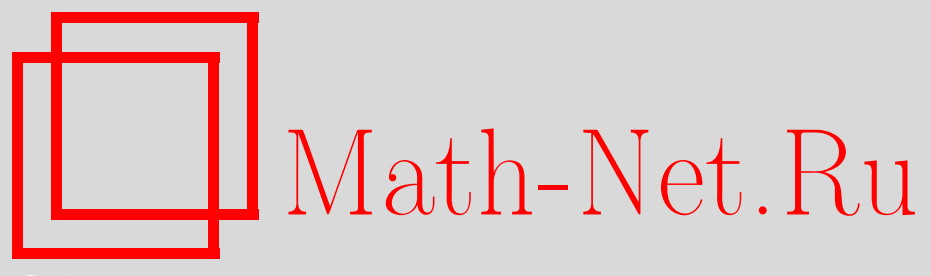

А. Г. Басуев, Модель Изинга в полупространстве. Серия фазовых переходов при малых магнитных полях, $Т M \Phi$, 2007, том 153, номер 2, 220-261

DOI: https://doi.org/10.4213/tmf6136

Использование Общероссийского математического портала Math-Net.Ru подразумевает, что вы прочитали и согласны с пользовательским соглашением http://www . mathnet.ru/rus/agreement

Параметры загрузки:

IP : 54.224 .60 .19

26 апреля 2023 г., $17: 12: 10$

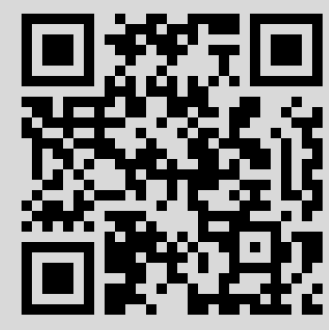




\title{
МОДЕЛЬ ИЗИНГА В ПОЛУПРОСТРАНСТВЕ. СЕРИЯ ФАЗОВЫХ ПЕРЕХОДОВ ПРИ МАЛЫХ МАГНИТНЫХ ПОЛЯХ
}

\begin{abstract}
Для модели Изинга в полупространстве при низких температурах и "неустойчивом граничном условии" доказано, что при каждом значении внешнего магнитного поля $\mu$ существует такой слой спинов, примыкающих к подложке, толщиной $q(\mu)$, что внутри этого слоя среднее значение спина близко к -1 , вне этого слоя близко к +1 . При уменьшении $\mu$ в точках $\mu_{q}$ толщина слоя $(-1)$-спинов меняется скачком на единицу, причем $q(\mu) \rightarrow \infty$ при $\mu \rightarrow+0$. В точках $\mu_{q}$ разрыва $q(\mu)$ сосуществуют две поверхностные фазы. Поверхностная свободная энергия кусочно-аналитична в области $\operatorname{Re} \mu>0$ и при низких температурах. Рассмотрена модель Изинга в полупространстве с произвольным внешним полем в нулевом слое и исследована соответствующая фазовая диаграмма. Доказано правило Антонова. Построено уравнение состояния в низших порядках с точностью до $x^{7}, x=e^{-2 \varepsilon}$, в частности, с этой точностью найдены точки сосуществования фаз $0,1,2$ и фаз $0,2,3$, где номер фазы соответствует высоте слоя неустойчивых спинов над подложкой.
\end{abstract}

Ключевые слова: многофазная контурная модель, гамильтониан границы, кластерное разложение гамильтониана границы, поверхностное натяжение, фазовая диаграмма поверхностных фаз, фазовый переход полного и пластичного смачивания.

Посвящается Роланду Львовичу Добрушину

\section{1. ВВЕДЕНИЕ}

Одна из идей классической работы Гиббса по термодинамике [1] заключалась в использовании одной и то же техники при рассмотрении как объемных, так и поверхностных фаз. Можно надеяться, что настоящая работа, в которой математически последовательно рассмотрены поверхностные фазовые переходы первого рода, является шагом в этом же направлении.

В двумерной модели Изинга поверхность раздела объемных фаз не является устойчивой при нулевом внешнем поле (см. работы [2] и [3]), в трехмерном слу-

${ }^{*}$ Санкт-Петербургский государственный университет технологии и дизайна, Санкт-Петербург, Россия. E-mail: Basuev@math.sutd.ru 
чае ситуация противоположная. Первый математический результат, доказывающий существование термодинамически устойчивой поверхности раздела объемных фаз трехмерной модели Изинга во всем пространстве, был получен Добрушиным [4]. Работа [4] является развитием метода Пайерлса и использует инвариантность гамильтониана относительно трансляций вдоль "вертикального направления". В работах [5]-[9] результаты Добрушина непосредственно обобщены на модель ВидомаРоулингсона. В работе [9] используется теория Пирогова-Синая [10] при изучении поверхностного натяжения и при рассмотрении поверхностных фаз. В работе [11] для повторения основного результата работы [4] использовались корреляционные неравенства, полученные Лебовицем [12] (см. также главу 5 в монографии [13]). Первая модель в полупространстве, для которой доказано существование фазового перехода в области единственности объемных фаз, была построена, насколько известно автору, в [14]. В цикле работ [15]-[20] техника корреляционных неравенств в основном использовалась для анализа модели Изинга в полупространстве. В настоящей работе мы приводим результаты, часть из которых была анонсирована в [21]. Сравнение результатов работ [5]-[9], [15]-[20] и настоящей работы требует отдельного более детального рассмотрения. Но надо все же отметить бо́льшую полноту полученных здесь результатов для модели Изинга в полупространстве по сравнению с названными работами.

В работе [22] были найдены первые шесть членов разложения поверхностной свободной энергии модели Изинга во всем пространстве. Модель Изинга в полупространстве и соответствующие фазовые переходы интенсивно рассматриваются в физической литературе с помощью методов среднего поля, различных приближений типа SOS-моделей (Solid-On-Solid), методов численного анализа [23], [24]. Для SOS-моделей в работах [25], [26] доказан результат о существовании поверхностных фазовых переходов, аналогичный полученному в настоящей работе для модели Изинга. Заметим, что, если в модели Изинга сделать разными горизонтальное и вертикальное взаимодействие спинов, а горизонтальную константу взаимодействия устремить к бесконечности, мы получим одну из SOS-моделей.

Результаты, связанные с фактом существования поверхностных фазовых переходов в полупространстве, оказались важны при нахождении области определения ренормгруппы [27]. В работе [28] показано, что проекция на двумерное пространство будет негиббсовой мерой, если при ненулевых внешних полях существует поверхностный фазовый переход.

Поверхностные явления изучались в работах [29]-[34]. В [31] развивается версия Заградника [35] теории Пирогова-Синая в применении к поверхностным фазам. Наше рассмотрение ближе к работе [31], где используется специальная модификация теории Пирогова-Синая для рассмотрения поверхностных фаз. Например, контурная модель с помеченными (labeled) контурами работы [31] соответствует многофазной контурной модели работ [36] с невзаимодействующими контурами. Отметим также работы [37]-[40], достаточно тесно примыкающие к настоящей работе.

Мы применяем теоремы III.1 и III.4 работ [36 $]^{1)}$ к изучению поверхностных фаз в модели Изинга в полупространстве, где нет инвариантности вдоль "вертикального 
направления" и необходимо использовать подходящую версию теории ПироговаСиная.

Полезно иметь в виду, что модель Изинга в полупространстве можно рассматривать как возмущение модели Изинга во всем пространстве. Это возмущение несимметрично относительно трансляций вдоль вертикального направления. Следовательно, вырожденная фазовая диаграмма поверхностных фаз во всем пространстве параметров (при нулевом магнитном поле сосуществуют поверхностные фазы Добрушина, отличающиеся лишь сдвигом вдоль вертикального направления) при возмущении, снимающем трансляционную инвариантность вдоль вертикального направления, должна преобразовываться в фазовую диаграмму без вырождения. Установить вид фазовой диаграммы с точностью до гомеоморфизма позволяют тонкие корреляционные неравенства Лебовица (см. [12]). Для модели Изинга в полупространстве стремление толщины слоя неустойчивых спинов к бесконечности при $\mu \rightarrow+0$ можно рассматривать как фазовый переход к полному смачиванию.

Качественная картина поведения модели следующая. При высоких температурах (энергия взаимодействия спинов $\varepsilon$ мала) поверхностная свободная энергия и корреляционные функции для модели в полупространстве являются гладкими функциями от $\varepsilon, \mu$ ( $\mu$ - внешнее поле) при любых граничных условиях. При низких температурах и "неустойчивом граничном условии" ("замороженные спины" подложки и внешнее поле направлены в противоположные стороны) для каждого $\mu$ существует слой спинов, примыкающих к подложке, толщиной $q(\mu)$ такой, что внутри этого слоя среднее значение спина близко к -1 ((-1)-“неустойчивые” спины), вне этого слоя среднее значение спина в каждой точке пространства близко к +1 . При уменьшении $\mu$ в точках $\mu_{q}$ толщина слоя неустойчивых спинов меняется скачком на единицу, $q(\mu) \rightarrow \infty$ при $\mu \rightarrow+0$. В точках $\mu_{q}$ разрыва $q(\mu)$ сосуществуют две поверхностные фазы. Кроме того, если перейти в систему отсчета, связанную с плоскостью, разделяющей $(+1)$ - и $(-1)$-спины, то при $\mu \rightarrow+0$ мы должны получить состояние Гиббса, описывающее термодинамически устойчивую поверхность раздела объемных фаз модели Изинга во всем пространстве и соответствующее соотношение (правило Антонова) для поверхностных свободных энергий. По существу, доказательство утверждений, приведенных выше, является основным результатом настоящей работы.

Построена сходящаяся схема расчета фазовой диаграммы модели Изинга в полупространстве, поверхностных термодинамических функций и их производных при низких температурах. Кроме этого, доказано, что поверхностная свободная энергия кусочно-аналитична в области $\operatorname{Re} \mu>0, \varepsilon>\varepsilon_{0}$, где $\varepsilon_{0}$ достаточно велико. Таким образом, поверхностная свободная энергия кусочно-аналитична по внешнему магнитному полю в той же области, что и объемная свободная энергия.

Введение произвольного магнитного поля в нулевом слое позволяет рассмотреть фазовый переход полного смачивания (см. [17], [18]). Подобный переход рассматривался в работах [41]-[45] (а также во многих других работах). С точки зрения

\footnotetext{
1) Ссылки на статьи [36] будут иметь следующий вид: раздел III.4 означает раздел 4 третьей работы в [36], (III.6) - формулу (6) третьей работы в [36] и т.п.
} 
полученных в настоящей работе результатов переход полного смачивания - это точка пространства параметров, в окрестности которой есть как устойчивые фазы с ограниченной толщиной слоя $(-1)$-спинов, так и устойчивые фазы с неограниченной толщиной слоя (-1)-спинов. В первом случае множество значений всех поверхностных натяжений имеет точку сгущения, значение в которой больше, чем поверхностное натяжение в системе. Во втором случае значение поверхностного натяжения в точке сгущения совпадает с поверхностным натяжением в системе.

Представим пространство $\mathbb{Z}^{\nu}, \nu \geqslant 3$, в виде объединения горизонтальных плоскостей (слоев): $\mathbb{Z}^{\nu} \bigcup_{k \in \mathbb{Z}} \mathbb{Z}_{k}^{\nu-1}, \mathbb{Z}_{+}^{\nu}:=\bigcup_{k \geqslant 0} \mathbb{Z}_{k}^{\nu-1} ; \mathbb{Z}^{\nu}$ и $\mathbb{Z}_{k}^{\nu-1}$ будем считать естественно вложенными в $\mathbb{R}^{\nu}$ и $\mathbb{R}_{k}^{\nu-1}$. Конфигурация спинов $\sigma$ - это функция на $\mathbb{Z}^{\nu}$, принимающая значения +1 или -1 . "Невозмущенные фазы" $\sigma^{q}, q=0,1, \ldots$, - это конфигурации спинов, равных -1 в $\bigcup_{k<q} \mathbb{Z}_{k}^{\nu-1}$ и равных +1 в $\bigcup_{k \geqslant q} \mathbb{Z}_{k}^{\nu-1}$. Пусть $\sigma_{1}$ - фиксированная конфигурация спинов. Статистическая сумма с граничным условием $\sigma_{1}$ определяется стандартным образом:

$$
\Xi\left(\Lambda \mid \sigma_{1}\right):=\sum_{\sigma: \sigma\left(\mathbb{Z}^{\nu} \backslash \Lambda\right)=\sigma_{1}\left(\mathbb{Z}^{\nu} \backslash \Lambda\right)} e^{-H_{\Lambda}\left(\sigma \mid \sigma_{1}\right)},
$$

где

$$
\begin{aligned}
H_{\Lambda}\left(\sigma \mid \sigma_{1}\right) & :=H\left(\sigma\left(\mathbb{Z}^{\nu}\right)\right)-H\left(\sigma_{1}\left(\mathbb{Z}^{\nu} \backslash \Lambda\right)\right)=-\frac{\varepsilon}{2} \sum_{[x, y] \cap \Lambda \neq \varnothing} \sigma_{x} \sigma_{y}-\frac{1}{2} \sum_{x \in \Lambda} \mu_{x} \sigma_{x}, \\
H(\sigma(\Lambda)) & :=-\frac{\varepsilon}{2} \sum_{[x, y] \subset \Lambda} \sigma_{x} \sigma_{y}-\frac{1}{2} \sum_{x \in \Lambda} \mu_{x} \sigma_{x}
\end{aligned}
$$

пара $[x, y]$ - ближайшие соседи в $\mathbb{Z}^{\nu}, \mu_{x}=\mu_{0}$ в слое $\mathbb{Z}_{0}^{\nu-1}$ и $\mu_{x}=\mu \geqslant 0$ в остальных слоях.

Пусть $\Lambda \subset \mathbb{Z}_{+}^{\nu}$ - цилиндр с основанием $\Lambda_{S} \subset \mathbb{Z}_{0}^{\nu-1}$ высоты $N,\left|\Lambda_{S}\right|$ - число узлов в $\Lambda_{S}, \sigma^{+}-$конфигурация спинов, равная +1 в $\mathbb{Z}^{\nu}$. Определим "поверхностные" статистические суммы

$$
\Xi_{q}^{N}\left(\Lambda_{S}\right):=\frac{e^{\varepsilon\left|\Lambda_{S}\right|} \Xi\left(\Lambda \mid \sigma^{q}\right)}{\Xi\left(\Lambda \mid \sigma^{+}\right)} .
$$

Поверхностную свободную энергию (поверхностное натяжение, если мы интерпретируем модель как газ частиц), соответствующую горизонтальной плоскости, определим следующим образом²):

$$
-p(\varepsilon, \mu):=-\lim _{\substack{N \rightarrow \infty, \Lambda_{S} \uparrow \mathbb{Z}_{0}^{\nu-1}}} \frac{1}{\left|\Lambda_{S}\right|} \ln \Xi_{q}^{N}\left(\Lambda_{S}\right) .
$$

Отметим, что каждой конфигурации $\sigma$, совпадающей вне некоторого $\Lambda$ с конфигурацией $\sigma^{q}$, соответствует лишь один бесконечный контур, разделяющий $(+1)-$ и

${ }^{2)}$ В стандартных физических обозначениях поверхностная свободная энергия равна $-p(\varepsilon, \mu)$. Так как далее будут использоваться одни и те же уравнения для объемных и поверхностных фаз, проще сохранить эти обозначения для соответствующих величин. 
$(-1)$-спины. Будем обозначать подобные контуры как $\Gamma_{\infty}^{q}$. Контур конфигурации $\sigma^{q}$ обозначим $\Gamma_{\infty}^{q, 0}-$ это плоскость на расстоянии $q-1 / 2$ над $\mathbb{Z}_{0}^{\nu-1}$.

Приведем нестрогие соображения, на основании которых найдем фазовую диаграмму модели Изинга в полупространстве с точностью до гомеоморфизма.

В статистических суммах $\Xi\left(\Lambda \mid \sigma^{q}\right)$ будем суммировать по простейшим конфигурациям, для которых поверхность, разделяющая $(+1)-$ и $(-1)$-спины, состоит лишь из одного контура $\Gamma_{\infty}^{q}$ (так называемое приближение сплошных фаз, в этом случае $\left.\Xi\left(\Lambda \mid \sigma^{+}\right)=e^{\mu|\Lambda| / 2}\right)$. Кроме того, будем считать, что каждая связная компонента проекции вертикальных граней $\Gamma_{\infty}^{q}$ на $\mathbb{Z}_{0}^{\nu-1}$ охватывает лишь один узел $\mathbb{Z}_{0}^{\nu-1}$. Иначе говоря, $\Gamma_{\infty}^{q}$ состоит из вертикальных столбцов с единичным основанием и высотой не более $-q$. Пренебрегая взаимодействием столбцов типа "твердой сердцевины" (оставляя в майеровском разложении лишь графы-точки) и учитывая, что "активность столбца" равна $e^{-2(\nu-1) \varepsilon\left|q_{1}\right|-\mu\left(q+q_{1}\right)}$, получаем из (1) и $(2)$, что

$$
\frac{1}{\left|\Lambda_{S}\right|} \ln \Xi_{q}^{N}\left(\Lambda_{S}\right) \approx p_{0}^{q}=-\mu q+\sum_{\substack{q_{1} \geqslant-q, q_{1} \neq 0}} e^{-2(\nu-1) \varepsilon\left|q_{1}\right|-\mu\left(q+q_{1}\right)} .
$$

Как известно из термодинамики, устойчива поверхностная фаза с минимальным поверхностным натяжением, таким образом, фазовая диаграмма задается функцией

$$
G^{0}(\varepsilon, \mu)=\left\{q:-p_{q}^{0}(\varepsilon, \mu)=\min _{q_{1}}\left(-p_{q_{1}}^{0}(\varepsilon, \mu)\right)\right\} .
$$

Используя явный вид $p_{q}^{0}$, можно убедиться, что

$$
G^{0}\left(\varepsilon, \mu_{q}^{0}(\varepsilon)\right)=\{q, q+1\}, \quad G^{0}(\varepsilon, \mu)=\{q\}, \quad \mu_{q}^{0}(\varepsilon)<\mu<\mu_{q+1}^{0}(\varepsilon),
$$

где функция $\mu_{q}^{0}(\varepsilon)$ определена неявно уравнением

$$
\mu_{q}^{0}=e^{-2(\nu-1) \varepsilon(q+1)}+\left(e^{-\mu_{q}^{0}}-1\right) \sum_{\substack{q_{1} \geqslant-q, q_{1} \neq 0}} e^{-2(\nu-1) \varepsilon\left|q_{1}\right|-\mu_{q}^{0}\left(q+q_{1}\right)} .
$$

\section{2. ОСНОВНЫЕ АЛГЕБРАИЧЕСКИЕ ПОСТРОЕНИЯ ДЛЯ МОДЕЛИ ИЗИНГА В $\mathbb{Z}_{+}^{\nu}$}

В модели Изинга удобно ввести следующие статистические суммы:

$$
\begin{gathered}
\Xi_{+}(\Lambda):=\frac{\Xi\left(\Lambda \mid \sigma^{+}\right)}{a_{\Lambda}}, \quad \Xi_{-}(\Lambda):=\frac{\Xi\left(\Lambda \mid \sigma^{-}\right)}{a_{\Lambda}}, \\
\Xi_{q}(\Lambda):=\frac{\Xi\left(\Lambda \mid \sigma^{q}\right)}{a_{\Lambda}},
\end{gathered}
$$

где $a_{\Lambda}:=\exp \left\{\sum_{[x, y] \cap \Lambda \neq \varnothing} \varepsilon / 2+\sum_{x \in \Lambda} \mu_{x} / 2\right\}, \sigma^{-}$- конфигурация спинов, равных -1 в $\mathbb{Z}^{\nu}$. Легко видеть, что статистические суммы $\Xi_{+}(\Lambda), \Xi_{-}(\Lambda)$ можно записать в виде статистических сумм "газа контуров с внешним полем":

$$
\Xi_{+}(\Lambda)=\sum_{X_{1} \subset \Lambda} F^{X_{1}} b^{V_{\mathrm{o}}\left(X_{1}\right)}, \quad \Xi_{-}(\Lambda)=\sum_{X_{1} \subset \Lambda} F^{X_{1}} b^{\Lambda \backslash V_{\mathrm{o}}\left(X_{1}\right)},
$$


где $X_{1}$ - набор непересекающихся контуров модели Изинга в $\Lambda$,

$$
b:=e^{-2 \mu}, \quad F^{X_{1}}:=\prod_{\Gamma \in X_{1}} F(\Gamma), \quad F(\Gamma):=e^{-\varepsilon|\Gamma|},
$$

$|\Gamma|$ - площадь поверхности $\Gamma, V_{\mathrm{o}}\left(X_{1}\right)$ - те узлы $\Lambda$, которые лежат внутри нечетного числа контуров $X_{1}$. Так как каждая конфигурация спинов, совпадающая вне $\Lambda$ с $\sigma^{q}$, задает ровно один контур $\Gamma_{\infty}^{q}$ с бесконечным носителем, статистическую сумму $\Xi_{q}(\Lambda)$ можно представить в виде

$$
\Xi_{q}(\Lambda)=\sum_{\Gamma_{\infty}^{q}: \Gamma_{\infty}^{q} \backslash \Gamma_{\infty}^{q, 0} \subset \Lambda} e^{-\varepsilon\left|\Gamma_{\infty}^{q} \cap \Lambda\right|} \Xi_{+}\left(\Lambda_{\uparrow}\left(\Gamma_{\infty}^{q}\right)\right) \Xi_{-}\left(\Lambda_{\downarrow}\left(\Gamma_{\infty}^{q}\right)\right),
$$

где $\Gamma_{\infty}^{q, 0}-$ контур конфигурации $\sigma^{q} ; \Lambda_{\uparrow}\left(\Gamma_{\infty}^{q}\right)$ и $\Lambda_{\downarrow}\left(\Gamma_{\infty}^{q}\right)-$ те точки $\Lambda$, где находятся соответственно $(+1)$-спины и $(-1)$-спины для конфигурации, соответствующей контуру $\Gamma_{\infty}^{q}$. Нам потребуются теоремы I.2, I.3 в частном случае модели Изинга. Переформулируем их в виде двух лемм.

ЛЕмма 1. Пусть функиионал $\mathcal{F}(\Gamma)$ определен рекуррентными соотношениями

$$
\mathcal{F}(\Gamma):=F(\Gamma) \sigma(V(\Gamma)), \quad \sigma(V):=b^{V} \sum_{X_{1} \subset V} F^{X_{1}} \widetilde{\varphi}_{X_{1}} \delta\left(\partial V \cup X_{1}\right),
$$

где $F, b$ определены выше, $X_{1}$ - набор непересекаюшихся контуров в $V$,

$$
\begin{gathered}
\widetilde{\varphi}_{X_{1}}:=\left(-b^{V(\Gamma)}\right)^{X_{1} \backslash \min X_{1}}\left(b^{-V(\Gamma)}-b^{V(\Gamma)}\right)^{\min X_{1}}, \\
\delta\left(\partial V \cup X_{1}\right):=\Xi_{+}^{-1}(V) \prod_{i \in l\left(\partial V \cup X_{1}\right)} \Xi_{+}\left(V_{i}\right),
\end{gathered}
$$

$\min X_{1}$ - те контуры $X_{1}$, внутри которых нет других контуров $X_{1}, l\left(\partial V \cup X_{1}\right)$ номера связных компонент, на которые $V$ разбито контурами $X_{1}$. Тогда для всех $V$ выполнены равенства

$$
\Xi_{+}(V)=\sum_{X_{1} \subset V} \mathcal{F}^{X_{1}}, \quad \sigma(V)=\frac{\Xi_{-}(V)}{\Xi_{+}(V)} .
$$

Лемма 2. Существуют константы $\varepsilon_{0}, C\left(C e^{-2 \nu \varepsilon_{0}}<1\right)$ такие, что при

$$
|F(\Gamma)|<e^{-\varepsilon_{0}|\Gamma|}, \quad\left|b^{2}\right|+C e^{-2 \nu \varepsilon_{0}}|1-b| \leqslant 1
$$

сходятся кластерные разложения

$$
\begin{gathered}
\ln \Xi_{+}(V)=\sum_{X \subset V}^{0} \varphi^{0}(X), \\
s(\mathcal{F}):=\lim _{V \uparrow \mathbb{Z}^{\nu}} \frac{1}{|V|} \ln \Xi_{+}(V)=\sum_{X: \min _{1} X=0}^{0} \varphi^{0}(X), \\
\ln \Delta(V)=\ln \left(\Xi_{+}(V) e^{-s|V|}\right)=-\sum_{X}^{0} \varphi^{0}(X) \frac{|V \cap V(X)|}{|V(X)|}, \\
\ln \delta\left(\partial V \cup X_{1}\right)=-\sum_{\substack{X \subset V, U_{0}\left(X, X_{1}\right) \neq 0}}^{0} \varphi^{0}(X),
\end{gathered}
$$


где $X$ - произвольный набор ${ }^{3)}$ контуров в $\mathbb{Z}^{\nu}, \varphi^{0}(X)$ - функиии Урселла, определяемые плотностями $\mathcal{F}^{X} e^{-U^{0}(X)}$ (см. раздел I.3), $\min _{1} V$ - лексикографический минимум $V$. Если $V$ - куб и $|\partial V|$ - площадь его поверхности, то верно разложение

$$
p_{s t}^{+}(\varepsilon, \mu):=-\lim _{V \uparrow \mathbb{Z}^{\nu}} \frac{1}{|\partial V|} \ln \left(\Xi_{+}(V) e^{-s|V|}\right)=\sum_{X: \min _{1} V(X) \cap \mathbb{Z}_{0}^{\nu-1}=0}^{0} \varphi^{0}(X) .
$$

Кроме того, функции, определеннье в (12) и (13), вещественно-аналитичны в области, заданной неравенствами (11), и непрерывны в ее замыкании.

В леммах 1 и 2 не используется явный вид $F(\Gamma)$. Подчеркнем, что второе неравенство (11) верно для всех $b \in[0,1]$, следовательно, внешнее поле произвольно.

Из формул (3), (8), (12) и (7) находим, что

$$
\begin{aligned}
\Xi_{q}^{N}\left(\Lambda_{S}\right)= & \sum_{\substack{\Gamma_{\infty}^{q}, X_{1}: \Gamma_{\infty}^{q} \backslash \Gamma_{\Gamma_{1}, 0}^{q, 0} \subset \Lambda, X_{1} \subset \Lambda_{\downarrow}\left(\Gamma_{\infty}^{q}\right)}} F^{X_{1}} b^{\Lambda_{\downarrow}\left(\Gamma_{\infty}^{q}\right)} \widetilde{\varphi}_{X_{1}} \times \\
& \quad \times \exp \left\{-\varepsilon\left(\left|\Gamma_{\infty}^{q} \cap \Lambda\right|-\left|\Lambda_{S}\right|\right)-\sum_{X \subset \Lambda: X \cap\left(\Gamma_{\infty}^{q} \cup X_{1}\right) \neq \varnothing}^{0} \varphi^{0}(X)\right\} .
\end{aligned}
$$

Представление (14) является основным для поверхностных статистических сумм. Ключевым для использования теоремы III.1 является определение границ, которое позволит рассматривать (14) как многофазную контурную модель, выписать гамильтониан границы, в том числе соответствующие контурные функционалы и их взаимодействие.

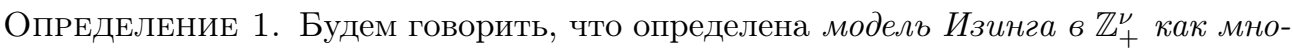
гофазная контурная модель в $\mathbb{Z}^{\nu-1}, \mu_{0}=\mu$, если задано следующее. Множество "названий фаз" есть $\mathbb{Z}_{+}=\{0,1, \ldots\}$. Множество границ $\gamma_{q}$ совпадает с множеством пар $\left(\Gamma_{\infty}^{q}, X_{1}\right)$ в $(14)$. Носитель $V_{\vec{\partial}}$ границы $\vec{\partial} \in \gamma_{q}$ включает в себя проекцию поверхностей $X_{1}$ на $\mathbb{R}_{0}^{\nu-1}\left(\operatorname{pr} X_{1} \subset \mathbb{R}_{0}^{\nu-1}\right)$, содержит проекции вертикальных граней $\Gamma_{\infty}^{q}$, а также проекции тех горизонтальных граней $\Gamma_{\infty}^{q}$, число которых в столбце над узлом $x \in \mathbb{Z}_{0}^{\nu-1}$ не менее трех (таким образом, $V_{\vec{\partial}}$ состоит из граней размерности $\nu-1$, $\nu-2)$. Связная компонента $V_{i}(\overrightarrow{\boldsymbol{\partial}})$ множества $\mathbb{R}_{0}^{\nu-1} \backslash V_{\vec{\partial}}$ "занята" фазой $k_{i}(\overrightarrow{\boldsymbol{\partial}})$, где $k_{i}(\overrightarrow{\boldsymbol{\partial}})$ - высота $\Gamma_{\infty}^{q}$ над $\Gamma_{\infty}^{0,0}$ в цилиндре $\operatorname{pr}^{-1} V_{i}(\overrightarrow{\boldsymbol{\partial}})$. Разложение границы на контуры соответствует разложению границы на связные компоненты.

Вместо контура $\Gamma_{\infty}^{q}$ удобно использовать, вообе говоря, несвязную поверхность

$$
\vec{\partial}_{0}:=\Gamma_{\infty}^{q} \backslash\left(\bigcup_{i} \operatorname{pr}^{-1} V_{i}(\vec{\partial})\right)
$$

\footnotetext{
3) Если в статсумме идет суммирование по непересекающимся контурам, то при формальном переразложении логарифма статсуммы и величин типа $s_{q}$ суммирование ведется по произвольным наборам контуров $X$, среди которых могут быть и повторяющиеся контуры. Для повторяющихся контуров в соответствующих разложениях необходимо добавить в знаменатель факториалы числа повторяющихся контуров. Сумма $\sum^{0}$ с верхним индексом 0 содержит в себе эти сомножители.
} 
где $\vec{\partial}=\left(\Gamma_{\infty}^{q}, \varnothing\right)$. Таким образом, $\vec{\partial}_{0}$ состоит из вертикальных граней $\Gamma_{\infty}^{q}$ и тех горизонтальных граней, число которых в столбце над узлом из $\mathbb{Z}_{0}^{\nu-1}$ не меньше трех.

Нам нужна будет следующая простая лемма.

Лемма 3. Связность $\vec{\partial}_{0}$ эквивалентна связности проекции $\vec{\partial}_{0}$ на $\mathbb{R}_{0}^{\nu-1}$. Справедливо неравенство

$$
\left|\Gamma_{\infty}^{q} \cap \Lambda\right|-\left|\Lambda_{S}\right|=\left|\vec{\partial}_{0}\right|_{1}-\left|V_{\overrightarrow{\boldsymbol{\partial}}_{0}}\right| \geqslant\left|\overrightarrow{\boldsymbol{\partial}}_{0}\right|_{1, \text { vert }}+\frac{2}{3}\left|\overrightarrow{\boldsymbol{\partial}}_{0}\right|_{1, \mathrm{hor}},
$$

где $\left|\overrightarrow{\boldsymbol{\partial}}_{0}\right|_{1}-$ число $(\nu-1)$-мерных граней $\overrightarrow{\boldsymbol{\partial}}_{0},\left|\overrightarrow{\boldsymbol{\partial}}_{0}\right|_{1, \text { vert (hor) }}-$ число $(\nu-1)$-мерных вертикальных (горизонтальных) граней $\overrightarrow{\boldsymbol{\partial}}_{0},\left|V_{\overrightarrow{\boldsymbol{\partial}}_{0}}\right|-$ число $(\nu-1)$-мерных граней $V_{\overrightarrow{\boldsymbol{\partial}}_{0}}$. Число поверхностей $\overrightarrow{\boldsymbol{\Gamma}}_{0}\left(V_{\overrightarrow{\boldsymbol{\Gamma}}_{0}}\right.$ свлзно, $\left.\left(\overrightarrow{\boldsymbol{\Gamma}}_{0}, \varnothing\right) \in \gamma_{q}\right)$ с заданной площадъю удовлетворяет неравенству

$$
\mid\left\{\overrightarrow{\boldsymbol{\Gamma}}_{0}: \min _{1} \overrightarrow{\boldsymbol{\Gamma}}_{0} \cap \Gamma_{\infty}^{q, 0}=0,\left|\overrightarrow{\boldsymbol{\Gamma}}_{0}\right|_{1}=\text { const }\right\} \mid \leqslant 3^{\left|\overrightarrow{\boldsymbol{\Gamma}}_{0}\right|_{1}}
$$

Используя определение 1, определение III.2 и сравнивая формулы (III.6), (III.7) и (14), получаем

$$
\Xi_{q}^{N}\left(\Lambda_{S}\right)=\sum_{\overrightarrow{\boldsymbol{\partial}} \in \gamma_{q}^{N}\left(\Lambda_{S}\right)} e^{-H_{f}^{\Lambda}(\overrightarrow{\boldsymbol{\partial}})},
$$

где $\gamma_{q}^{N}\left(\Lambda_{S}\right)$ - множество границ суммы в (14) (далее $\left.\overrightarrow{\boldsymbol{\partial}}=\left(\Gamma_{\infty}^{q}, X_{1}\right)\right)$,

$$
\begin{aligned}
& H_{f}^{\Lambda}(\overrightarrow{\boldsymbol{\partial}}):=H_{0}^{\Lambda}(\overrightarrow{\boldsymbol{\partial}})+\sum_{V: V \subset \mathbb{Z}_{0}^{\nu-1}} W^{\Lambda}(V \mid \overrightarrow{\boldsymbol{\partial}}) ; \\
& e^{-H_{0}^{\Lambda}(\overrightarrow{\boldsymbol{\partial}})}:=e^{-\varepsilon\left(\left|\overrightarrow{\boldsymbol{\partial}}_{0} \cap \Lambda\right|\right)-\left|V_{\overrightarrow{\boldsymbol{\partial}}_{0}}\right|} F^{X_{1}} b^{\Lambda}(\overrightarrow{\boldsymbol{\partial}}) \cap \mathrm{pr}^{-1} V_{\overrightarrow{\boldsymbol{\partial}}} \widetilde{\varphi}_{X_{1}} ; \\
& W^{\Lambda}(V \mid \overrightarrow{\boldsymbol{\partial}}):=\sum_{X: \operatorname{pr} X=V}^{0} W^{\Lambda}(X \mid \overrightarrow{\boldsymbol{\partial}}), \quad|V| \geqslant 2 ; \\
& W^{\Lambda}(X \mid \vec{\partial}):=\Theta_{\Lambda}(X \mid \vec{\partial}) \varphi^{0}(X) ; \\
& \Theta_{\Lambda}(X \mid \vec{\partial}):=\left\{\begin{array}{lll}
1, & X \cap\left(X_{1} \cup \Gamma_{\infty}^{q}\right) \neq \varnothing, & X \subset \Lambda, \\
0, & X \cap\left(X_{1} \cup \Gamma_{\infty}^{q}\right)=\varnothing ;
\end{array}\right. \\
& W^{\Lambda}(x \mid \overrightarrow{\boldsymbol{\partial}}):= \begin{cases}-k_{i}(\overrightarrow{\boldsymbol{\partial}}) \mu, & x \in V_{i}(\overrightarrow{\boldsymbol{\partial}}) \cap \Lambda, \\
0, & x \in\left(\mathbb{Z}_{0}^{\nu-1} \backslash \Lambda\right) \cup V_{\vec{\partial}}\end{cases}
\end{aligned}
$$

(здесь и далее $x, y$ - точки решетки $\mathbb{Z}^{\nu}$, а $X, Y$ - контуры модели Изинга).

Заметим, что при $\mu=0$ каждая граница имеет вид $\left(\Gamma_{\infty}^{q}, \varnothing\right)$, так как при $X_{1} \neq \varnothing$ величина $\left.\widetilde{\varphi}_{X_{1}}\right|_{\mu=0}=0$ (см. (9)). Гамильтониан взаимодействия контуров и величи́ны 
$h_{q}^{\Lambda}(x)$ можно записать также в виде

$$
\begin{gathered}
H_{1}^{\Lambda}(\overrightarrow{\boldsymbol{\partial}})=\sum_{X}^{0}\left[W^{\Lambda}(X \mid \overrightarrow{\boldsymbol{\partial}})-\sum_{q} \frac{\left|V_{q}(\overrightarrow{\boldsymbol{\partial}}) \cap \operatorname{pr} X\right|}{|\operatorname{pr} X|} W(X \mid q)\right] \\
h_{q}^{\Lambda}(x):=-q \mu+h_{q}^{1, \Lambda}(x)=-q \mu-\sum_{\substack{X \subset \Lambda, \min _{1}\left(X \cap \Gamma_{\infty}^{q, 0}\right)=x}}^{0} \varphi^{0}(X) .
\end{gathered}
$$

Положив

$$
\begin{aligned}
& h_{q}:=\left.h_{q}^{\Lambda}\right|_{\Lambda=\mathbb{Z}^{\nu}}, h_{q, N}:=\left.h_{q}^{\Lambda}\right|_{\Lambda=\cup_{0 \leqslant k \leqslant N-1}} \mathbb{Z}_{k}^{\nu-1}, \\
& z_{q}:=e^{h_{q}}, \quad z_{q, \Lambda}^{\Lambda_{S}}:=\exp \sum_{x \in \Lambda_{S}} h_{q}^{\Lambda}(x),
\end{aligned}
$$

получаем основное представление для статистических сумм (3), (14) с точностью до множителя $e^{c_{q}(\Lambda)}$, не влияющего на поверхностные термодинамические и корреляционные функции:

$$
\begin{gathered}
\Xi_{q}^{N}\left(\Lambda_{S}\right)=\sum_{\overrightarrow{\boldsymbol{\partial}} \in \gamma_{q}^{N}\left(\Lambda_{S}\right)} z_{q, \Lambda}^{\Lambda_{S}} z_{q, N}^{-V(\overrightarrow{\boldsymbol{\partial}})} e^{-H_{0}^{\Lambda}(\overrightarrow{\boldsymbol{\partial}})-H_{1}^{\Lambda}(\overrightarrow{\boldsymbol{\partial}})} \prod_{i \in l(\overrightarrow{\boldsymbol{\partial}})} z_{k_{i}(\overrightarrow{\boldsymbol{\partial}}), N}^{V_{i}(\overrightarrow{\boldsymbol{\partial}})}, \\
c_{q}^{\Lambda}:=\sum_{X}^{0}\left\{\Theta_{\Lambda}(X \mid q)-\frac{\left|\Lambda_{S} \cap \operatorname{pr} X\right|}{|\operatorname{pr} X|}\right\} \varphi^{0}(X) .
\end{gathered}
$$

Из леммы 2 вытекает, что величины (19)-(23) конечны при $N \rightarrow \infty$, причем величины (19)-(22) конечны при $N \rightarrow \infty, \Lambda_{S} \uparrow \mathbb{Z}_{0}^{\nu-1}$. Неравенства, доказываемые ниже в леммах 4-7, будут верны как при конечных, так и при бесконечных $\Lambda$, поэтому значок $\Lambda$ в дальнейшем будем опускать. Заметим, что условия 1 и 2 теоремы III.1 выполнены.

При $\mu=0, N \rightarrow \infty, q \rightarrow \infty$ (этот предельный переход рассмотрен Добрушиным в [4]) статистические суммы $(23)$ переходят в следующие $\left(\vec{\partial}=\left(\vec{\partial}_{0}, \varnothing\right)\right)$ :

$$
\begin{aligned}
& \Xi^{\mathrm{D}}\left(\Lambda_{S}\right):= \sum_{\overrightarrow{\boldsymbol{\partial}} \in \gamma_{0}\left(\Lambda_{S}\right)} \prod_{i \in \bar{l}\left(\overrightarrow{\boldsymbol{\partial}}_{0}\right)}\left(z_{k_{i}}^{\mathrm{D}}\right)^{V_{i}(\overrightarrow{\boldsymbol{\partial}}) \cap \Lambda_{S}} \times \\
& \times \exp \left\{-H_{0}(\overrightarrow{\boldsymbol{\partial}})-\sum_{X}^{0}\left[W(X \mid \overrightarrow{\boldsymbol{\partial}})-\sum_{q \in \mathbb{Z}} \frac{\left|V_{q}(\overrightarrow{\boldsymbol{\partial}}) \cap \operatorname{pr} X\right|}{|\operatorname{pr} X|} W(X \mid q)\right]\right\}, \\
& z_{q}^{\mathrm{D}}:=e^{h^{\mathrm{D}}}, \quad h^{\mathrm{D}}:=-\sum_{X: \min _{1}} \sum_{X \cap \mathbb{Z}_{0}^{\nu-1}=0}^{0} \varphi^{0}(X) .
\end{aligned}
$$

Данные статистические суммы описывают гиббсовское состояние, задающее термодинамически устойчивую поверхность раздела объемных $(+1)$ - и $(-1)$-фаз модели Изинга во всем пространстве при $\mu=0$.

В представлениях (14), (23) и в определении границ существенным является следующий факт. Возмущения вне контура $\Gamma_{\infty}^{q}$ "обрабатываются" разными способами. Для статистических сумм с устойчивым граничным условием используется 
сходящееся равномерно по магнитному полю низкотемпературное контурное разложение (12), а для статистических сумм с неустойчивым граничным условием представление (8). Таким образом, возмущения в статистических суммах с неустойчивым граничным условием непосредственно включаются в границу.

\section{3. ПРОВЕРКА СЛАБОСТИ ВЗАИМОДЕЙСТВИЯ КОНТУРОВ И УСЛОВИЯ ПАЙЕРЛСА МОДЕЛИ ИЗИНГА В $\mathbb{Z}_{+}^{\nu}$}

Проверим условия 3, 4 теоремы III.1 для величин $H_{0}(\overrightarrow{\boldsymbol{\partial}}), W(V \mid \overrightarrow{\boldsymbol{\partial}})$. Определим функции

$$
\begin{gathered}
h(X \mid \overrightarrow{\boldsymbol{\partial}}):=\sum_{k \in \mathbb{Z}} \Theta(X+k \vec{i} \mid \overrightarrow{\boldsymbol{\partial}}), \quad h(X):=\max _{q} h(X \mid q), \\
h(\overrightarrow{\boldsymbol{\partial}}):=\sum_{k \in \mathbb{Z}} \Theta\left(\Gamma_{0}+k \vec{i} \mid \overrightarrow{\boldsymbol{\partial}}\right),
\end{gathered}
$$

где $\vec{i}$ - единичный орт, перпендикулярный $\mathbb{Z}_{0}^{\nu-1}, \Gamma_{0}$ - контур модели Изинга в $\mathbb{Z}^{\nu}$ такой, что $V_{\overrightarrow{\boldsymbol{\partial}}} \subset \operatorname{pr} \Gamma_{0}$. Таким образом, $h(X)$ - это высота $X, h(\overrightarrow{\boldsymbol{\Gamma}})$ - высота контура $\Gamma_{\infty}^{q}(\overrightarrow{\boldsymbol{\Gamma}})$. Используя трансляционную инвариантность $\varphi^{0}(X)$, из $(21)$ имеем

$$
W(V \mid \overrightarrow{\boldsymbol{\partial}})=\sum_{\substack{X: \min _{\vec{i}} X=0, \operatorname{pr} X=V}}^{0} h(X \mid \overrightarrow{\boldsymbol{\partial}}) \varphi^{0}(X),
$$

где равенство $\min _{\vec{i}} X=0$ означает, что минимальная координата проекции $X$ на ось $\vec{i}$ равна нулю.

Лемма 4. Пусть $\vec{\partial} \in \gamma, \vec{\Gamma} \in \min ^{0} \vec{\partial}$ (см. определение III.1). Тогда справедливо неравенство

$$
\max _{\substack{\overrightarrow{\boldsymbol{\partial}}_{1} \in \gamma: \overrightarrow{\boldsymbol{\partial}} \in \gamma\left(\overrightarrow{\boldsymbol{\partial}}_{1}\right) \\ \min ^{0} \overrightarrow{\boldsymbol{\partial}} \subset \min ^{0} \overrightarrow{\boldsymbol{\partial}}_{1}}}\left|\sum_{\overrightarrow{\boldsymbol{\partial}}_{2} \in \min ^{0} \overrightarrow{\boldsymbol{\partial}}}(-1)^{\left|\overrightarrow{\boldsymbol{\partial}}_{2}\right|} W\left(V \mid \overrightarrow{\boldsymbol{\partial}}_{1} \backslash \overrightarrow{\boldsymbol{\partial}}_{2}\right)\right| \leqslant 2^{\left|\min ^{0} \overrightarrow{\boldsymbol{\partial}}\right|} h(\overrightarrow{\boldsymbol{\Gamma}}) \bar{W}(V),
$$

где $\left|\vec{\partial}_{2}\right|-$ число контуров в $\vec{\partial}_{2} u$

$$
\bar{W}(V)=\sum_{\substack{X: p_{0} X=V, \min _{\vec{i}} X=0}}^{0} h(X)\left|\varphi^{0}(X)\right|
$$

Также справедливо соответствующее неравенство для производных $W^{(k)}(\cdot)$, в котором в правой части стоит

$$
\bar{W}^{(k)}(V)=\sum_{\substack{X: p r X=V, \min _{\vec{i}} X=0}}^{0} h(X)\left|\left(\varphi^{0}(X)\right)^{(k)}\right|
$$

(здесь индекс $(k)$ означает некоторую частную производную по параметрам $\varepsilon, \mu)$. 
ДокАЗАтЕЛЬСтво. Учтем (26) и запишем сумму в левой части (27), выделяя пары, содержащие и не содержащие выделенный контур $\overrightarrow{\boldsymbol{\Gamma}}$, т.е. в виде

$$
\sum_{X}^{0} \varphi^{0}(X) \sum_{\overrightarrow{\boldsymbol{\partial}}_{3} \subset \min ^{0} \overrightarrow{\boldsymbol{\partial}} \backslash \overrightarrow{\boldsymbol{\Gamma}}}(-1)^{\left|\overrightarrow{\boldsymbol{\partial}}_{3}\right|}\left[h\left(X \mid \overrightarrow{\boldsymbol{\partial}}_{1} \cup \overrightarrow{\boldsymbol{\Gamma}} \backslash \overrightarrow{\boldsymbol{\partial}}_{3}\right)-h\left(X \mid \overrightarrow{\boldsymbol{\partial}}_{1} \backslash \overrightarrow{\boldsymbol{\partial}}_{3}\right)\right] .
$$

Из (25) имеем $h\left(X \mid \overrightarrow{\boldsymbol{\partial}}_{4}\right)-h\left(X \mid \overrightarrow{\boldsymbol{\partial}}_{4} \backslash \overrightarrow{\boldsymbol{\Gamma}}\right)=\sum_{k}\left[\Theta\left(X+k \vec{i} \mid \overrightarrow{\boldsymbol{\partial}}_{4}\right)-\Theta\left(X+k \vec{i} \mid \overrightarrow{\boldsymbol{\partial}}_{4} \backslash \overrightarrow{\boldsymbol{\Gamma}}\right)\right]$. Разность в квадратных скобках отлична от нуля, либо когда $X+k \vec{i}$ пересекает $\Gamma_{\infty}\left(\overrightarrow{\boldsymbol{\partial}}_{4}\right) \cup X_{1}\left(\overrightarrow{\boldsymbol{\partial}}_{4}\right)$ и не пересекает $\Gamma_{\infty}\left(\overrightarrow{\boldsymbol{\partial}}_{4} \backslash \overrightarrow{\boldsymbol{\Gamma}}\right) \cup X_{1}\left(\overrightarrow{\boldsymbol{\partial}}_{4} \backslash \overrightarrow{\boldsymbol{\Gamma}}\right)$, либо в противоположном случае. Если проекция $V_{\vec{\Gamma}}$ на ось $\vec{i}$ связна, то для всех ненулевых слагаемых контуры $X+k \vec{i}$ пересекают цилиндр с основанием $V(\overrightarrow{\boldsymbol{\Gamma}})-h(\overrightarrow{\boldsymbol{\Gamma}}) \vec{i}, V(\overrightarrow{\boldsymbol{\Gamma}}) \subset R_{0}^{\nu-1}$, высота его не более $2 h(\overrightarrow{\boldsymbol{\Gamma}})$, и, следовательно, квадратная скобка в $(30)$ не превосходит $2 h(\overrightarrow{\boldsymbol{\Gamma}})+$ $2 h(X)$. Если проекция $V_{\overrightarrow{\boldsymbol{\Gamma}}}$ на ось $\vec{i}$ несвязна, то необходимо вычислить сумму по связным компонентам $2 h_{j}(\overrightarrow{\boldsymbol{\Gamma}})+2 h_{j}(X)$; эта сумма не превосходит $2 h(\overrightarrow{\boldsymbol{\Gamma}}) h(X)$. Лемма доказана.

Несколько более сложно получается оценка величины $B(\overrightarrow{\boldsymbol{\Gamma}})($ см. (III.9) и (III.11)).

Лемма 5. Пусть $\vec{\partial} \in \gamma, \overrightarrow{\boldsymbol{\Gamma}} \in \min ^{0} \vec{\partial}, V(X):=\prod_{\Gamma \in X} V(\Gamma)$. Тогда справедливо неравенство

$$
B(\overrightarrow{\boldsymbol{\Gamma}}) \leqslant 2\left|H_{1}(\overrightarrow{\boldsymbol{\Gamma}})\right|+\left|H_{1}(\overrightarrow{\boldsymbol{\Gamma}} \mid \overrightarrow{\boldsymbol{\partial}} \backslash \overrightarrow{\boldsymbol{\Gamma}})\right| \leqslant r|\overrightarrow{\boldsymbol{\Gamma}}|_{1},
$$

где

$$
\begin{gathered}
H_{1}(\overrightarrow{\boldsymbol{\Gamma}} \mid \overrightarrow{\boldsymbol{\partial}})=H_{1}(\overrightarrow{\boldsymbol{\partial}})-H_{1}(\overrightarrow{\boldsymbol{\partial}} \backslash \overrightarrow{\boldsymbol{\Gamma}}), \quad r=C_{1} \sum_{x} \sum_{X: 0, x \in V(X)}^{0}\left|\varphi^{0}(X)\right|, \\
|\overrightarrow{\boldsymbol{\Gamma}}|_{1}=\left|\overrightarrow{\boldsymbol{\Gamma}}_{0}\right|_{1}+\sum_{\Gamma \in X_{1}}|\Gamma|, \quad \overrightarrow{\boldsymbol{\Gamma}}=\left(\overrightarrow{\boldsymbol{\Gamma}}_{0}, X_{1}\right),
\end{gathered}
$$

$\left|\overrightarrow{\boldsymbol{\Gamma}}_{0}\right|_{1},|\Gamma|-$ число $(\nu-1)$-мерных граней $\overrightarrow{\boldsymbol{\Gamma}}_{0}, \Gamma$, соответственно. Справедливы также неравенства на производные по $\varepsilon, \mu$, которые можно получить формальным дифберениированием (31).

ДоКАЗАТЕЛЬСтво. Из (21) и (22) находим, что

$$
H_{1}(\overrightarrow{\boldsymbol{\Gamma}})=\sum_{X}^{0} \Theta_{1}(X \mid \overrightarrow{\boldsymbol{\Gamma}}) \varphi^{0}(X), \quad H_{1}(\overrightarrow{\boldsymbol{\Gamma}} \mid \overrightarrow{\boldsymbol{\partial}})=\sum_{X}^{0} \varphi^{0}(X)\left[\Theta_{1}(X \mid \overrightarrow{\boldsymbol{\partial}})-\Theta_{1}(X \mid \overrightarrow{\boldsymbol{\partial}} \backslash \overrightarrow{\boldsymbol{\Gamma}})\right]
$$

где

$$
\Theta_{1}(X \mid \overrightarrow{\boldsymbol{\partial}})=\Theta(X \mid \overrightarrow{\boldsymbol{\partial}})-\sum_{q} \frac{\left|V_{q}(\overrightarrow{\boldsymbol{\partial}}) \cap \operatorname{pr} X\right|}{|\operatorname{pr} X|} \Theta(X \mid q) .
$$

Неравенства $\left|H_{1}(\overrightarrow{\boldsymbol{\Gamma}})\right| \leqslant r|\overrightarrow{\boldsymbol{\Gamma}}|_{1} / 6,\left|H_{1}(\overrightarrow{\boldsymbol{\Gamma}} \mid \overrightarrow{\boldsymbol{\partial}} \backslash \overrightarrow{\boldsymbol{\Gamma}})\right| \leqslant 2 r|\overrightarrow{\boldsymbol{\Gamma}}|_{1} / 3$ вытекают из следующих двух утверждений.

А. Если для каждого $X$ в $(32)$, для которого $\Theta_{1}(X \mid \overrightarrow{\boldsymbol{\Gamma}}) \neq 0$ и, следовательно, $\Theta_{1}(X \mid \overrightarrow{\boldsymbol{\partial}})-\Theta_{1}(X \mid \overrightarrow{\boldsymbol{\partial}} \backslash \overrightarrow{\boldsymbol{\Gamma}}) \neq 0$, найдется пара узлов $x, y \in X$ такая, что одна из ломаных, соединяющих $x$ и $y$ и состоящая из $\nu$ отрезков, параллельных осям координат, 
пересекает $V_{\vec{\Gamma}}$, тогда справедливо неравенство (31). Действительно, если воспользоваться в (32) рассуждениями из доказательства леммы 5.3.7 из [13] и неравенствами

$$
\left|\Theta_{1}(X \mid \overrightarrow{\boldsymbol{\Gamma}})\right| \leqslant 1, \quad\left|\Theta_{1}(X \mid \overrightarrow{\boldsymbol{\partial}})-\Theta_{1}(X \mid \overrightarrow{\boldsymbol{\partial}} \backslash \overrightarrow{\boldsymbol{\Gamma}})\right| \leqslant 2,
$$

получим утверждение леммы. Приступим к проверке условия утверждения "А". Заметим, что $\Theta_{1}(X \mid \overrightarrow{\boldsymbol{\Gamma}})=0$ (во втором соотношении $\Theta_{1}(X \mid \overrightarrow{\boldsymbol{\partial}})-\Theta_{1}(X \mid \overrightarrow{\boldsymbol{\partial}} \backslash \overrightarrow{\boldsymbol{\Gamma}})=0$ ), если найдется $i \in \bar{l}(\overrightarrow{\boldsymbol{\Gamma}})$, для которого $\operatorname{pr} X \subset V_{i}(\overrightarrow{\boldsymbol{\Gamma}})$. Ясно также, что условие утверждения "А" выполнены, если $X$ пересекает $V_{\overrightarrow{\boldsymbol{\Gamma}}}$. Таким образом, достаточно рассмотреть лишь $X$, не пересекающие $V_{\overrightarrow{\boldsymbol{\Gamma}}}$, для которых $\operatorname{pr} X$ не лежит целиком в некоторой связной компоненте $\mathbb{R}_{0}^{\nu-1} \backslash V_{\overrightarrow{\mathbf{\Gamma}}}$. Для каждого такого $X$ мы убедимся в справедливости второго утверждения.

Б. Существует $i \in \bar{l}(\overrightarrow{\boldsymbol{\Gamma}})$ такое, что

$$
A_{1}^{i}:=X \cap \Gamma_{\infty}^{k_{i}, 0} \cap \operatorname{pr}^{-1} V_{i}(\overrightarrow{\boldsymbol{\Gamma}}) \neq \varnothing, \quad A_{2}^{i}:=X \cap\left(\Gamma_{\infty}^{k_{i}, 0} \backslash \operatorname{pr}^{-1} V_{i}(\overrightarrow{\boldsymbol{\Gamma}})\right) \neq \varnothing .
$$

Заметим, что из $A_{1}^{i} \neq \varnothing$ вытекает $A_{2}^{i} \neq \varnothing$, так как иначе $\operatorname{pr} X$ целиком лежит в некоторой связной компоненте $R_{0}^{\nu-1} \backslash V_{\overrightarrow{\boldsymbol{\Gamma}}}$ или пересекает $V_{\overrightarrow{\boldsymbol{\Gamma}}}$. Убедимся, что из "Б" вытекает "А". Пусть $x \in A_{1}^{i}, y \in A_{2}^{i}$. Обозначим $y(x)$ проекцию $y$ на плоскость $\Gamma_{\infty}^{k_{i}, 0}$. Тогда ломаная, которая соединяет $x$ и $y(x)$, пересекает край $\overrightarrow{\boldsymbol{\Gamma}}_{0}$; соединив $y(x)$ и $y$, получаем ломаную, пересекающую $\overrightarrow{\boldsymbol{\Gamma}}_{0}$. Итак, нам осталось доказать "Б". Пусть $\Theta(X \mid \overrightarrow{\boldsymbol{\Gamma}})=0$, тогда $\left|V_{i}(\overrightarrow{\boldsymbol{\Gamma}}) \cap \operatorname{pr} X\right| \Theta\left(X \mid k_{i}\right) \neq 0$ для некоторого $i \in \bar{l}(\overrightarrow{\boldsymbol{\Gamma}})$, отсюда $A_{1}^{i} \neq \varnothing$. Для доказательства "Б" при $\Theta_{1}(X \mid \overrightarrow{\boldsymbol{\partial}})-\Theta_{1}(X \mid \overrightarrow{\boldsymbol{\partial}} \backslash \overrightarrow{\boldsymbol{\Gamma}}) \neq 0$ представим эту разность в виде

$$
\begin{aligned}
\Theta_{1}(X \mid \overrightarrow{\boldsymbol{\partial}}) & -\Theta_{1}(X \mid \overrightarrow{\boldsymbol{\partial}} \backslash \overrightarrow{\boldsymbol{\Gamma}})=\Theta(X \mid \overrightarrow{\boldsymbol{\partial}})-\Theta(X \mid \overrightarrow{\boldsymbol{\partial}} \backslash \overrightarrow{\boldsymbol{\Gamma}})+ \\
& +\frac{|V(\overrightarrow{\boldsymbol{\Gamma}}) \cap \operatorname{pr} X|}{|\operatorname{pr} X|} \Theta(X \mid q)-\sum_{i \in l(\overrightarrow{\boldsymbol{\Gamma}})} \frac{\left|V_{i}(\overrightarrow{\boldsymbol{\Gamma}}) \cap \operatorname{pr} X\right|}{|\operatorname{pr} X|} \Theta\left(X \mid k_{i}\right),
\end{aligned}
$$

где $q$ - внешняя фаза контура $\overrightarrow{\boldsymbol{\Gamma}}, l(\overrightarrow{\boldsymbol{\Gamma}})$ - номера конечных связных компонент $R^{\nu} \backslash V_{\overrightarrow{\boldsymbol{\Gamma}}}$. Из этого представления, а также из определения (33) вытекают неравенства (34). Пусть $\Theta(X \mid \overrightarrow{\boldsymbol{\partial}})=\Theta(X \mid \overrightarrow{\boldsymbol{\partial}} \backslash \overrightarrow{\boldsymbol{\Gamma}})$, т.е. обе эти функции принимают одновременно значения 1 или 0. Тогда существует $i$ такое, что $\Theta\left(X \mid k_{i}\right)\left|\operatorname{pr} X \cap V_{i}(\overrightarrow{\boldsymbol{\Gamma}})\right| \neq 0$ и тем самым $A_{1}^{i} \neq \varnothing$. Пусть теперь $\Theta(X \mid \overrightarrow{\boldsymbol{\partial}})=1, \Theta(X \mid \overrightarrow{\boldsymbol{\partial}} \backslash \overrightarrow{\boldsymbol{\Gamma}})=0$. Тогда множество $X \cap \mathrm{pr}^{-1} V(\overrightarrow{\boldsymbol{\Gamma}})$ непусто, следовательно, $A_{1}^{i} \neq \varnothing$. Рассмотрим последний случай, когда $\Theta(X \mid \overrightarrow{\boldsymbol{\partial}})=0$, $\Theta(X \mid \overrightarrow{\boldsymbol{\partial}} \backslash \overrightarrow{\boldsymbol{\Gamma}})=1$. Если pr $X \subset V(\overrightarrow{\boldsymbol{\Gamma}})$, то имеем $|\operatorname{pr} X \cap V(\overrightarrow{\boldsymbol{\Gamma}})| \Theta(X \mid q) /|\operatorname{pr} X|=1$ и $\left|\operatorname{pr} X \cap V_{i}(\overrightarrow{\boldsymbol{\Gamma}})\right| \Theta\left(X \mid k_{i}\right) \neq 0$ для некоторого $i$, откуда $A_{1}^{i} \neq \varnothing$. Если же $\operatorname{pr} X \not \subset V(\overrightarrow{\boldsymbol{\Gamma}})$, то для внешней компоненты $i$ множество $A_{1}^{i} \neq \varnothing$, что и завершает доказательство леммы.

В следующей лемме приведены более явные оценки величин $r$ и

$$
\bar{W}_{2 x}(\delta, \varepsilon, \mu)=\sum_{x \in \mathbb{Z}_{0}^{\nu-1}}|x| \sum_{V \subset \mathbb{Z}_{0}^{\nu-1}: 0, x \in V}(1+\delta)^{|V|-1} \bar{W}(V) .
$$


Лемма 6. Существуют константы $\varepsilon_{0}, C_{i}$, зависящие лищь от $\nu$, такие, что npu

$$
\operatorname{Re} \varepsilon \geqslant \varepsilon_{0}, \quad\left|e^{-2 \mu}\right|+C_{2} \psi^{2 \nu}\left|1-e^{-2 \mu}\right| \leqslant 1,
$$

где $\psi=e^{-\operatorname{Re} \varepsilon}$, выполнены неравенства

$$
\begin{gathered}
r \leqslant C_{3} \psi^{2 \nu}, \quad r_{\varepsilon}^{\prime} \leqslant C_{4} \psi^{2 \nu}, \quad r_{\mu}^{\prime} \leqslant C_{5} \psi^{2 \nu}, \\
\bar{W}_{2 x}(1, \varepsilon, \mu) \leqslant C_{6} \psi^{2 \nu}, \quad \bar{W}_{2 x}(1, \varepsilon, \mu)_{\varepsilon}^{\prime} \leqslant C_{7} \psi^{2 \nu}, \quad \bar{W}_{2 x}(1, \varepsilon, \mu)_{\mu}^{\prime} \leqslant C_{8} \psi^{2 \nu} ;
\end{gathered}
$$

здесъ $r_{\varepsilon}^{\prime}$ и другие производные определены так же, как в формуле (29).

Лемма 6 вытекает из лемм $2,3,5$, а также из неравенств $|x| 2^{|\operatorname{pr} X|} \leqslant \exp \sum_{\Gamma \in X}|\Gamma|$ при $0, x \in \operatorname{pr} X,|V(\Gamma)| \leqslant(|\Gamma| /(2 \nu))^{\nu /(\nu-1)}$.

Таким образом, леммы 4, 6 доказывают, что выполнено условие 3 теоремы III.1. Леммы 5, 6 и следующая лемма 7 покажут, что выполнено условие 4 этой теоремы.

Лемма 7. Существуют константъ $C_{9}, C_{10}$, зависящие лищь от $\nu$, такие, что в области

$$
\operatorname{Re} \varepsilon \geqslant \varepsilon_{0}, \quad\left|e^{-2 \mu}\right|+C_{9} \psi^{2 \nu / 3}\left|1-e^{-2 \mu}\right| \leqslant 1
$$

справедливо неравенство

$$
\sum_{\substack{\overrightarrow{\boldsymbol{\Gamma}}: \min _{1} V_{q}^{\prime}(\overrightarrow{\boldsymbol{\Gamma}})=0,|\overrightarrow{\boldsymbol{\Gamma}}|=\text { const }}}\left|\exp \left\{-H_{0}(\overrightarrow{\boldsymbol{\Gamma}})-\max _{q} h_{q}\left|V_{\overrightarrow{\boldsymbol{\Gamma}}}\right|+\Delta \vec{h} m_{1}(\overrightarrow{\boldsymbol{\Gamma}})+B_{1}(\overrightarrow{\boldsymbol{\Gamma}})\right\}\right| \leqslant\left(C_{10} \psi\right)^{|\overrightarrow{\boldsymbol{\Gamma}}| / 2},
$$

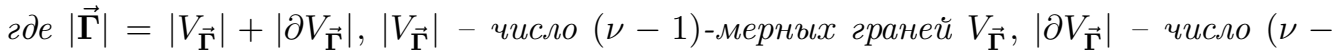
2)-мерных граней $V_{\vec{\Gamma}}$, не лежащих в $(\nu-1)$-мерных гранях $V_{\vec{\Gamma}}$.

ДокАЗАТЕЛЬСтво. Если в правой части (39) суммировать по контурам вида $\left(\overrightarrow{\boldsymbol{\Gamma}}_{0}, \varnothing\right)$, то из формулы $(20)$ и неравенства $(16)$ в этом случае имеем $e^{-H_{0}(\overrightarrow{\boldsymbol{\Gamma}})}=$ $e^{-\varepsilon\left(\left|\overrightarrow{\boldsymbol{\Gamma}}_{0}\right|_{1}-\left|V_{\overrightarrow{\boldsymbol{\Gamma}}_{0}}\right|\right)} \leqslant e^{-2 \varepsilon\left(\left|\overrightarrow{\boldsymbol{\Gamma}}_{0}\right|_{1}\right) / 3}$, откуда получаем $(39)$. Если в (39) суммировать по контурам вида $\left(q, X_{1}\right)\left(\operatorname{pr} X_{1}\right.$ связна), то с учетом явного вида $e^{-H_{0}(\overrightarrow{\boldsymbol{\Gamma}})}($ см. $(20))$ можно оценить $F^{X_{1}}$ следующим образом:

$$
\left|F^{X_{1}}\right| \leqslant\left(e^{-\varepsilon|\Gamma| / 2}\right)^{X_{1}} e^{-\varepsilon\left|\operatorname{pr} X_{1}\right| / 2} .
$$

При суммировании по $X_{1}$ при фиксированной проекции $\operatorname{pr} X_{1}$ первый сомножитель в правой части (40) является константой, а оценка второго сомножителя дает то же выражение, что и оценка $\sigma\left(\mathrm{pr}^{-1} X_{1}\right)$, см. (8), но теперь энергия на единицу площади контура в два раза меньше. Тем не менее мы можем воспользоваться леммой III.4, оценив эту сумму $\left(\mathrm{pr}^{-1} X_{1}\right.$ фиксирована) единицей. Таким образом, если суммировать в правой части (39) лишь по контурам вида $\left(q, X_{1}\right)$, оценка $(39)$ вытекает из следующего утверждения: число связных $V \subset \mathbb{Z}_{0}^{\nu-1}$, для которых $|V|=$ const, $0 \in V$, не превосходит $(2 \nu)^{2|V|}$. В общем случае у контура $\overrightarrow{\boldsymbol{\Gamma}}=\left(\overrightarrow{\boldsymbol{\Gamma}}_{0}, X_{1}\right)$ носитель представляет собой $V_{\overrightarrow{\boldsymbol{\Gamma}}_{0}} \cup \operatorname{pr} X_{1}$ и является связным множеством, а сами $V_{\overrightarrow{\boldsymbol{\Gamma}}_{0}}, \operatorname{pr} X_{1}$ состоят из нескольких связных компонент. Доказательство неравенства (39) в этой общей ситуации вытекает из рассмотренных случаев. 
Заметим, что неравенство (39) остается верным при замене $e^{-H_{0}(\overrightarrow{\boldsymbol{\Gamma}})}$ на $\partial e^{-H_{0}(\overrightarrow{\boldsymbol{\Gamma}})} / \partial \varepsilon$ с большей, чем $C_{10}$, константой. При замене $e^{-H_{0}(\overrightarrow{\boldsymbol{\Gamma}})}$ на $\partial e^{-H_{0}(\overrightarrow{\boldsymbol{\Gamma}})} / \partial \mu$ в правую часть (39) необходимо добавить сомножитель $\max _{q}\left(q e^{-q \mu}\right) \leqslant 1 / \mu$. Из лемм 4-7 и теоремы III.1 вытекает следующая теорема.

Теорема 1. Существует константа $\varepsilon_{0}$, зависящая лищь от $\nu$, такая, что область

$$
\mathcal{M}=\left\{(\varepsilon, \mu): \varepsilon>\varepsilon_{0}, \mu>0\right\}
$$

является областъю фазовых переходов первого рода относительно введенных фаз модели Изинга в $\mathbb{Z}_{+}^{\nu}$ с неустойчивым граничным условием на подложке. $B$ замыкании $\overline{\mathcal{M}}$ области $\mathcal{M}$ величины $\bar{\omega}, \bar{\omega}_{p}^{\prime}, \bar{\omega}_{\varepsilon}^{\prime}$ (см. теоремы I.4-I.7) не превосходят $C e^{-2 \varepsilon(\nu-1)}$, в $\mathcal{M}$ справедливо неравенство $\bar{\omega}_{\mu}^{\prime}<C e^{-2 \varepsilon(\nu-1)} / \mu$.

В частности, справедливы следующие утверждения. Решения уравнения состояния "поверхностных" фаз и соответствующих контурных уравнений

$$
\begin{gathered}
\overrightarrow{\mathbf{p}}=\overrightarrow{\mathbf{h}}+\overrightarrow{\mathbf{s}}(\overrightarrow{\mathbf{p}}, \varepsilon, \mu), \\
\overrightarrow{\mathcal{F}}=\overrightarrow{\mathbf{\Upsilon}}\left(\overrightarrow{\mathcal{F}}, \Delta \mathbf{p}, e^{\left.-p_{\max } V_{\vec{\partial}} \varphi_{\overrightarrow{\boldsymbol{\partial}}}\right)}\right.
\end{gathered}
$$

существуют, непрерывны по $\overrightarrow{\mathbf{h}}$ и слабо непрерывны по $\varepsilon, \mu n p u \mathbf{\mathbf { h }} \in \mathbb{R}^{\mathbb{Z}_{+}}\left(h_{q}-\right.$ ограниченная сверху последовательность) $u(\varepsilon, \mu) \in \overline{\mathcal{M}} . \Pi$ При $(\varepsilon, \mu) \in \mathcal{M} u \overrightarrow{\mathbf{h}}=$ $\overrightarrow{\mathbf{h}}(\varepsilon, \mu)$ уравнение состояния определяет поверхностную свободную энергию

$$
-p(\varepsilon, \mu)=\min _{q}\left(-p_{q}(\varepsilon, \mu)\right)
$$

совпадающую с термодинамическим пределом (4), который при вещественных параметрах не зависит от $q$. Поверхностная свободная энергия является вещественно-аналитической функцией внутри каждого страта фазовой диаграммы

$$
G(\varepsilon, \mu)=\left\{q \in \mathbb{Z}_{+}: \min _{q_{1}}\left(-p_{q_{1}}(\varepsilon, \mu)\right)=-p_{q}(\varepsilon, \mu)\right\}
$$

Производные $\partial p / \partial \varepsilon, \partial p / \partial \mu$ продолжаются до непрерывных функций в замыкании стратов $\partial_{q} \mathcal{M}=\{(\varepsilon, \mu) \in \mathcal{M}: G(\varepsilon, \mu)=q\} u$

$$
\begin{aligned}
\frac{\partial p}{\partial \mu} & =\frac{1}{2} \lim _{\substack{N \rightarrow \infty \\
\Lambda_{S} \uparrow \mathbb{Z}_{0}^{\nu-1}}} \frac{1}{\left|\Lambda_{S}\right|} \sum_{x \in \Lambda}\left(\left\langle\sigma_{x}\right\rangle_{q, \Lambda}-\left\langle\sigma_{x}\right\rangle_{+, \Lambda}\right), \\
\frac{\partial p}{\partial \varepsilon} & =\lim _{\substack{N \rightarrow \infty, \Lambda_{S} \uparrow \mathbb{Z}_{0}^{\nu-1}}} \frac{1}{\left|\Lambda_{S}\right|} \sum_{[x, y] \cap \Lambda \neq \varnothing}\left(\left\langle\sigma_{x} \sigma_{y}\right\rangle_{q, \Lambda}-\left\langle\sigma_{x} \sigma_{y}\right\rangle_{+, \Lambda}\right),
\end{aligned}
$$

где $\langle\cdot\rangle_{q, \Lambda},\langle\cdot\rangle_{+, \Lambda}-$ средние значения по ансамблю со статистическими суммами $\Xi\left(\Lambda \mid \sigma^{q}\right), \Xi\left(\Lambda \mid \sigma^{+}\right)$, соответственно. 


\section{4. ДОПОЛНИТЕЛЬНЫЕ ОЦЕНКИ НА ЛИНИЯХ СОСУЩЕСТВОВАНИЯ ФАЗ}

В этом разделе доказаны дополнительные оценки, позволяющие убедиться в разрывности производных поверхностной свободной энергии $-p(\varepsilon, \mu)$ и найти оценки функций, которые задают линии сосуществования поверхностных фаз.

ЛЕмма 8. Существуют константы $\varepsilon_{0}, C_{i}$, зависящие лишь от $\nu$, такие, что в области $\mathcal{M}$ справедливы неравенства

$$
\begin{aligned}
& \left|s_{q}-s_{q+1}\right| \leqslant C_{1} \mu e^{-2 \varepsilon}+e^{\left(C_{2}-\varepsilon_{1}\right)(q+1)}, \\
& \left|p_{q}-p_{q+1}\right| \leqslant \mu\left(C_{3} e^{-2 \varepsilon}+1\right)+e^{\left(C_{2}-\varepsilon_{1}\right)(q+1)},
\end{aligned}
$$

где $\varepsilon_{1}:=2(\nu-1) \varepsilon, \varepsilon \geqslant \varepsilon_{0}$.

ДокАзАтЕЛьство. Каждой границе $\vec{\partial} \in \gamma_{q}$ сопоставим границу $T \vec{\partial} \in \gamma_{q+1}$, которая получается сдвигом границы $\vec{\partial}$ на единицу вверх. Определим функции

$$
\begin{aligned}
& \varphi_{\vec{\partial}}^{q}:=\left\{\begin{array}{lll}
0, & \text { если } & \vec{\partial} \in \gamma_{q+1} \backslash T \gamma_{q}, \\
\varphi_{T^{-1}} \vec{\partial}, & \text { если } & \vec{\partial} \notin \gamma_{q+1} \backslash T \gamma_{q},
\end{array}\right. \\
& \varphi_{\vec{\partial}}^{\Theta}:=\Theta \varphi_{\vec{\partial}}^{q}+(1-\Theta) \varphi_{\vec{\partial}},
\end{aligned}
$$

где $\varphi_{\vec{\partial}}$ определено гамильтонианом $H(\overrightarrow{\boldsymbol{\partial}})($ см. (I.8)). Положим для $0 \leqslant \Theta \leqslant 1$

$$
\Delta p_{q+1}^{\Theta}:=\Theta \Delta p_{q}+(1-\Theta) \Delta p_{q+1}, \quad q \geqslant 0 ; \quad \Delta p_{0}^{\Theta}:=\Delta p_{0} .
$$

Рассмотрим контурные уравнения (43) на $\mathcal{F}^{\Theta}\left(\partial^{q+1}\right)$, в которых $\varphi_{\vec{\partial}}$ заменено на $\varphi_{\vec{\partial}}^{\Theta}$ и $\Delta p_{q}-$ на $\Delta p_{q}^{\Theta}: \mathcal{F}^{\Theta}\left(\partial^{q+1}\right)=\Upsilon\left(\partial^{q+1} ; \overrightarrow{\mathcal{F}}^{\Theta}, \Delta \overrightarrow{\mathbf{p}}^{\Theta}, e^{-p_{\max } V_{\vec{\partial}}} \varphi_{\vec{\partial}}^{\Theta}\right), q \geqslant 0 ; \mathcal{F}^{\Theta}\left(\partial^{0}\right):=\mathcal{F}\left(\partial^{0}\right)$.

Из определений вытекает, что $\left.\mathcal{F}^{\Theta}\left(\partial^{q+1}\right)\right|_{\Theta=1}=\mathcal{F}\left(\partial^{q+1}\right)$ и $\left.\mathcal{F}^{\Theta}\left(\partial^{q+1}\right)\right|_{\Theta=0}=\mathcal{F}\left(\partial^{q}\right)$. Таким образом, для оценки разностей $\mathcal{F}\left(\partial^{q+1}\right)-\mathcal{F}\left(\partial^{q}\right)$ и $s_{q+1}-s_{q}$ достаточно найти не зависящую от $\Theta$ оценку производных $\partial \mathcal{F}^{\Theta} / \partial \Theta, \partial s_{q}^{\Theta} / \partial \Theta$. Используя теорему III.5, выберем величину $\bar{\omega}_{q}^{\prime}$ в виде

$$
\omega_{q}^{\prime}:=C_{4} \mu e^{-2 \varepsilon}+e^{\left(C_{5}-\varepsilon_{1}\right)(q+1)},
$$

указав параметр дифференцирования, и убедимся, что найдутся константы $C_{i}, \varepsilon_{0}$, для которых при $\varepsilon>\varepsilon_{0}$ выполнено неравенство (III.82), если справедливо следующее неравенство на $p_{q}$ :

$$
\left|p_{q+1}-p_{q}-h_{q}+h_{q+1}\right| \leqslant C_{1} \mu e^{-2 \varepsilon}+e^{\left(C_{2}-\varepsilon_{1}(q+1)\right)} .
$$

Предварительно докажем неравенство

$$
\sum_{\substack{\overrightarrow{\boldsymbol{\partial}}: \overrightarrow{\boldsymbol{\partial}}^{\text {ex }}=\partial^{q} \stackrel{*}{\cap} 0 \neq \varnothing, V_{k}(\overrightarrow{\boldsymbol{\partial}}) \neq \varnothing}}|V(\overrightarrow{\boldsymbol{\partial}})|^{l+1} \mid \varphi_{\overrightarrow{\boldsymbol{\partial}}} e^{\bar{\omega} \overrightarrow{\boldsymbol{\partial}}-p V_{\vec{\partial}} \bar{\sigma}(\overrightarrow{\boldsymbol{\partial}}) \bar{\sigma}_{q}\left(V\left(\partial^{q}\right)\right) \mid \leqslant e^{\left(C_{l}-\varepsilon_{1}\right)|q-k|},},
$$


где $C_{l}$ зависит лишь от $l, \nu$. Здесь условие суммирования $\partial \stackrel{*}{\cap} 0 \neq \varnothing$ означает следующее:

$$
\begin{aligned}
\sum_{\partial: \partial \cap 0 \neq \varnothing} \bar{\omega}(\partial):= & \sum_{\Gamma: \min _{1} \Gamma=0}\left\{C_{1}|\Gamma| \sum_{\partial \ni \Gamma} \bar{\omega}(\partial)+\right. \\
& \left.+C_{2} \sum_{\substack{\partial: \Gamma_{0} \subset V(\Gamma), \partial \ni \Gamma}} \bar{\omega}(\partial)+C_{3} \sum_{\Gamma_{1}, \partial: \partial \ni \Gamma, \Gamma_{1}} d\left(\Gamma, \Gamma_{1}\right) \bar{\omega}(\partial)\right\} .
\end{aligned}
$$

Очевидно, что для каждой границы $\overrightarrow{\boldsymbol{\partial}}=\left(\overrightarrow{\boldsymbol{\partial}}_{0}, X_{1}\right)$ число вертикальных граней в $\overrightarrow{\boldsymbol{\partial}}_{0}$ не меньше $2(\nu-1)|q-k|$, т.е. $\left|\overrightarrow{\boldsymbol{\partial}}_{0}\right|_{1} \geqslant 2(\nu-1)|q-k|$. Теорема III.3 дает мажорантную оценку левой части $(50)$ относительно $e^{-H_{0}(\overrightarrow{\boldsymbol{\Gamma}})}$, следовательно, из леммы 3 вытекает, что эта оценка мажорантна относительно $e^{-\varepsilon|\overrightarrow{\boldsymbol{\partial}}|_{1, \text { vert }}}$. Учитывая, что суммы в левой части (50) сходятся (см. замечание об оценках в разделе III.2), получаем (50). При помощи леммы 3 получаем также, что

$$
\left|h_{q}^{1}-h_{q+1}^{1}\right| \leqslant e^{\left(C_{2}-\varepsilon_{1}\right)(q+1)-2 \varepsilon} .
$$

Возвращаясь к рассмотрению неравенства (III.82), из неравенств (49), (50) находим, что в левой части (III.82) слагаемые, содержащие $\bar{\omega}_{k_{i}}^{\prime}, \Delta p_{k_{i}}^{\Theta^{\prime}}$, не превосходят величины $\sum_{k \neq q, k>0} e^{\left(C-\varepsilon_{1}\right)|q-k|}\left(\bar{\omega}_{k}^{(1), \theta}+\mu+\bar{\omega}_{k}^{(1), \theta}\right)$. Рассмотрим в левой части (III.82) слагаемые, содержащие $\varphi_{\vec{\partial}}^{\Theta^{\prime}}=\varphi_{\vec{\partial}}^{q}-\varphi_{\vec{\partial}}$. Пусть $\varphi_{\vec{\partial}}^{q}=0$, т.е. $\vec{\partial} \in \gamma_{q+1} \backslash T \gamma_{q}$. В этом случае либо а) $\left|\vec{\partial}_{0}\right|_{1} \geqslant 2(\nu-1)|q-k|$, либо б) $X_{1} \cap \mathbb{Z}_{0}^{\nu-1} \neq \varnothing$. Из (50) в случае "а" получаем, что слагаемое, содержащее $\varphi_{\vec{\partial}}^{\Theta^{\prime}}$, можно оценить величиной $e^{\left(C_{2}-\varepsilon_{1}\right)(q+1)}$. Слагаемые, для которых верно “б”, содержат множитель $1-e^{-\mu} \leqslant \mu$ (см. (14) и (8)), а оставшуюся часть этого слагаемого можно оценить величиной $C e^{-\varepsilon_{1}}$.

Таким образом, в левой части (III.82) нам осталось оценить слагаемые, для котоpых $\vec{\partial} \notin \gamma_{q+1} \backslash T \gamma_{q}$ или $T^{-1} \overrightarrow{\boldsymbol{\partial}} \in \gamma_{q}$. В этом случае $\varphi_{\vec{\partial}}^{\Theta^{\prime}}=\varphi_{T^{-1}} \overrightarrow{\boldsymbol{\partial}}-\varphi_{\vec{\partial}}$. Запишем $\varphi_{\vec{\partial}}$ в виде $z(\overrightarrow{\boldsymbol{\Gamma}})^{\vec{\partial}} \varphi([\overrightarrow{\boldsymbol{\partial}}])$, выделив в виде сомножителя из функций Урселла активности $z(\overrightarrow{\boldsymbol{\Gamma}}):=\exp \left\{-H_{0}(\overrightarrow{\boldsymbol{\Gamma}})\right\} ; \varphi([\overrightarrow{\boldsymbol{\partial}}])$ зависит лишь от $W(V \mid \overrightarrow{\boldsymbol{\partial}})$. Введем для $0 \leqslant \Theta \leqslant 1$

$$
\begin{gathered}
z_{\Theta_{1}}(\overrightarrow{\boldsymbol{\Gamma}}):=\Theta_{1} z\left(T^{-1} \overrightarrow{\boldsymbol{\Gamma}}\right)+\left(1-\Theta_{1}\right) z(\overrightarrow{\boldsymbol{\Gamma}}), \\
W\left(V \mid \overrightarrow{\boldsymbol{\partial}} ; \Theta_{1}\right):=\Theta_{1} W\left(V \mid T^{-1} \overrightarrow{\boldsymbol{\partial}}\right)+\left(1-\Theta_{1}\right) W(V \mid \overrightarrow{\boldsymbol{\partial}}) \\
\varphi_{\overrightarrow{\boldsymbol{\partial}}}\left(\Theta_{1}\right):=\left\{z_{\Theta_{1}}(\overrightarrow{\boldsymbol{\Gamma}})\right\}^{\overrightarrow{\boldsymbol{\partial}}} \varphi\left([\overrightarrow{\boldsymbol{\partial}}] ; W\left(V \mid \overrightarrow{\boldsymbol{\partial}} ; \Theta_{1}\right)\right):=z_{\Theta_{1}}(\overrightarrow{\boldsymbol{\Gamma}})^{\vec{\partial}} \varphi_{\Theta_{1}}([\overrightarrow{\boldsymbol{\partial}}]) .
\end{gathered}
$$

По определению $\varphi_{\Theta_{1}=0, \vec{\partial}}=\varphi_{\vec{\partial}}$ и $\varphi_{\Theta_{1}=1, \vec{\partial}}=\varphi_{T^{-1} \vec{\partial}}$. Разность $\varphi_{T^{-1} \overrightarrow{\boldsymbol{\partial}}}-\varphi_{\overrightarrow{\boldsymbol{\partial}}}$. совпадает для некоторого $\Theta_{1}$ с $\partial \varphi_{\vec{\partial}}\left(\Theta_{1}\right) / \partial \Theta_{1}$ и равна

$$
\sum_{\overrightarrow{\boldsymbol{\Gamma}} \in \overrightarrow{\boldsymbol{\partial}}}\left(z\left(T^{-1} \overrightarrow{\boldsymbol{\Gamma}}\right)-z(\overrightarrow{\boldsymbol{\Gamma}})\right) z_{\Theta_{1}}(\overrightarrow{\boldsymbol{\Gamma}}) \overrightarrow{\boldsymbol{\partial}} \backslash \overrightarrow{\boldsymbol{\Gamma}} \varphi_{\Theta_{1}}(\overrightarrow{\boldsymbol{\partial}})+\sum_{V}\left(W\left(V \mid T^{-1} \overrightarrow{\boldsymbol{\partial}}\right)-W(V \mid \overrightarrow{\boldsymbol{\partial}})\right) \frac{\partial \varphi_{\Theta_{1}}([\overrightarrow{\boldsymbol{\partial}}])}{\partial W\left(V \mid \overrightarrow{\boldsymbol{\partial}} ; \Theta_{1}\right)}
$$


При этом $\left|z\left(T^{-1} \overrightarrow{\boldsymbol{\Gamma}}\right)-z(\overrightarrow{\boldsymbol{\Gamma}}):=z\left(T^{-1} \overrightarrow{\boldsymbol{\Gamma}}\right)\left(e^{-\mu V_{\overrightarrow{\boldsymbol{\Gamma}}}}-1\right)\right| \leqslant\left|z\left(T^{-1} \overrightarrow{\boldsymbol{\Gamma}}\right)\right| \mu \mid V_{\overrightarrow{\boldsymbol{\Gamma}}}$, что дает после суммирования оценку $C \mu e^{-\varepsilon_{1}}$. Из определения (21) получаем, что

$$
\left|W\left(V \mid T^{-1} \overrightarrow{\boldsymbol{\partial}}\right)-W(V \mid \overrightarrow{\boldsymbol{\partial}})\right| \leqslant \sum_{\substack{X: \operatorname{pr} X=V, h(X) \geqslant k_{\operatorname{ex}}\left(\overrightarrow{\boldsymbol{\partial}}_{1}(V)\right)=k}}^{0}\left|\varphi^{0}(X)\right|,
$$

где $\overrightarrow{\boldsymbol{\partial}}_{1}(V)$ - минимальная подграница в $\overrightarrow{\boldsymbol{\partial}}$, для которой носитель каждого контуpa $\overrightarrow{\boldsymbol{\Gamma}} \in \min ^{0} \overrightarrow{\boldsymbol{\partial}}_{1}(V)$ пересекает $V$. Для каждого $X$ левая часть последнего неравенства содержит, очевидно, $e^{-\varepsilon_{1}(k+1)-2 \varepsilon}$. Если использовать теорему III.3, лемму III.5 и неравенство (49), получаем, что соответствующее слагаемое в (III.82) оценивается величиной $e^{\left(C-\varepsilon_{1}\right)(k+1)-2 \varepsilon}$. Суммируя полученные неравенства, получаем, что (III.82) выполнено (величина $\bar{\omega}_{q}^{\prime}$ определяется в (48)) при условии, что компоненты вектора $\overrightarrow{\mathbf{p}}$ удовлетворяют неравенству (49) и, следовательно,

$$
\left|s_{q}(\overrightarrow{\mathbf{p}}, \varepsilon, \mu)-s_{q+1}(\overrightarrow{\mathbf{p}}, \varepsilon, \mu)\right| \leqslant \bar{\omega}_{q}^{(1), \theta} .
$$

Чтобы доказать (46), (47), где $\overrightarrow{\mathbf{p}}=\overrightarrow{\mathbf{p}}(\varepsilon, \mu)$ - решение уравнения состояния (41), обозначим $\overrightarrow{\mathbf{p}}_{(k)} \quad k$-ю итерацию уравнения состояния, т.е. положим $\overrightarrow{\mathbf{p}}_{(k)}=\overrightarrow{\mathbf{h}}+$ $\overrightarrow{\mathbf{s}}\left(\overrightarrow{\mathbf{p}}_{(k-1)}, \varepsilon, \mu\right), \overrightarrow{\mathbf{p}}_{(0)}=\overrightarrow{\mathbf{h}}$. Ясно, что для $\overrightarrow{\mathbf{p}}_{(0)}$ неравенство (49) выполнено, следовательно, для него выполнено и неравенство (52). Предположим, что неравенство (49) выполнено для $\overrightarrow{\mathbf{p}}_{(k-1)}$. Из неравенства (52) и определения $\overrightarrow{\mathbf{p}}_{(k)}$ вытекает, что (49) выполнено и для $\overrightarrow{\mathbf{p}}_{(k)}$ и, следовательно, для предельного значения $\overrightarrow{\mathbf{p}}(\varepsilon, \mu)$. Таким образом, лемма 8 доказана.

ЛЕмма 9. Если $\{q, q+1\} \subset G(\varepsilon, \mu)$, то при $\varepsilon \geqslant \varepsilon_{0}$, где $\varepsilon_{0}$ достаточно велико, справедливо неравенство

$$
\left|\mu-e^{\left(-\varepsilon_{1}(q+1)\right)}\right| \leqslant e^{\left(C-\varepsilon_{1}\right)(q+1)-2 \varepsilon},
$$

где $C$ - некоторая константа.

Убедимся, что (53) есть следствие неравенства

$$
\left|s_{q}-s_{q+1}+e^{-\varepsilon_{1}(q+1)}\right| \leqslant \mu C_{1} e^{-2 \varepsilon}+e^{\left(C-\varepsilon_{1}\right)(q+1)-2 \varepsilon} .
$$

Действительно, если $\{q, q+1\} \subset G(\varepsilon, \mu)$, то $0=p_{q+1}-p_{q}=\mu+h_{q}^{1}-h_{q+1}^{1}+s_{q}-s_{q+1}$. Отсюда $\left|\mu-e^{\left(-\varepsilon_{1}(q+1)\right)}\right| \leqslant\left|h_{q}^{1}-h_{q+1}^{1}\right|+\left|s_{q}-s_{q+1}+e^{\left(-\varepsilon_{1}(q+1)\right)}\right|$. Учитывая, что из леммы 8 вытекает неравенство $|\mu| \leqslant e^{\left(C-\varepsilon_{1}\right)(q+1)}$, получаем из предыдущего неравенства и неравенства (54) искомое неравенство (53). Доказательство неравенства (54) является небольшой модификацией доказательства леммы 8. При этом дополнительно используется то, что низшая степень разложения по $e^{-\varepsilon}$ в сумме $s_{q}-s_{q+1}+e^{-\varepsilon_{1}(q+1)}$ равна $2(\nu-1) q+2$. Напомним, что $s_{q}$ является рядом по степенями $\varphi_{\vec{\partial}}$ и в $s_{q+1}$ выражение $e^{-\varepsilon_{1}(q+1)}$ содержится в $\varphi_{\vec{\partial}^{0}}$, где $\overrightarrow{\boldsymbol{\partial}}^{0}=\left(\overrightarrow{\boldsymbol{\partial}}_{0}, \varnothing\right),\left|\overrightarrow{\boldsymbol{\partial}}_{0}\right|_{1}=2(\nu-1)(q+1)$, $\left|V\left(\vec{\partial}_{0}\right)\right|=1$. 
Лемма 10. 1. Пусть $G(\varepsilon, \mu)=\{q\}$, тогда при $\varepsilon \geqslant \varepsilon_{0}$ для любого $q_{1} \in \mathbb{Z}_{+}$

$$
\left|\frac{\partial p_{q_{1}}}{\partial \mu}-\frac{\partial p_{q_{1}+1}}{\partial \mu}+1\right| \leqslant C e^{-2 \varepsilon} .
$$

2. Пусть $\{q, q+1\} \subset G(\varepsilon, \mu)$, тогда при $\varepsilon \geqslant \varepsilon_{0}$

$$
\left|\frac{\partial p}{\partial \mu}\right|_{(\varepsilon, \mu) \in \partial_{q} \mathcal{M}}-\left.\frac{\partial p}{\partial \mu}\right|_{(\varepsilon, \mu) \in \partial_{q+1} \mathcal{M}}+1 \mid \leqslant C e^{-2 \varepsilon},
$$

где $\partial p /\left.\partial \mu\right|_{(\varepsilon, \mu) \in \partial_{q} \mathcal{M}}-$ предельное значение $\partial p / \partial \mu n p u\left(\varepsilon_{1}, \mu_{1}\right) \underset{\left(\varepsilon_{1}, \mu_{1}\right) \in \partial_{q} \mathcal{M}}{\longrightarrow}(\varepsilon, \mu)$.

При $G(\varepsilon, \mu)=\{q\}$ из уравнения состояния имеем (см. подробнее раздел II.6)

$$
\frac{\partial \overrightarrow{\mathbf{p}}}{\partial \mu}=\frac{\partial \overrightarrow{\mathbf{h}}}{\partial \mu}+\frac{\partial \overrightarrow{\mathbf{s}}}{\partial \overrightarrow{\mathbf{p}}}(q, q) \frac{\partial \overrightarrow{\mathbf{p}}}{\partial \mu}+\frac{\partial \overrightarrow{\mathbf{s}}}{\partial \mu},
$$

где производная $\partial \overrightarrow{\mathbf{s}} / \partial \mu$ вычисляется при постоянном $\overrightarrow{\mathbf{p}}$. Используя (57) и неравенства

$$
\begin{gathered}
\left|\frac{\partial s_{q}}{\partial \mu}\right| \leqslant C q e^{-\varepsilon_{1}}, \\
\left|\frac{\partial s_{q}}{\partial p_{l}}\right| \leqslant e^{\left(C-\varepsilon_{1}\right)|q-l|}, \\
\left|\frac{\partial\left(s_{q}-s_{q+1}\right)}{\partial \mu}\right| \leqslant C e^{-\varepsilon_{1}},
\end{gathered}
$$

легко получить (55) и (56). Для доказательства (58) положим в (III.82) $\bar{\omega}_{q}^{(1), \mu}:=$ $C q e^{-\varepsilon_{1}}$, для доказательства (59) положим $\bar{\omega}_{q}^{(1), p_{l}}:=e^{\left(C_{1}-\varepsilon_{1}\right)|q-l|}$. Проверка (III.82) для получения (59) проводится, как и при доказательстве леммы 8, при помощи неравенства (50); неравенство (58) доказывается относительно просто. Чтобы доказать (60), представим разность $\partial\left(s_{q}-s_{q+1}\right) / \partial \mu$ в виде $\partial^{2} s_{q}^{\Theta} / \partial \mu \partial \Theta$, где $s_{q}^{\Theta}$ определено так же, как при доказательстве леммы 8. Возьмем $\bar{\omega}^{(2)}:=C e^{-\varepsilon_{1}}$ (мы используем теорему III.5 при $k=2), \bar{\omega}_{q}^{(1), \mu}:=C q e^{-\varepsilon_{1}} ; \bar{\omega}_{q}^{(1), \Theta}$ определяется как правая часть равенства (48). Проверить справедливость неравенств (III.82) в этом случае можно с помощью выкладок, аналогичных рассуждениям при доказательстве леммы 8.

Прежде чем сформулировать один из основных результатов, заметим, что фазовая диаграмма рассматриваемой модели в расширенном пространстве параметров $(\overrightarrow{\mathbf{h}}, \varepsilon, \mu)($ см. $(23))$ гомеоморфна плоской фазовой диаграмме, как это следует из теорем 1 и III.1. Однако не просто найти вид сечения этой фазовой диаграммы при $\overrightarrow{\mathbf{h}}=\overrightarrow{\mathbf{h}}(\varepsilon, \mu)($ см. $(22))$ и $(\varepsilon, \mu) \in \mathcal{M}$ или, говоря точнее, исследовать сужение функции $\overrightarrow{\mathbf{p}}=\overrightarrow{\mathbf{p}}(\overrightarrow{\mathbf{h}}(\varepsilon, \mu), \varepsilon, \mu)$ и определить, на какого типа области разбита плоскость $(\varepsilon, \mu), \varepsilon>\varepsilon_{0}$, линиями, на которых несколько компонент $-p_{q}$ минимальны. Это вызвано следующими причинами. Следует ожидать, что эти линии скапливаются в окрестности луча $\mu=0, \varepsilon>\varepsilon_{0}$, и тем самым расстояние между ними и угол между касательными векторами к ним становится меньше $e^{-\varepsilon_{1}}$. Таким образом, тип 


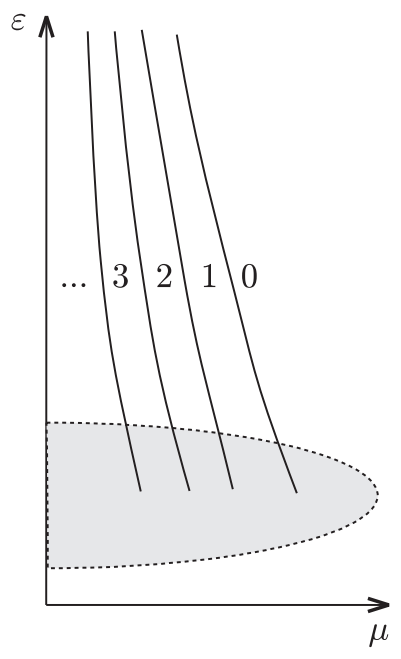

Рис. 1. Фазовая диаграмма модели Изинга в полупространстве.

фазовой диаграммы не может быть найден по низшим порядкам $e^{-\varepsilon}$. Кроме того, вектор $\overrightarrow{\mathbf{p}}$ имеет бесконечное число компонент и известен нам лишь из уравнения состояния, а само это уравнение определено при помощи функций, заданных рядами по степеням $\varphi_{\vec{\partial}}$. Конечно, для любых $\mu_{1}>0$ и $\mu>\mu_{1}$ можно взять достаточно слагаемых в уравнении состояния так, что фазовая диаграмма при $\mu>\mu_{1}$ будет определена с точностью до гомеоморфизма, но для малых $\mu_{1}$ этот путь плохо реализуем. При этом некоторые факты о фазовой диаграмме легко получить уже из доказанных результатов, в частности, из леммы 10.

Из леммы 10 вытекает следующее утверждение.

СЛЕДСТВИЕ. При любом $\mu_{1}>0$ в полуплоскости $\mu \geqslant \mu_{1}, \varepsilon>\varepsilon_{0}$ число фаз конечно. Функция $q(\varepsilon, \mu):=\max G(\varepsilon, \mu)$ есть невозрастающая функция $\mu$, которая при достаточно больших $\mu$ равна нулю. Если $|G(\varepsilon, \mu)|>1$, то в окрестности $\mu$ устойчива лишь одна фаза, т.е. при достаточно малом $\delta$ выполнено

$$
G(\varepsilon, \mu+\delta)=\max G(\varepsilon, \mu), \quad G(\varepsilon, \mu-\delta)=\min G(\varepsilon, \mu) .
$$

Для модели Изинга в полупространстве тип фазовой диаграммы при $\mu>0$ может быть определен с точностью до гомеоморфизма, если привлечь дополнительную тонкую информацию о статистических суммах, полученную при помощи корреляционных неравенств Лебовица [12], и воспользоваться критерием устойчивости фаз (теорема III.2). Следующая теорема является следствием теоремы 6 раздела 7 настоящей работы.

Теорема 2. При $\varepsilon>\varepsilon_{0}$, где $\varepsilon_{0}$ достаточно велико и зависит лишь от $\nu, в$ области $\mathcal{M}$ (41) справедливы следующие утверждения. 
1. Существует последовательность вещественно-аналитических функиий $\mu_{q}(\varepsilon), q=0,1, \ldots$, maкux, что $0<\mu_{q+1}(\varepsilon)<\mu_{q}(\varepsilon)$ (cм. puc. 1) $u$

$$
\left|\mu_{q}(\varepsilon)-e^{-2(\nu-1) \varepsilon(q+1)}\right| \leqslant e^{(C-2(\nu-1) \varepsilon(q+1))-2 \varepsilon},
$$

где $C$ зависит толъко от $\nu$.

2. На линии $\mu_{q}(\varepsilon)$ сосуществуют фазы $q$ u $q+1$, m.e. $G\left(\varepsilon, \mu_{q}(\varepsilon)\right)=\{q, q+1\}$ (см. (44)), а между линиями $\mu_{q}(\varepsilon)$ и $\mu_{q+1}(\varepsilon)$ устойчива лишь фаза q, т.е. $G(\varepsilon, \mu)=$ $\{q\}, \operatorname{eсли~}(\varepsilon, \mu) \in \partial_{q} \mathcal{M}=\left\{(\varepsilon, \mu): \mu_{q}(\varepsilon)<\mu<\mu_{q+1}(\varepsilon), \varepsilon>\varepsilon_{0}\right\}$.

3. Поверхностная свободная энергия $-p(\varepsilon, \mu)$, найденная из уравнения состояния, может быть продолжена до непрерывной функиии в $\overline{\mathcal{M}}$, совпадает с термодинамическим пределом (4) в $\mathcal{M}$ и является вещественно-аналитической функиией внутри каждого страта фазовой диаграммы (на линиях $\mu_{q}(\varepsilon)$ и между ними).

4. Производные $\partial p / \partial \varepsilon, \partial p / \partial \mu$ поверхностной свободной энергии, а также $\partial p /\left.\partial h_{q_{1}}\right|_{\overrightarrow{\mathbf{h}}=\mathbf{\mathbf { h }}(\varepsilon, \mu)}$ продолжаются до непрерывных функций в замыкании $\partial_{q} \mathcal{M}$. Эти производные продолжаются до аналитических функиий как слева, так и справа от линий $\mu=\mu_{q}(\varepsilon)$. На этих линиях разрывны $\partial p / \partial \mu, \partial p / \partial h_{q}(c \mathcal{M} .(56))$.

5. При $\mu=0$ для всех q выполнены неравенства $p_{q+1}(\varepsilon, 0)<p_{q}(\varepsilon, 0)$, и величина $-p(\varepsilon, 0)=\inf _{q}\left(-p_{q}(\varepsilon, 0)\right)$ совпадает с поверхностной свободной энергией $-p^{\mathrm{D}}(\varepsilon)$ поверхности раздела объемных (+1)- $u(-1)$-фаз модели Изинга во всем пространстве (выполнено правило Антонова, см., например, [23]). Функиия $p^{\mathrm{D}}(\varepsilon)$ вещественно аналитична при $\operatorname{Re} \varepsilon>\varepsilon_{0}$,

$$
p^{\mathrm{D}}(\varepsilon)=2 x^{2}+2 x^{3}+10 x^{4}+16 x^{5}+\frac{86}{3} x^{6}+\cdots, \quad x:=e^{-2 \varepsilon}, \quad \nu=3 .
$$

Кроме того,

$$
\left.\lim _{\substack{\mu \rightarrow+0, q(\mu) \in G(\varepsilon, \mu)}} \frac{\partial p(\overrightarrow{\mathbf{h}}, \varepsilon, \mu)}{\partial \overrightarrow{\mathbf{h}}}\right|_{\overrightarrow{\mathbf{h}}=\overrightarrow{\mathbf{h}}(\varepsilon, \mu)}=\left.\frac{\partial p^{\mathrm{D}}\left(\overrightarrow{\mathbf{h}}^{\mathrm{D}}, \varepsilon\right)}{\partial \overrightarrow{\mathbf{h}}^{\mathrm{D}}}\right|_{\overrightarrow{\mathbf{h}}^{\mathrm{D}}(\varepsilon):=h^{\mathrm{D}} \overrightarrow{\mathbf{1}}},
$$

где $-p^{\mathrm{D}}\left(\overrightarrow{\mathbf{h}}^{\mathrm{D}}, \varepsilon\right)=-\lim _{\Lambda_{S} \uparrow \mathbb{Z}_{0}^{\nu-1}}\left|\Lambda_{S}\right|^{-1} \ln \Xi^{\mathrm{D}}\left(\Lambda_{S}\right) ;$ функции $\Xi^{\mathrm{D}}\left(\Lambda_{S}\right), h^{\mathrm{D}}(\varepsilon)$ определень в (24).

Первые члены разложения в ряд величины $p^{\mathrm{D}}(\varepsilon)$ найдены в [21]. Мы получили те же члены разложения в разделе 9.

\section{5. РАСШИРЕНИЕ ОБЛАСТИ ФАЗОВЫХ ПЕРЕХОДОВ ПЕРВОГО РОДА МОДЕЛИ ИЗИНГА В $\mathbb{Z}_{+}^{\nu}$}

Чтобы расширить область фазовых переходов для поверхностных фаз модели Изинга в полупространстве, нам необходимо обобщить лемму 2 на произвольные комплексные внешние поля. 
Пусть заданы функционалы $F_{+}^{0}(\Gamma), F_{-}^{0}(\Gamma)$ на множестве контуров модели Изинга. Введем, вообще говоря, несимметричную двухфазную модель

$$
\begin{aligned}
& \Xi_{+}^{\mathrm{I}}\left(V ; \mu_{+}, \mu_{-}, F_{+}^{0}, F_{-}^{0}\right):=\sum_{X \subset V} e^{\mu_{+}\left|V \backslash V_{\mathrm{o}}(X)\right|+\mu_{-}\left|V_{\mathrm{o}}(X)\right|} F_{+}^{0 X_{\mathrm{e}}} F_{-}^{0} X \backslash X_{\mathrm{e}}, \\
& \Xi_{-}^{\mathrm{I}}\left(V ; \mu_{+}, \mu_{-}, F_{+}^{0}, F_{-}^{0}\right):=\sum_{X \subset V} e^{\mu_{+}\left|V_{\mathrm{o}}(X)\right|+\mu_{-}\left|V \backslash V_{\mathrm{o}}(X)\right|} F_{+}^{0 X \backslash X_{\mathrm{e}}} F_{-}^{0} X_{\mathrm{e}},
\end{aligned}
$$

где $X$ - набор контуров Изинга; $V_{\mathrm{o}}(X)$ - как и ранее, те узлы $V$, которые охватываются нечетным числом контуров $X ; X_{\mathrm{e}} \subset X$ - те контуры, которые охватывают четное число контуров $X$. Из определений (5) и (61) имеем

$$
\Xi_{ \pm}(V)=\Xi_{ \pm}^{\mathrm{I}}(V ; 0,-\mu, F, F), \quad F(\Gamma)=e^{-\varepsilon|\Gamma|} .
$$

Из равенства

$$
\left|V_{\mathrm{o}}(X)\right|=\sum_{\Gamma \in X_{\mathrm{e}}}|V(\Gamma)|-\sum_{\Gamma \in X \backslash X_{\mathrm{e}}}|V(\Gamma)|
$$

и определения (61) легко получить, что для любых $\alpha_{+}, \alpha_{-}$

$$
\begin{aligned}
& \Xi_{+}^{\mathrm{I}}\left(V ; \mu_{+}, \mu_{-}, F_{+}^{0}, F_{-}^{0}\right)=e^{i \alpha_{+}|V|} \Xi_{+}^{\mathrm{I}}\left(V ; \mu_{+}-i \alpha_{+}, \mu_{-}-i \alpha_{-}, F_{+, \alpha}^{0}, F_{-, \alpha}^{0}\right), \\
& \Xi_{-}^{\mathrm{I}}\left(V ; \mu_{+}, \mu_{-}, F_{+}^{0}, F_{-}^{0}\right)=e^{i \alpha_{-}|V|} \Xi_{-}^{\mathrm{I}}\left(V ; \mu_{+}-i \alpha_{+}, \mu_{-}-i \alpha_{-}, F_{+, \alpha}^{0}, F_{-, \alpha}^{0}\right),
\end{aligned}
$$

где

$$
F_{ \pm, \alpha}^{0}(\Gamma)=F_{ \pm}^{0}(\Gamma) e^{-i\left(\alpha_{ \pm}-\alpha_{\mp}\right)|V(\Gamma)|} .
$$

Формулы (64) и (65) суть частные случаи формул (III.52) и (III.51), соответственно, для двухфазных контурных моделей. Отметим, что статистические суммы в правой части (64) несимметричны относительно взаимной замены + на - даже при симметричных начальных функционалах.

Пусть $\left(p_{+}, p_{-}\right)-$комплексный вектор и $\alpha_{ \pm}=\operatorname{Im} p_{ \pm}$. Для двухфазной модели со статистическими суммами из правых частей равенств (64) проведем построения раздела I.4 для вектора свободной энергии $\left(\operatorname{Re} p_{+}, \operatorname{Re} p_{-}\right)$. Пусть устойчива фаза + , т.е. $\operatorname{Re} p_{+} \geqslant \operatorname{Re} p_{-}$. Тогда уравнение состояния принимает вид $\operatorname{Re} p_{+}=\mu_{+}-i \operatorname{Im} p_{+}+$ $s\left(\mathcal{F}_{+}\right), \operatorname{Re} p_{-}=\mu_{-}-i \operatorname{Im} p_{-}+s\left(\mathcal{F}_{-}\right)$или

$$
p_{+}=\mu_{+}+s\left(\mathcal{F}_{+}\right), \quad p_{-}=\mu_{-}+s\left(\mathcal{F}_{-}\right) .
$$

Исключая функционалы $\mathcal{F}_{+,-}(\Gamma)$ из контурных уравнений (III.17), получаем

$$
\begin{aligned}
& \mathcal{F}_{+}(\Gamma)=F_{+, \alpha}^{0}(\Gamma) \frac{\Delta_{-}(V(\Gamma))}{\Delta_{+}(V(\Gamma))} \sigma_{-}(V(\Gamma)), \\
& \mathcal{F}_{-}(\Gamma)=F_{-, \alpha}^{0}(\Gamma) \frac{\Delta_{+}(V(\Gamma))}{\Delta_{-}(V(\Gamma))} \sigma_{-}(V(\Gamma)), \\
& \sigma_{-}(V)=\sum_{X \subset V}\left(F_{-, \alpha}^{0} \frac{\Delta_{+}}{\Delta_{-}}\right)^{X} \varphi_{\partial V \cup X} \delta_{-}(\partial V \cup X),
\end{aligned}
$$


где соответствующие величины определены в (8), (9), (12). Пусть $\Delta p=p_{-}-p_{+}$, $\Delta \mu=\mu_{-}-\mu_{+}, \Delta s=s\left(\mathcal{F}_{-}\right)-s\left(\mathcal{F}_{+}\right)$. Из уравнения состояния получаем

$$
\Delta \mu=\Delta p-\Delta s(\Delta p)
$$

$\mathrm{B}$ точке сосуществования фаз, где $\operatorname{Re} p_{+}=\operatorname{Re} p_{-}$, контурные уравнения и уравнение линии сосуществования двух фаз примут вид

$$
\begin{gathered}
\mathcal{F}_{+}(\Gamma)=F_{+, \alpha}^{0}(\Gamma) \frac{\Delta_{-}(V(\Gamma))}{\Delta_{+}(V(\Gamma))}, \quad \mathcal{F}_{-}(\Gamma)=F_{-, \alpha}^{0}(\Gamma) \frac{\Delta_{+}(V(\Gamma))}{\Delta_{-}(V(\Gamma))} ; \\
\Delta \mu=i \operatorname{Im} \Delta p-\left.\Delta s(i \operatorname{Im} \Delta p)\right|_{\operatorname{Re} \Delta p=0} .
\end{gathered}
$$

Исключая параметр $\operatorname{Im} \Delta p$ из (70), линию сосуществования фаз запишем в явном виде $\operatorname{Re} \Delta \mu=\mu_{\mathrm{R}}(\operatorname{Im} \Delta \mu)$. Заметим, что при $F_{+}^{0}(\Gamma)=F_{-}^{0}(\Gamma)^{*}$ (в частности, для вещественных $\varepsilon$ в модели Изинга) выполнено $F_{+, \alpha}^{0}(\Gamma)=F_{-, \alpha}^{0}(\Gamma)^{*}$. Таким образом, $\mathcal{F}_{+}=\mathcal{F}_{-}^{*}, s\left(\mathcal{F}_{+}\right)=s\left(\mathcal{F}_{-}\right)^{*}$. Следовательно, линия сосуществования фаз есть прямая

$$
\left.\operatorname{Re} \Delta \mu(\Delta p)\right|_{\operatorname{Re} \Delta p=0}=0, \quad \text { если } \quad F_{+}^{0}(\Gamma)=F_{-}^{0}(\Gamma)^{*} .
$$

ТЕорема 3. Область $\left|F_{+}^{0}(\Gamma)\right| \leqslant e^{-\varepsilon_{0}|\Gamma|},\left|F_{-}^{0}(\Gamma)\right| \leqslant e^{-\varepsilon_{0}|\Gamma|}$ является областъю фазовых переходов первого рода для двухфазной модели (62); в этой области выполнена теорема III.4 (и теоремы I.4-I.7). В частности, имеют место следующие утверждения. Отображение (68) $\Delta p \rightarrow \Delta \mu$ переводит комплексную плоскость в комплексную плоскость, является аналитическим вне линии фазовых переходов (70) и $C^{\infty}$-гладким на самой линии; производная этого отображения отличается от 1 не более, чем на $C e^{-2 \nu \varepsilon_{0}}$, и продолюается до непрерывной функиии в замыкании каждой части плоскости; $C^{\infty}$-гладкая линия (70) делит плоскость $\Delta \mu$ на две части, в каждой из которых устойчива одна из фаз. Решение контурных уравнений (67) (на линии сосуществования фаз контурные уравнения имеют вид (69)) u функции s, задающие уравнение состояния (66), разлагаются в сходящиеся ряды по степеням $F_{ \pm}^{0}(\Gamma)$. В области устойчивости фазы $+n p u \operatorname{Re} \Delta \mu \geqslant \mu_{\mathrm{R}}(\operatorname{Im} \Delta \mu) \partial л я$ статистических сумм справедливы представления

$$
\begin{aligned}
& \Xi_{+}\left(V ; \mu_{+}, \mu_{-}, F_{+}^{0}, F_{-}^{0}\right)=e^{p_{+}|V|} \Delta_{+}(V), \\
& \Xi_{-}\left(V ; \mu_{+}, \mu_{-}, F_{+}^{0}, F_{-}^{0}\right)=e^{\left(\operatorname{Re} p_{+}+i \operatorname{Im} p_{-}\right)|V|} \Delta_{-}(V) \sigma_{-}(V) .
\end{aligned}
$$

При этом $\left|\sigma_{-}(V)\right| \leqslant 1$. Сходится кластерное разложение

$$
\begin{aligned}
\ln \frac{\mathcal{F}_{+}(\Gamma)}{\mathcal{F}_{-}(\Gamma)}-\ln \frac{F_{+, \alpha}^{0}(\Gamma)}{F_{-, \alpha}^{0}(\Gamma)} & =2 \ln \frac{\Delta_{-}(V(\Gamma))}{\Delta_{+}(V(\Gamma))}= \\
& =2 \sum X: V(X) \cap \Gamma \neq \varnothing \frac{|V(X) \cap V(\Gamma)|}{|V(\Gamma)|}\left(\varphi_{-}^{0}(X)-\varphi_{+}^{0}(X)\right) .
\end{aligned}
$$

На линии сосуществования фаз $\sigma_{-}(V)=1$; термодинамические предель поверхностной свободной энергии, найденные при помощи статистических сумм (61),

4 Теоретическая и математическая физика, т. 153, № 2, 2007 г. 
совпадают на линии сосуществования фаз лишь в точке $\Delta \mu=-\Delta s(0)$. Для модели, в которой $F_{+}^{0}(\Gamma)=F_{-}^{0}(\Gamma)^{*}$, в частности, для вещественных в в модели Изинга, линия сосуществования фаз есть прямая $\operatorname{Re} \Delta \mu=0$.

Конечно, теорема 3 является прямым следствием теоремы III.4 и соотношений (66)-(70). Отметим, что в модели Изинга при комплексном $\varepsilon$ линия сосуществования фаз не является прямой, хотя и слабо от нее отличается.

Модель (61), в которой $F_{+}^{0}(\Gamma)=0, \mu_{+}=0, \Xi_{+}(V)=1, p_{+}=0$, называют моделью внешних контуров. В работе [35] модель внешних контуров используется для построения обобщения теории Пирогова-Синая. В модели внешних контуров уравнение состояния и контурные уравнения имеют вид $p_{-}=-\mu+s_{-}\left(\mathcal{F}_{-}\right)$, $\mathcal{F}_{-}(\Gamma)=F_{-, \operatorname{Im} p_{-}(\mu)}^{0}(\Gamma) \Delta_{-}(V(\Gamma))^{-1}$, а точка сосуществования фаз для вещественных параметров определяется равенством $\mu=s_{-}\left(\mathcal{F}_{-}(0)\right)$, где, как и выше, $s_{-}\left(\mathcal{F}_{-}\right)$ разлагается в сходящийся ряд по степеням $F_{-}^{0}$.

Для расширения области фазовых переходов в модели Изинга в полупространстве воспользуемся в (7) представлением (72). Тогда вместо (14) получим

$$
\begin{aligned}
\Xi_{q}^{N}\left(\Lambda_{S}\right)= & \sum_{\substack{\Gamma_{\infty}^{q}, X_{1}: \Gamma_{\infty}^{q} \backslash \Gamma^{q, 0} \subset \Lambda, X_{1} \subset \Lambda_{\downarrow}\left(\Gamma_{\infty}^{q}\right)}} F_{-, \alpha}^{0}{ }^{X_{1}} e^{\Delta p\left|\Lambda_{\downarrow}\left(\Gamma_{\infty}^{q}\right)\right|} \widetilde{\varphi}_{X_{1}} \exp \left\{-\varepsilon\left(\left|\Gamma_{\infty}^{q} \cap \Lambda\right|-\left|\Lambda_{S}\right|\right)-\right. \\
& \left.-\sum_{X \subset \Lambda: X \cap\left(\Gamma_{\infty}^{q} \cup X_{1}\right) \neq \varnothing}^{0}\left[\frac{\left|V(X) \cap \Lambda_{\uparrow}\left(\Gamma_{\infty}^{q}\right)\right|}{|V(X)|} \varphi_{+}^{0}(X)+\frac{\left|V(X) \cap \Lambda_{\downarrow}\left(\Gamma_{\infty}^{q}\right)\right|}{|V(X)|} \varphi_{-}^{0}(X)\right]\right\},
\end{aligned}
$$

где $\widetilde{\varphi}_{X_{1}}$ зависит от $b=e^{\operatorname{Re} p_{-}-\operatorname{Re} p_{+}}$.

Представление (73) для статистических сумм отличается лишь небольшой модификацией взаимодействия контуров и заменой $q \mu \rightarrow q \operatorname{Re} \Delta p$. Таким образом, определение 1 для статистических сумм (73) не меняется. Определение 1 позволяет рассмотреть статистические суммы (14) как многофазную контурную модель. По сравнению с тем, что сделано ранее в леммах $3-7$, изменения в формулировках и доказательствах незначительны, и мы не будем останавливаться на них. Следовательно, на основании теоремы III.4 мы можем утверждать, что область фазовых переходов первого рода для модели Изинга в полупространстве, а также область кусочной аналитичности поверхностной свободной энергии расширяются до $\operatorname{Re} \mu>\mu_{\mathrm{R}}(\operatorname{Im} \mu, \varepsilon), \operatorname{Re} \varepsilon>\varepsilon_{0}$. Сформулируем результат в виде теоремы.

ТеоремА 4. Существуют константа $\varepsilon_{0}$, зависящая лишь от $\nu$, такая, что область

$$
\mathcal{M}_{\mathbb{C}}=\left\{(\varepsilon, \mu) \in \mathbb{C}^{2}: \operatorname{Re} \mu>\mu_{\mathrm{R}}(\operatorname{Im} \mu, \varepsilon), \operatorname{Re} \varepsilon>\varepsilon_{0}\right\}
$$

является областъю фазовых переходов первого рода относительно введенных фаз модели Изинга в $\mathbb{Z}_{+}^{\nu}$ с неустойчивым граничным условием. В замыкании области $\mathcal{M}_{\mathbb{C}}$ величи́ны $\bar{\omega}, \bar{\omega}_{p}^{\prime}, \bar{\omega}_{\varepsilon}^{\prime}$ (см. теоремы I.4-I.7) не превосходят $C e^{-\nu \operatorname{Re} \varepsilon}$, в $\mathcal{M}_{\mathbb{C}}$ справедливо неравенство $\bar{\omega}_{\mu}^{\prime}<C e^{-\nu \varepsilon} / \operatorname{Re}\left(\mu-\mu_{\mathrm{R}}(\operatorname{Im} \mu, \varepsilon)\right)$. Решение уравнения состояния и контурных уравнений существует и слабо непрерывно по $\overrightarrow{\mathbf{h}}, \varepsilon, \mu$ при 
$\overrightarrow{\mathbf{h}} \in \mathbb{C}^{\mathbb{Z}_{+}}\left(\operatorname{Re} h_{q}-\right.$ ограниченная сверху последовательность $) u(\varepsilon, \mu) \in \mathcal{M}_{\mathbb{C}}$. При $(\varepsilon, \mu) \in \mathcal{M}_{\mathbb{C}} u \overrightarrow{\mathbf{h}}=\overrightarrow{\mathbf{h}}(\varepsilon, \mu)$ уравнение состояния (42) определяет поверхностную свободную энергию, совпадающую с термодинамическим пределом (4) для устойчивых q. Поверхностная свободная энергия является кусочно-аналитической функилей в $\mathcal{M}_{\mathbb{C}}$, и каждый страт фазовой диаграммы является аналитическим множеством, а фазовая диаграмма определяется функиией

$$
G(\varepsilon, \mu)=\left\{q \in \mathbb{Z}_{+}: \min _{q_{1}}\left(-\operatorname{Re} p_{q_{1}}(\varepsilon, \mu)\right)=-\operatorname{Re} p_{q}(\varepsilon, \mu)\right\} .
$$

\section{6. ПРОИЗВОЛЬНОЕ ВНЕШНЕЕ ПОЛЕ В НУЛЕВОМ СЛОЕ}

Введение независимого внешнего поля в нулевой слой эквивалентно введению взаимодействия нулевого слоя спинов с подложкой. Случай произвольного взаимодействия нулевого слоя спинов с подложкой представляет самостоятельный интеpec, и рассмотрение его необходимо для доказательства теоремы 2. Заметим также, что внешнее поле $\mu_{0}$ в нулевом слое позволяет гладко перейти от неустойчивого граничного условия на подложке к устойчивому условию. Действительно, при $\mu_{0}=\mu$ граничное условие на подложке неустойчиво (спины подложки равны -1$)$, при $\mu_{0}=\mu+2 \varepsilon$ граничное условие на подложке устойчиво (можно поменять спины подложки с -1 на +1 и одновременно взять $\left.\mu_{0}=\mu\right)$.

Нам удобно иметь несколько иное, чем (14), представление "поверхностных" статистических сумм. Как и раньше, рассмотрим представление (7) для статистических сумм $\Xi_{q}\left(\Lambda ; \mu_{0}, \mu\right)$. В (7) для $\Xi_{-}\left(\Lambda_{\downarrow}\left(\Gamma_{\infty}^{q}\right)\right)$ воспользуемся представлением (6) и в каждом $X_{1}$ в (6) выделим контуры, для которых $V(\Gamma) \cap \Lambda_{S} \neq \varnothing$, их совокупность обозначим $X_{0}$. Заметим, что $X_{0} \subset \operatorname{ex} X_{1}$ (ex $X_{1}-$ внешние контуры $X_{1}$ ), так как в $\mathbb{Z}_{+}^{\nu}$ не может быть контуров, охватывающих $X_{0}$. Таким образом,

$$
\Xi_{-}\left(\Lambda_{\downarrow}\right)=\sum_{X_{0} \subset \Lambda_{\downarrow}: V(\Gamma) \cap \Lambda_{S} \neq \varnothing \forall \Gamma \in X_{0}}\left[F(\Gamma) \Xi_{+}(V(\Gamma))\right]^{X_{0}} \Xi_{-}\left(\Lambda_{\downarrow} \backslash V\left(X_{0}\right) \backslash \Lambda_{S}\right) .
$$

Подставив это разложение в (7) и используя (10), получаем

$$
\begin{aligned}
\Xi_{q}\left(\Lambda ; \mu_{0}, \mu\right)=\sum & e^{-\varepsilon\left|\Gamma_{\infty}^{q} \cap \Lambda\right|} \Xi_{+}\left(\Lambda_{\uparrow}\left(\Gamma_{\infty}^{q}\right)\right) \Xi_{+}\left(\Lambda_{\downarrow}\left(\Gamma_{\infty}^{q}\right) \backslash V\left(X_{0}\right) \backslash \Lambda_{S}\right) \times \\
& \times\left[\Xi_{+}(V(\Gamma)) F(\Gamma)\right]^{X_{0}} \sigma\left(\Lambda_{\downarrow}\left(\Gamma_{\infty}^{q}\right) \backslash V\left(X_{0}\right) \backslash \Lambda_{S}\right),
\end{aligned}
$$

где суммирование ведется при следующих условиях: $\Gamma_{\infty}^{q}, X_{0}$ таковы, что $\Gamma_{\infty}^{q} \backslash \Gamma_{\infty}^{q, 0} \subset$ $\Lambda, X_{0} \subset \Lambda_{\downarrow}\left(\Gamma_{\infty}^{q}\right), V(\Gamma) \cap \Lambda_{S} \neq \varnothing$ для всех $\Gamma \in X_{0}$.

Положим

$$
\Xi_{q}^{N}\left(\Lambda_{S} ; \mu_{0}, \mu\right):=\frac{e^{\varepsilon\left|\Lambda_{S}\right|} \Xi_{q}\left(\Lambda ; \mu_{0}, \mu\right)}{\Xi_{+}(\Lambda ; \mu, \mu)} .
$$

Из (77), (76), (8) и (12) находим

$$
\Xi_{q}^{N}\left(\Lambda_{S} ; \mu_{0}, \mu\right)=\sum_{\mathrm{A}} \exp \left\{-\varepsilon\left(\left|\Gamma_{\infty}^{q} \cap \Lambda\right|-\left|\Lambda_{S}\right|\right)-\sum_{\mathrm{B}}^{0} \varphi^{0}(X)\right\} F^{X_{0} \cup X_{1}} \widetilde{\varphi}_{X_{1}} b^{\Lambda_{\downarrow} \backslash V\left(X_{0}\right)}
$$


где суммирование ведется при следующих условиях:

условие A: $\Gamma_{\infty}^{q} \backslash \Gamma_{\infty}^{q, 0} \subset \Lambda, X_{0} \subset \Lambda_{\downarrow}\left(\Gamma_{\infty}^{q}\right), X_{1} \subset \Lambda_{\downarrow}\left(\Gamma_{\infty}^{q}\right) \backslash V\left(X_{0}\right) \backslash \Lambda_{S}, V(\Gamma) \cap \Lambda_{S} \neq \varnothing$ для любых $\Gamma \in X_{0}$;

условие В: $X \subset \Lambda: X \cap\left(X_{0} \cup X_{1} \cup \Gamma_{\infty}^{q} \cup \Gamma_{\infty}^{0,0}\right) \neq \varnothing$.

$\mathrm{B}$ представлении (78) лишь $b^{\Lambda_{\downarrow} \backslash V\left(X_{0}\right)}$ зависит от $\mu_{0}, b^{V}:=e^{-\mu_{0}\left|V \cap \Lambda_{S}\right|-\mu\left|V \backslash \Lambda_{S}\right|}$. Для статистических сумм (78) необходимо модифицировать определение 1.

ОПРЕДЕЛЕНИЕ 2. Для модели со статистическими суммами (78) множкество фаз есть $\mathbb{Z}^{+}$, множество границ с граничным условием $q$ совпадает с множеством троек $\left(\Gamma_{\infty}^{q}, X_{0}, X_{1}\right)$ таких, что при некотором $\Lambda$ выполнено условие "А" суммирования в (78). Носитель границы $\vec{\partial} \in \gamma_{q}$ есть $V_{\vec{\partial}}:=V_{\vec{\partial}_{1}} \cup \operatorname{pr} X_{0}$, где $V_{\vec{\partial}_{1}}$ - носитель границы $\vec{\partial}_{1}:=\left(\Gamma_{\infty}^{q}, X_{1}\right)$ определения 1 .

Величины $h_{q}^{\Lambda}, H_{0}^{\Lambda}(\overrightarrow{\boldsymbol{\partial}})$ для статистических сумм $\Xi_{q}^{N}\left(\Lambda_{S} ; \mu_{0}, \mu\right)$ имеют вид

$$
\begin{gathered}
h_{0}^{\Lambda}(x):=h_{0}^{1, \Lambda}(x):=-\sum_{X \subset \Lambda: \min _{1} X \cap \Gamma_{\infty}^{0,0}=x} \varphi^{0}(X), \\
h_{q}^{\Lambda}(x):=-\mu_{0}-\mu(q-1)-\sum_{X \subset \Lambda: \min _{1} \operatorname{pr}\left(X \cap\left(\Gamma_{\infty}^{q, 0} \cup \Gamma_{\infty}^{0,0}\right)\right)=x} \varphi^{0}(X), \quad q \geqslant 1, \\
e^{-H_{0}^{\Lambda}(\overrightarrow{\boldsymbol{\partial}})}:=e^{-\left(\varepsilon\left|\Gamma_{\infty}^{q} \cap \Lambda\right|-\left|\Lambda_{S}\right|\right)} F^{X_{0} \cup X_{1}} b^{\left(\Lambda_{\downarrow}(\overrightarrow{\boldsymbol{\partial}}) \backslash V\left(X_{0}\right)\right) \cap \operatorname{pr}^{-1}} V_{\overrightarrow{\boldsymbol{\partial}}} \widetilde{\varphi}_{X_{1}} ;
\end{gathered}
$$

величины $W^{\Lambda}(V \mid \overrightarrow{\boldsymbol{\partial}})$ определены теми же формулами (21) за исключением того, что теперь $k_{i}(\overrightarrow{\boldsymbol{\partial}}) \mu$ надо заменить на $\mu_{0}+\left(k_{i}(\overrightarrow{\boldsymbol{\partial}})-1\right) \mu$, если $k_{i}(\overrightarrow{\boldsymbol{\partial}})>0$ и на нуль, если $k_{i}(\vec{\partial})=0$

$$
\Theta_{\Lambda}(X \mid \overrightarrow{\boldsymbol{\partial}}):= \begin{cases}1, & X \cap\left(X_{0} \cup X_{1} \cup \Gamma_{\infty}^{q} \cup \Gamma_{\infty}^{0,0}\right) \neq \varnothing, \quad X \subset \Lambda, \\ 0, & X \cap\left(X_{0} \cup X_{1} \cup \Gamma_{\infty}^{q} \cup \Gamma_{\infty}^{0,0}\right)=\varnothing .\end{cases}
$$

Сравнивая определения (79), (24) и (22), легко видеть, что

$$
h_{\infty}^{1}:=\lim _{q \rightarrow \infty} h_{q}^{1}=h_{0}^{1}+h^{\mathrm{D}} .
$$

Подчеркнем, что от $\mu_{0}$ зависит лишь $h_{q}^{\Lambda}, H_{0}^{\Lambda}(\overrightarrow{\boldsymbol{\partial}})$. Поэтому доказательства лемм 4-6 практически не меняются, неравенство (39) леммы 7 также справедливо в области $\varepsilon>\varepsilon_{0}, \mu_{0} \in \mathbb{R}, \mu \geqslant 0$, так как основная часть доказательства та же, что и в лемме 7 . По существу, отличие заключается лишь в том, что $\sup h_{q}$ в неравенстве (39) надо заменить на $\max _{q>1}\left\{0,-\mu_{0}-(q-1) \mu\right\}$; данный максимум при отрицательных $\mu_{0}$ равен $\mu_{0}$, при положительных $\mu$ равен нулю. Из этого замечания и определения $H_{0}^{\Lambda}(\overrightarrow{\boldsymbol{\partial}})$ видно, что правая часть (36) содержит зависимость от $\mu_{0}$ в виде множителя $e^{-\left|\mu_{0}\right| a}, a \geqslant 0$. Таким образом, справедливо обобщение теоремы 1.

Tеорема 5. Существует $\varepsilon_{0}$ такое, что область

$$
\mathcal{M}:=\left\{\left(\varepsilon, \mu_{0}, \mu\right): \varepsilon>\varepsilon_{0}, \mu>0\right\}
$$

является областью фазовых переходов первого рода для статистических сумм (78) с множеством фаз, введенных в определении 2. В замыкании $\mathcal{M}$ величи́ны $\bar{\omega}, \bar{\omega}_{p}^{\prime}$, $\bar{\omega}_{\varepsilon}^{\prime}, \bar{\omega}_{\mu_{0}}^{\prime}$ не превосходят $C e^{-2(\nu-1) \varepsilon}$, кроме того, $\bar{\omega}_{\mu}^{\prime}<\infty$ в $\mathcal{M}$. 
Неравенства леммы 10 также можно обобщить.

ЛЕмма 11. Справедливы следующие неравенства.

1. Пусть $G\left(\varepsilon, \mu_{0}, \mu\right)=\{q\}$, тогда при $\varepsilon \geqslant \varepsilon_{0}\left(\varepsilon_{1}:=2(\nu-1) \varepsilon\right)$

$$
\left|\frac{\partial p_{q_{1}}}{\partial \mu}-\frac{\partial p_{q_{1}+1}}{\partial \mu}+1\right| \leqslant C e^{-\varepsilon_{1}}, \quad q_{1}>0 .
$$

2. Пусть $\{q, q+1\} \subset G\left(\varepsilon, \mu_{0}, \mu\right)$, тогда при $\varepsilon \geqslant \varepsilon_{0}$

$$
\begin{aligned}
& \left|\frac{\partial p}{\partial \mu}\right|_{\left(\varepsilon, \mu_{0}, \mu\right) \in \partial_{q} \mathcal{M}}-\left.\frac{\partial p}{\partial \mu}\right|_{\left(\varepsilon, \mu_{0}, \mu\right) \in \partial_{q+1} \mathcal{M}}+1 \mid \leqslant C e^{-\varepsilon_{1}}, \quad q>0, \\
& \left|\frac{\partial p}{\partial \mu_{0}}\right|_{\left(\varepsilon, \mu_{0}, \mu\right) \in \partial_{q} \mathcal{M}}-\left.\frac{\partial p}{\partial \mu_{0}}\right|_{\left(\varepsilon, \mu_{0}, \mu\right) \in \partial_{q+1} \mathcal{M}}+1 \mid \leqslant C e^{-\varepsilon_{1},} \quad q=0 .
\end{aligned}
$$

Кроме того, в $\mathcal{M}$ выполняется неравенство

$$
\left|\frac{\partial s_{q}}{\partial \mu}\right| \leqslant C q e^{-\varepsilon_{1}}, \quad q>0 .
$$

\section{7. НЕРАВЕНСТВА ЛЕБОВИЦА И АНАЛИЗ ФАЗОВОЙ ДИАГРАММЫ}

Пусть $\bar{\Lambda}=\Lambda \cup \partial \bar{\Lambda} \subset \mathbb{Z}^{\nu}$, где $\partial \bar{\Lambda}$ - граничные узлы $\bar{\Lambda}, \Lambda=\bar{\Lambda} \backslash \partial \bar{\Lambda}, \bar{\Lambda}-$ цилиндр с основанием $\bar{\Lambda}_{S} \subset \mathbb{Z}_{0}^{\nu-1}$ высоты $N+2$. Рассмотрим два независимых экземпляра модели Изинга в $\bar{\Lambda}$ со статистическими суммами

$$
\begin{aligned}
& \Xi(\bar{\Lambda} ; H-h):=\sum_{\sigma} \exp \left\{-\varepsilon \sum_{[i, j] \subset \bar{\Lambda}} \sigma_{i} \sigma_{j}+\sum_{i \in \bar{\Lambda}}\left(H_{i}-h_{i}\right) \sigma_{i}\right\}, \\
& \widetilde{\Xi}(\bar{\Lambda} ; H+h):=\sum_{\tilde{\sigma}} \exp \left\{-\varepsilon \sum_{[i, j] \subset \bar{\Lambda}} \tilde{\sigma}_{i} \tilde{\sigma}_{j}+\sum_{i \in \bar{\Lambda}}\left(H_{i}+h_{i}\right) \tilde{\sigma}_{i}\right\},
\end{aligned}
$$

где $[i, j]$ - пара ближайших соседей. Перейдем в этих формулах к переменным Перкуса $s_{i}:=\left(\sigma_{i}+\tilde{\sigma}_{i}\right) / 2, t_{i}:=\left(\sigma_{i}-\tilde{\sigma}_{i}\right) / 2, s_{i} t_{i}=0$. Тогда

$$
\Xi(\bar{\Lambda} ; H-h) \widetilde{\Xi}(\bar{\Lambda} ; H+h)=\sum_{s, t} \exp \left\{-\varepsilon \sum_{[i, j]}\left(s_{i} s_{j}+t_{i} t_{j}\right)+\sum_{i}\left(h_{i} t_{i}+H_{i} s_{i}\right)\right\} .
$$

Воспользуемся неравенством Лебовица [12]

$$
\left\langle t_{i}\right\rangle_{\bar{\Lambda}} \geqslant 0
$$

где $h_{j} \geqslant 0, H_{j}$ произвольно. Пусть $\tilde{h} \geqslant 0$. Выберем поля $H_{i}, h_{i}$ следующим образом: $\left.H_{i}\right|_{\partial \bar{\Lambda} \cap \mathbb{Z}_{q}^{\nu-1}}=0,\left.H_{i}\right|_{\partial \bar{\Lambda} \cap\left(\bigcup_{k>q} \mathbb{Z}_{k}^{\nu-1}\right)}=\tilde{h},\left.H_{i}\right|_{\partial \bar{\Lambda} \cap\left(\bigcup_{k<q} \mathbb{Z}_{k}^{\nu-1}\right)}=-\tilde{h},\left.h_{i}\right|_{\partial \bar{\Lambda} \backslash \mathbb{Z}_{q}^{\nu-1}}=0$, $\left.h_{i}\right|_{\partial \bar{\Lambda} \cap \mathbb{Z}_{q}^{\nu-1}}=\tilde{h},\left.H_{i}\right|_{\Lambda}=\mu_{i},\left.h_{i}\right|_{\Lambda}=0$. При таком выборе усреднение по ансамблю со 
статистической суммой $\Xi(\bar{\Lambda} ; H-h)$ при $\tilde{h}=\infty$ есть усреднение по ансамблю со статистической суммой $\Xi\left(\Lambda \mid \sigma^{q+1}\right)$, усреднение по ансамблю со статистической суммой $\widetilde{\Xi}(\bar{\Lambda} ; H+h)$ есть соответственно $\Xi\left(\Lambda \mid \sigma^{q}\right)$. Следовательно, из (85) имеем

$$
\left\langle\sigma_{i}\right\rangle_{q, \Lambda}-\left\langle\sigma_{i}\right\rangle_{q+1, \Lambda} \geqslant 0
$$

или $\partial \ln \left[\Xi_{q}^{N}\left(\Lambda_{S}\right) / \Xi_{q+1}^{N}\left(\Lambda_{S}\right)\right] / \partial \mu_{i} \geqslant 0$. Таким образом, $\Xi_{q}^{N}\left(\Lambda_{S}\right) / \Xi_{q+1}^{N}\left(\Lambda_{S}\right)$ - возрастающая функция $\mu_{i}$, где $\mu_{i}$ произвольно.

Внешние поля принимают два различных значения в 0 -слое $\Lambda$, в 1 -слое $\Lambda$ и третье значение, отличное от первых двух, во всех остальных слоях. Аргумент $\Lambda_{S}$ в статистических суммах $\Xi_{k}^{N}\left(\Lambda_{S}\right)$ будем опускать, а поля указывать, т.е. $\Xi_{k}^{N}\left(\Lambda_{S}\right)=$ : $\Xi_{k}^{N}\left(\mu_{0}, \mu_{1}, \mu\right)$. Тогда

$$
\frac{\Xi_{q}^{N}\left(-\infty, \mu_{0}, \mu\right)}{\Xi_{q+1}^{N}\left(-\infty, \mu_{0}, \mu\right)} \leqslant \frac{\Xi_{q}^{N}\left(\mu_{0}, \mu_{0}, \mu\right)}{\Xi_{q+1}^{N}\left(\mu_{0}, \mu_{0}, \mu\right)} \leqslant \frac{\Xi_{q}^{N}\left(\mu_{0}, \mu, \mu\right)}{\Xi_{q+1}^{N}\left(\mu_{0}, \mu, \mu\right)}
$$

при $\mu_{0} \leqslant \mu$. Так как $\Xi_{q}^{N}\left(-\infty, \mu_{0}, \mu\right)=\Xi_{q-1}^{N-1}\left(\mu_{0}, \mu, \mu\right)$, имеем $\Xi_{q-1}\left(\Lambda_{S}\right) \Xi_{q+1}\left(\Lambda_{S}\right) \leqslant$ $\left[\Xi_{q}\left(\Lambda_{S}\right)\right]^{2}, \mu_{0} \leqslant \mu$, где $\Xi_{q}\left(\Lambda_{S}\right):=\lim _{N \rightarrow \infty} \Xi_{q}^{N}\left(\Lambda_{S}\right)$. Аналогично получаем неравенство

$$
\Xi_{q_{1}}\left(\Lambda_{S}\right) \Xi_{q_{2}}\left(\Lambda_{S}\right) \leqslant \Xi_{l_{1}}\left(\Lambda_{S}\right) \Xi_{l_{2}}\left(\Lambda_{S}\right), \quad q_{1}<l_{1}<l_{2}<q_{2}, \quad \mu_{0} \leqslant \mu .
$$

Докажем также неравенство

$$
\left.\Xi_{q}\left(\Lambda_{S}\right)\right|_{\mu=0} \leqslant\left.\Xi_{q+1}\left(\Lambda_{S}\right)\right|_{\mu=0}, \quad \mu_{0} \leqslant 0 .
$$

Действительно, из предыдущих рассуждений следует $\Xi_{q}\left(\mu_{0}, 0,0\right) / \Xi_{q+1}\left(\mu_{0}, 0,0\right) \leqslant$ $\Xi_{q}(0,0,0) / \Xi_{q+1}(0,0,0)$. Таким образом, достаточно доказать, что правая часть последнего неравенства не превосходит единицы. Воспользуемся опять (86), положив $\mu=\infty$ во всех слоях, кроме слоев $0,1, \ldots, 2 q$, в которых оставим $\mu=0$. Тогда, использовав симметрию при замене + на - , получим единицу в правой части последнего неравенства. Итак, доказано следующее утверждение.

Лемма 12. Для статистических сумм $\Xi_{q}\left(\Lambda_{S}\right):=\lim _{N \rightarrow \infty} \Xi_{q}^{N}\left(\Lambda_{S}\right)$ выполнены неравенства (87), (88).

Проведем анализ фазовой диаграммы. Из критерия устойчивости фаз (теорема III.2) и неравенств (87), (88) вытекает следующее. Если устойчивы фазы $q_{1}, q_{2}$, то устойчивы все фазы $l$, для которых $q_{1}<l<q_{2}$ и, следовательно, $G\left(\varepsilon, \mu_{0}, \mu\right)=$ $\left\{q \in \mathbb{Z}_{+}: q_{1} \leqslant q \leqslant q_{2}\right\}, \mu_{0} \leqslant \mu$. Если при $\mu=0, \mu_{0} \leqslant 0$ устойчива фаза $q$, то устойчивы все фазы $q_{1}>q$. Из неравенств (82), (83) дополнительно имеем, что для $G\left(\varepsilon, \mu_{0}, \mu\right)=\left\{q \in \mathbb{Z}_{+}: q_{1} \leqslant q \leqslant q_{2}\right\}$ найдется малое $\delta$ такое, что $G\left(\varepsilon, \mu_{0}, \mu+\right.$ $\delta)=\left\{q_{1}\right\}, G\left(\varepsilon, \mu_{0}, \mu-\delta\right)=\left\{q_{2}\right\}$. Из этих свойств фазовой диаграммы и теоремы III.1 получаем, что существует последовательность кусочно-аналитических функций $\mu_{q}\left(\varepsilon, \mu_{0}\right), \mu_{q}(\varepsilon), q=0,1, \ldots$, таких, что

$$
\begin{gathered}
0 \leqslant \mu_{q}\left(\varepsilon, \mu_{0}\right) \leqslant \mu_{q+1}\left(\varepsilon, \mu_{0}\right), \quad 0 \leqslant \mu_{q}(\varepsilon) \leqslant \mu_{q+1}(\varepsilon), \quad \mu_{q}(\varepsilon)=\mu_{q}\left(\varepsilon, \mu_{q}(\varepsilon)\right), \\
G\left(\varepsilon, \mu_{0}, \mu_{q}\left(\varepsilon, \mu_{0}\right)\right)=\bigcup_{q_{1}: \mu_{q_{1}}\left(\varepsilon, \mu_{0}\right)=\mu_{q}\left(\varepsilon, \mu_{0}\right)}\left\{q_{1}, q_{1}+1\right\} .
\end{gathered}
$$


Так как производная от поверхностного натяжения по касательному направлению страта непрерывна (см. теорему І.3), получаем уравнение Клайперона-Клаузиса

$$
\frac{\partial \mu_{q}}{\partial \mu_{0}}=-\frac{\left\langle\sigma_{0}\right\rangle_{q}-\left\langle\sigma_{0}\right\rangle_{q+1}}{\left\langle\sigma^{\prime}\right\rangle_{q}-\left\langle\sigma^{\prime}\right\rangle_{q+1}}
$$

где $\left\langle\sigma_{0}\right\rangle_{q},\left\langle\sigma^{\prime}\right\rangle_{q}$ определены ниже в лемме 13. Из неравенства (86) получаем, что $\partial \mu_{q}\left(\varepsilon, \mu_{0}\right) / \partial \mu_{0} \leqslant 0$.

Докажем теперь, что в случае достаточно низких температур неравенство (86) является строгим при $\mu_{0} \leqslant \mu$ на линии сосуществования двух фаз, следовательно, строгим является и последнее неравенство.

Лемма 13. Пусть $\{q, q+1\} \subset G(\varepsilon, \mu)$, тогда при $\varepsilon \geqslant \varepsilon_{0}$

$$
\left\langle\sigma_{0}\right\rangle_{q}-\left\langle\sigma_{0}\right\rangle_{q+1}>0, \quad\left\langle\sigma^{\prime}\right\rangle_{q}-\left\langle\sigma^{\prime}\right\rangle_{q+1}>0
$$

где

$$
\begin{aligned}
\left\langle\sigma_{0}\right\rangle_{q} & :=\left.2 \frac{\partial p}{\partial \mu_{0}}\right|_{\left(\varepsilon, \mu_{0}, \mu\right) \in \partial_{q} \mathcal{M}}:=\lim _{\substack{N \rightarrow \infty \\
\Lambda_{S} \uparrow \mathbb{Z}_{0}^{\nu-1}}} \frac{1}{\left|\Lambda_{S}\right|} \sum_{i \in \Lambda_{S}}\left(\left\langle\sigma_{i}\right\rangle_{q, \Lambda}-\left\langle\sigma_{i}\right\rangle_{+, \Lambda}\right), \\
\left\langle\sigma^{\prime}\right\rangle_{q} & :=\left.2 \frac{\partial p}{\partial \mu}\right|_{\left(\varepsilon, \mu_{0}, \mu\right) \in \partial_{q} \mathcal{M}}=\lim _{\substack{N \rightarrow \infty, \Lambda_{S} \uparrow \mathbb{Z}_{0}^{\nu-1}}} \frac{1}{\left|\Lambda_{S}\right|} \sum_{i \in \Lambda \backslash \Lambda_{S}}\left(\left\langle\sigma_{i}\right\rangle_{q, \Lambda}-\left\langle\sigma_{i}\right\rangle_{+, \Lambda}\right),
\end{aligned}
$$

$\left\langle\sigma_{i}\right\rangle_{q, \Lambda}$ - усреднение по ансамблю со статистической суммой $\Xi\left(\Lambda \mid \sigma^{q} ; \mu_{0}, \mu\right)$.

Запишем среднее $\left\langle t_{i}\right\rangle_{\bar{\Lambda}}$ (см. (85)) в виде суммы по $V_{1}$, где $V_{1}$ - те узлы $\bar{\Lambda}$, в которых $t_{i} \neq 0$ :

$$
\left\langle t_{i_{0}}\right\rangle_{\bar{\Lambda}}=\sum_{V_{1} \subset \bar{\Lambda}}\left\langle t_{i_{0}}\right\rangle_{V_{1}, h} P\left(V_{1}\right)=\sum_{V_{1} \subset \bar{\Lambda}} \frac{\Xi^{t}\left(t_{i_{0}} ; V_{1}, h\right) \Xi^{s}\left(\bar{\Lambda} \backslash V_{1}, h\right)}{\Xi(\bar{\Lambda} ; H-h) \Xi(\bar{\Lambda} ; H+h)},
$$

где

$$
\Xi^{t}\left(t_{i_{0}} ; V, h\right)=\sum_{t: t_{j}=+1,-1} t_{i_{0}} \exp \left\{-\varepsilon \sum_{[i, j] \subset V} t_{i} t_{j}+\sum_{i \subset V} h_{i} t_{i}\right\} .
$$

Так как в силу неравенства Гриффитса [12] каждое слагаемое в (89) положительно, ограничимся в (85) суммой по объемам $V_{1}$, для которых $V_{0}:=\left\{i_{0}, i_{1}, \ldots, i_{q-1}\right\} \subset V_{1}$, $\operatorname{pr} V_{0}=i_{0} \in \mathbb{Z}_{0}^{\nu-1}, \bar{\Lambda} \backslash V_{1}$ содержит все ближайшие соседи $V_{0}$ в слоях $\mathbb{Z}_{0}^{\nu-1}, \ldots, \mathbb{Z}_{q-1}^{\nu-1}$ (множество этих узлов обозначим $V_{01}$ ). Отметим, что если $i_{0}$ и узлы $q$-го слоя несвязны в $V_{1}$, то $\left\langle t_{i}\right\rangle_{V_{1}, h}=0$. В статистической сумме $\Xi^{s}\left(\bar{\Lambda} \backslash V_{1}, h\right)$ ограничимся суммированием лишь по конфигурациям $s$, для которых $s\left(V_{01}\right)=-1$. Напомним, что пара конфигураций $\sigma=\sigma^{q+1}, \tilde{\sigma}=\tilde{\sigma}^{q}$ соответствует следующей конфигурации $s, t: s=1, t=0$ выше $(q+1)$-го слоя, $s=0, t=-1$ в $q$-м слое, $s=-1, t=0$ в нижних слоях. Сумму $\Xi^{t}\left(t_{i_{0}} ; V_{1}, h\right)$ можно представить в виде

$$
\Xi^{t}\left(t_{i_{0}} ; V_{1}, h\right)=\Xi\left(V_{1} \backslash V_{0}, h^{+, q}\right) \Xi\left(t_{i_{0}} ; V_{0} \backslash i_{q}, h^{+, q}\right)+\Xi\left(V_{1} \backslash V_{0}, h^{-, q}\right) \Xi\left(t_{i_{0}} ; V_{0} \backslash i_{q}, h^{-, q}\right),
$$

где внешнее поле $h^{+, q}$ совпадает с $h_{i}$ вне узла $i_{q}$ (см. определение $h_{i}$ при доказательстве (87)) и $h_{i_{q}}^{+, q}=-\varepsilon$, внешнее поле $h^{-, q}$ совпадает с $h_{i}$ вне узла $i_{q}$ и $h_{i_{q}}^{-, q}=\varepsilon$. 
Очевидно, что $\Xi\left(t_{i_{0}} ; V_{0} \backslash i_{q}, h^{+, q}\right)=-\Xi\left(t_{i_{0}} ; V_{0} \backslash i_{q}, h^{-, q}\right)=: d_{q}>0$, где $d_{q}$ зависит лишь от $q, \varepsilon$. Положив

$$
\begin{gathered}
\bar{V}_{0}:=V_{0} \cup V_{01} \cup i_{q}, \quad \bar{V}_{1}:=V_{1} \backslash V_{0} \backslash i_{q}, \\
\Lambda_{V_{1}}:=\Lambda \backslash V_{1} \backslash V_{01} \backslash i_{q}, \quad \Lambda_{V_{0}}:=\Lambda \backslash\left(V_{0} \cup V_{01} \cup i_{q}\right), \\
g_{q}=\frac{d_{q}}{\Xi\left(\Lambda \mid \sigma^{q}\right) \Xi\left(\Lambda \mid \sigma^{q+1}\right)},
\end{gathered}
$$

имеем

$$
\begin{aligned}
& \left\langle\sigma_{i_{0}}\right\rangle_{q, \Lambda}-\left\langle\sigma_{i_{0}}\right\rangle_{q+1, \Lambda} \geqslant g_{q} \sum\left\{\Xi^{t}\left(\bar{V}_{1} ; h^{+, q}\right) \Xi^{s}\left(\Lambda_{V_{1}} ; s\left(i_{q}\right)=0, H\right)-\right. \\
& \left.\quad-\Xi^{t}\left(\bar{V}_{1} ; h^{-, q}\right) \Xi^{s}\left(\Lambda_{V_{1}} ; s\left(i_{q}\right)=0, H\right)\right\}= \\
& =g_{q}\left\{\Xi\left(\Lambda_{V_{0}} ; \sigma^{q}\right) \Xi\left(\Lambda_{V_{0}} ; \sigma^{q+1}\right)-\Xi\left(\Lambda_{V_{0}} ; \sigma^{q}\left(\mathbb{Z}^{\nu} \backslash i_{q}\right), \sigma\left(i_{q}\right)=-1\right) \Xi\left(\Lambda_{V_{0}} ; \sigma^{q+1}\right)\right\},
\end{aligned}
$$

где суммирование идет по $V_{1}$ таким, что $V_{0} \subset V_{1}, V_{01} \subset \Lambda \backslash V_{1}, s\left(V_{01}\right)=-1$. Последнее равенство получено при помощи перехода от переменных $s, t$ к переменным $\sigma, \tilde{\sigma}$. Для доказательства леммы осталось прежде всего проверить, что

$$
\begin{gathered}
\frac{\Xi\left(\Lambda \backslash \bar{V}_{0} \mid \sigma^{q}\left(\mathbb{Z}^{\nu} \backslash i_{q}\right), \sigma\left(i_{q}\right)=-1\right)}{\Xi\left(\Lambda \backslash \bar{V}_{0} \mid \sigma^{q}\right)} \leqslant C e^{-2(\nu-1) \varepsilon}, \\
\frac{\Xi\left(\Lambda \backslash \bar{V}_{0} \mid \sigma^{q+1}\left(\mathbb{Z}^{\nu} \backslash i_{q}\right), \sigma\left(i_{q}\right)=+1\right)}{\Xi\left(\Lambda \backslash \bar{V}_{0} \mid \sigma^{q+1}\right)} \leqslant C e^{-2(\nu-1) \varepsilon} .
\end{gathered}
$$

Для статистических сумм $\Xi\left(\Lambda \backslash \bar{V}_{0} \mid \sigma^{q}\left(\mathbb{Z}^{\nu} \backslash i_{q}\right), \sigma\left(i_{q}\right)=-1\right)$ и $\Xi\left(\Lambda \backslash \bar{V}_{0} \mid \sigma^{q+1}\left(\mathbb{Z}^{\nu} \backslash i_{q}\right)\right.$, $\left.\sigma\left(i_{q}\right)=+1\right)$ введем представления вида (76) и затем (78). Легко видеть, что в представлении для $\Xi\left(\Lambda \backslash \bar{V}_{0} \mid \sigma^{q}\left(\mathbb{Z}^{\nu} \backslash i_{q}\right), \sigma\left(i_{q}\right)=-1\right)$ каждая граница $\vec{\partial}$ охватывает $i_{0}$, откуда мы получаем нужное неравенство аналогично тому, как это делалось в лемме 8. Положительность отношений $\Xi\left(\Lambda \backslash \bar{V}_{0} \mid \sigma^{q}\right) / \Xi\left(\Lambda \mid \sigma^{q}\right)$ и $\Xi\left(\Lambda \backslash \bar{V}_{0} \mid \sigma^{q+1}\right) / \Xi\left(\Lambda \mid \sigma^{q+1}\right)$ легко вытекает из устойчивости фаз $q$ и $q+1$ и представления (III.23). Второе неравенство леммы доказывается аналогично. Таким образом, $\mu_{q}\left(\varepsilon, \mu_{0}\right)$ есть убывающая функция $\mu_{0}$.

Из явного вида $h_{q}$, см. (79), и оценки $\left|s_{q}\right| \leqslant C e^{-\varepsilon_{1}}$, следует, что при $\mu>C e^{-\varepsilon_{1}}$ может быть устойчива фаза 0 или фаза 1 , отсюда же получаем, что при $\mu_{0}>C e^{-\varepsilon_{1}}$ и любых $\mu$ может быть устойчива лишь фаза 0 , а при $\mu_{0}<-C_{2} e^{-\varepsilon_{1}}$ и $\mu>C_{3} e^{-\varepsilon_{1}}-$ лишь фаза 1. В то же время из теоремы 4 следует, что внутри 0-страта $\left\langle\sigma^{\prime}\right\rangle_{0} \underset{\mu \rightarrow \infty}{\rightarrow} 0$, внутри 1-страта $\left\langle\sigma^{\prime}\right\rangle_{1} \underset{\mu \rightarrow \infty}{\rightarrow} 0$. Заметим также, что неравенство (83) можно записать в виде $\left|\left\langle\sigma_{0}\right\rangle_{0}-\left\langle\sigma_{0}\right\rangle_{1}-2\right|<C e^{-\varepsilon_{1}}$.

Из предыдущих соображений и формул $(77),(78)$ имеем $\left.\mu_{q}\left(\varepsilon, \mu_{0}\right)\right|_{q=0} \underset{\mu_{0} \rightarrow 0}{\rightarrow} \infty$, при этом $\left.\mu_{q}\left(\varepsilon, \mu_{0}\right)\right|_{q=0}$ определено при $0<\mu_{0}<\left.\mu_{q}(\varepsilon)\right|_{q=0},\left.\mu_{q}\left(\varepsilon, \mu_{0}\right)\right|_{q=0}>\mu_{q_{1}}(\varepsilon), q_{1}>0$.

Из (76), (77), (80) легко получить равенство

$$
\left.e^{2 \mu_{0}\left|\Lambda_{S}\right|} \Xi_{q}\left(\Lambda ; \mu_{0}, \mu\right)\right|_{\mu_{0}=-\infty}=\Xi_{q-1}\left(T^{-1}\left(\Lambda \backslash \Lambda_{S}\right) ; \mu, \mu\right) .
$$


Вследствие слабой непрерывности решений контурных уравнений и уравнения состояния из равенства (90) получаем $\left.\mu_{q}\left(\varepsilon, \mu_{0}\right)\right|_{\mu_{0}=-\infty}=\mu_{q-1}(\varepsilon)$. Из последнего равенства, формулы (90) и тождества $\mu_{q}(\varepsilon)=\mu_{q}\left(\varepsilon, \mu_{q}(\varepsilon)\right)$ находим, что $\mu_{q}(\varepsilon)<\mu_{q-1}(\varepsilon)$ и, следовательно, $\mu_{q}\left(\varepsilon, \mu_{0}\right)<\mu_{q-1}\left(\varepsilon, \mu_{0}\right)$, если $\mu_{0} \leqslant \mu$. Так как $p_{q}\left(\varepsilon, \mu, \mu_{q}\left(\varepsilon, \mu_{0}\right)\right)=$ $p_{q+1}\left(\varepsilon, \mu, \mu_{q}\left(\varepsilon, \mu_{0}\right)\right)$, из (88) и (82) получаем $p_{q+1}\left(\varepsilon, \mu_{0}, 0\right)<p_{q}\left(\varepsilon, \mu_{0}, 0\right), \mu_{0} \leqslant 0$. Так как при $\mu=0$ для величин $p_{q}, h_{q}$ существует предел при $q \rightarrow \infty$, то существует и предел $s_{\infty}:=\lim _{q \rightarrow \infty} s_{q}(\varepsilon, 0,0)$.

Обозначим верхнюю грань тех $\mu_{0}$, для которых $\sup _{q}\left(-p_{q}\left(\varepsilon, \mu_{0}, 0\right)\right)>-p_{q}\left(\varepsilon, \mu_{0}, 0\right)$ для всех $q$, как $\mu_{0, \infty}^{* *}(\varepsilon)$. Мы предполагаем, что это же неравенство верно для всех $\mu_{0}<\mu_{0, \infty}^{* *}(\varepsilon)$ и, более того, $\mu_{0, \infty}^{* *}(\varepsilon)$ является решением уравнения $\mu_{0, \infty}^{* *}(\varepsilon)=$ $s_{\infty}(\varepsilon, 0,0)-s_{0}\left(\varepsilon, \mu_{0, \infty}^{* *}(\varepsilon), 0\right)$, где $s_{\infty}(\varepsilon, 0,0)=\lim _{q \rightarrow \infty}\left(p_{q}(\varepsilon, 0,0)-h_{q}(\varepsilon, 0,0)\right)$. Обозначим также нижнюю грань тех $\mu_{0}$, для которых устойчива фаза 0, как $\mu_{k}(\varepsilon)$. Мы предполагаем, что $\mu_{K}(\varepsilon)$ совпадает с $\mu_{0, \infty}^{* *}(\varepsilon)$, но пока не доказали это утверждение. Заметим, что при $\mu_{0}<\mu_{K}(\varepsilon)$ фаза 0 не может быть устойчивой, а при $\mu_{0}>$ $\mu_{K}(\varepsilon)$ устойчива лишь фаза 0 , как это вытекает из неравенств $\left|\partial p / \partial \mu_{0}\right|<C e^{-\varepsilon_{1}}$, $\left|\partial p_{q} / \partial \mu_{0}-2\right|<C e^{-\varepsilon_{1}}, q>0$.

Сформулируем полученные результаты.

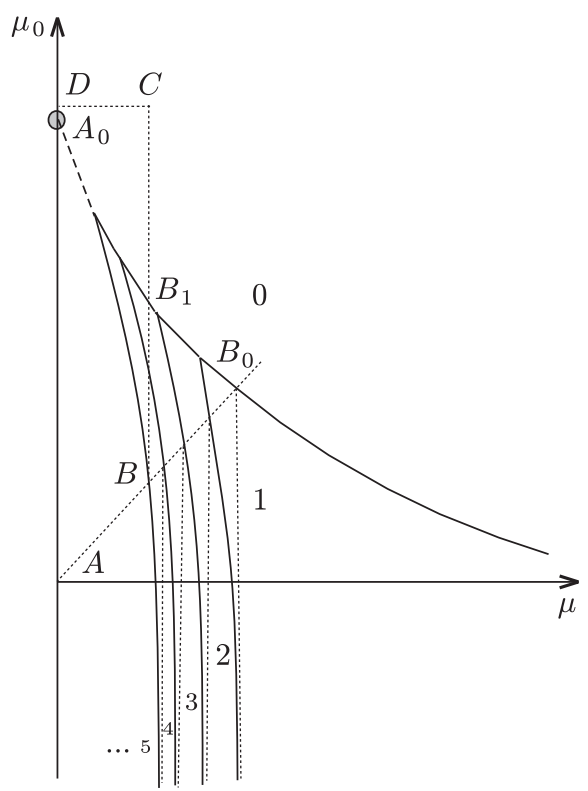

Рис. 2. Фазовая диаграмма с произвольным внешним полем в нулевом слое.

Теорема 6. 1. Для модели Изинга в полупространстве с произвольным магнитным полем в нулевом слое существует достаточно большое $\varepsilon_{0}$ такое, что в области $\mathcal{M}(81)$ термодинамический предел определяет поверхностную свободную 
энергию

$$
p\left(\varepsilon, \mu_{0}, \mu\right):=-\lim _{\substack{N \rightarrow \infty, \Lambda_{S} \uparrow \mathbb{Z}_{0}^{\nu-1}}} \frac{1}{\left|\Lambda_{S}\right|} \ln \frac{\Xi_{q}\left(\Lambda ; \mu_{0}, \mu\right) e^{\varepsilon\left|\Lambda_{S}\right|}}{\Xi_{+}(\Lambda ; \mu, \mu)} .
$$

Этот предел не зависит от $q$. Поверхностная свободная энергия совпадает с $-p\left(\varepsilon, \mu_{0}, \mu\right)=\min _{q}\left(-p_{q}\left(\varepsilon, \mu_{0}, \mu\right)\right)$, где $\overrightarrow{\mathbf{p}}\left(\varepsilon, \mu_{0}, \mu\right)$ определяется из уравнения состояния поверхностных фаз

$$
\overrightarrow{\mathbf{p}}=\overrightarrow{\mathbf{h}}+\overrightarrow{\mathbf{s}}\left(\overrightarrow{\mathbf{p}}, \varepsilon, \mu_{0}, \mu\right), \quad \overrightarrow{\mathbf{h}}=\overrightarrow{\mathbf{h}}\left(\varepsilon, \mu_{0}, \mu\right),
$$

$h_{q}\left(\varepsilon, \mu_{0}, \mu\right)$ определено в $(79)$.

2. Поверхностная свободная энергия является вещественно-аналитической функиией внутри каждого страта фазовой диаграммы

$$
\partial_{G} \mathcal{M}:=\left\{\left(\varepsilon, \mu_{0}, \mu\right) \in \mathcal{M}: p\left(\varepsilon, \mu_{0}, \mu\right)=p_{q}\left(\varepsilon, \mu_{0}, \mu\right) \quad \forall q \in G\right\}
$$

$\left(\partial_{G} \mathcal{M}\right.$ - вещественно-аналитическое множество); ее производные продолжаются до непрерывных функций в замыкании каждого страта $\partial_{q} \mathcal{M}$, а предельные значения аналитичны на $\partial_{G} \mathcal{M}, q \in G$. Производные совпадают с соответствующими термодинамическими пределами:

$$
\begin{aligned}
\frac{\partial p}{\partial \varepsilon} & =-\lim _{\substack{N \rightarrow \infty, \Lambda_{S} \uparrow \mathbb{Z}_{0}^{\nu-1}}} \frac{1}{\left|\Lambda_{S}\right|} \sum_{[x, y] \cap \Lambda \neq \varnothing}\left(\left\langle\sigma_{x} \sigma_{y}\right\rangle_{q, \Lambda, \mu_{0}, \mu}-\left\langle\sigma_{x} \sigma_{y}\right\rangle_{+, \Lambda, \mu}\right)+1, \\
\frac{\partial p}{\partial \mu_{0}} & =\frac{1}{2} \lim _{\substack{N \rightarrow \infty, \Lambda_{S} \uparrow \mathbb{Z}_{0}^{\nu-1}}} \frac{1}{\left|\Lambda_{S}\right|} \sum_{x \in \Lambda_{S}}\left(\left\langle\sigma_{x}\right\rangle_{q, \Lambda, \mu_{0}, \mu}-\left\langle\sigma_{x}\right\rangle_{+, \Lambda, \mu}\right), \\
\frac{\partial p}{\partial \mu} & =\frac{1}{2} \lim _{\substack{N \rightarrow \infty, \Lambda_{S} \uparrow \mathbb{Z}_{0}^{\nu-1}}} \frac{1}{\left|\Lambda_{S}\right|} \sum_{x \in \Lambda \backslash \Lambda_{S}}\left(\left\langle\sigma_{x}\right\rangle_{q, \Lambda, \mu_{0}, \mu}-\left\langle\sigma_{x}\right\rangle_{+, \Lambda, \mu}\right),
\end{aligned}
$$

где $\langle\cdot\rangle_{q, \Lambda, \mu_{0}, \mu},\langle\cdot\rangle_{+, \Lambda, \mu}-$ усреднение по ансамблям со статистическими суммами $\Xi_{q}\left(\Lambda ; \mu_{0}, \mu\right), \Xi_{+}(\Lambda ; \mu, \mu)$, соответственно.

3. В замыкании $\partial_{q} \mathcal{M}$ гиббсовское состояние, построенное по граничному условию $\sigma^{q}$, является малым возмущением конфигурачии $\sigma^{q}$. При пересечении страта $\partial_{G} \mathcal{M},|G|>1$, разрьвна одна из производных (см. (82), (83)) по н или $\mu_{0}$.

4. Фазовая диаграмма модели имеет следующий вид, изображенный на рис. 2 вне трапеции $A B C D$.

А. Диаграмма при $\mu=0$ : на луче $\mu_{0} \geqslant \mu_{K}(\varepsilon)$ устойчива фаза 0 , на луче $\mu_{0}<$ $\mu_{K}(\varepsilon)$ фаза 0 неустойчива; на луче $\mu_{0} \leqslant 0$ нет устойчивых фаз.

Б. Диаграмма при $\mu \leqslant \mu_{0}$ : существует набор веществено-аналитических функиий $\mu_{q}(\varepsilon), \mu_{q}\left(\varepsilon, \mu_{0}\right), \mu_{i, j}^{*}, i, j=0,1, \operatorname{maких,~что~} 0<\mu_{q}(\varepsilon)<\mu_{q+1}(\varepsilon)$, функиия $\mu_{q}\left(\varepsilon, \mu_{0}\right)$ монотонно убывает по $\mu_{0}$ и является линией сосуществования фаз $q$, $q+1 ;$ при этом выполнено равенство $\mu_{q}(\varepsilon)=\mu_{q}\left(\varepsilon, \mu_{q}(\varepsilon)\right)$ и для $q>0$ имеет место сходимость $\mu_{q}\left(\varepsilon, \mu_{0}\right) \underset{\mu_{0} \rightarrow-\infty}{\rightarrow} \mu_{q-1}(\varepsilon) ;$ функции $\left.\mu_{q}\left(\varepsilon, \mu_{0}\right)\right|_{q=0,1,2}$ определеньь соответственно при $0<\mu_{0} \leqslant \mu_{0,0}^{*}(\varepsilon), \mu_{0} \leqslant \mu_{0,0}^{*}(\varepsilon), \mu_{0} \leqslant \mu_{1,0}^{*}(\varepsilon)$, кроме того, 
$\left.\mu_{q}\left(\varepsilon, \mu_{0}\right)\right|_{q=0} \underset{\mu_{0} \rightarrow+0}{\rightarrow} \infty ;$ функции $\left.\mu_{q}\left(\varepsilon, \mu_{0}\right)\right|_{q>2}$ определенъи при $\mu_{0} \leqslant \mu_{q}(\varepsilon) ;$ для функиий $\mu_{q}(\varepsilon)$ справедливы неравенства $\left|\mu_{q}(\varepsilon)-e^{-2(\nu-1) \varepsilon(q+1)}\right| \leqslant e^{(C-2(\nu-1) \varepsilon)(q+1)-2 \varepsilon}$, где $C$ - некоторая константа.

В. В точке $B_{0}:=\left(\mu_{0,0}^{*}, \mu_{1,0}^{*}\right)$ устойчивы фазы $0,1,2$, и в этой точке верны асимптотические разложения

$$
\begin{aligned}
& \mu_{0,0}^{*}(\varepsilon)=x^{2}+x^{3}+\frac{7}{2} x^{4}-22 \frac{1}{6} x^{6}+O\left(x^{7}\right), \\
& \mu_{1,0}^{*}(\varepsilon)=x^{4}+2 x^{5}+16 x^{6}+O\left(x^{7}\right)
\end{aligned}
$$

в точке $B_{1}:=\left(\mu_{0,1}^{*}, \mu_{1,1}^{*}\right)$ устойчивы фазы $0,2,3$, и в этой точке верны асимптотические разложения

$$
\begin{aligned}
& \mu_{0,1}^{*}(\varepsilon)=x^{2}+x^{3}+\frac{9}{2} x^{4}+2 x^{5}-8 \frac{1}{6} x^{6}+O\left(x^{7}\right), \\
& \mu_{1,1}^{*}(\varepsilon)=3 x^{6}+O\left(x^{7}\right) .
\end{aligned}
$$

Доказательство пункта 4В теоремы 4 можно получить, опираясь на низшие порядки разложений, построенные в разделе 9 .

Полное описание фазовой диаграммы $\left(\varepsilon>\varepsilon_{0}\right.$, см. рис. 1$):$ можно предполагать, что фазовая диаграмма имеет вид, представленный на рис. 2 при $\varepsilon>\varepsilon_{0}$. Кроме того, при $\mu_{0} \geqslant \mu_{0, \infty}^{* *}(\varepsilon), \mu=0$ устойчива лишь фаза 0, а при $\mu_{0}<\mu_{0, \infty}^{* *}(\varepsilon), \mu=0$ нет устойчивых фаз.

\section{8. ПРАВИЛО АНТОНОВА ДЛЯ МОДЕЛИ ИЗИНГА В $\mathbb{Z}_{+}^{\nu}$}

Обозначим свободную энергию модели Изинга в $\mathbb{Z}^{\nu}$ как $p_{V}(\varepsilon, \mu)$. Введем формально при помощи асимптотических разложений следующие функции:

$$
\begin{aligned}
\ln \Xi\left(\Lambda \mid \sigma^{+} ; \mu_{0}, \mu\right) & =p_{V}(\varepsilon, \mu)|\Lambda|-p_{S}^{+}\left(\varepsilon, \mu_{0}, \mu\right)\left|\Lambda_{S}\right|-p_{S}^{+}(\varepsilon, \mu, \mu)\left(|\partial \Lambda|-\left|\Lambda_{S}\right|\right)+o(|\partial \Lambda|), \\
\ln \Xi\left(\Lambda \mid \sigma^{q} ; \mu_{0}, \mu\right) & =p_{V}(\varepsilon, \mu)|\Lambda|-p_{S}^{-}\left(\varepsilon, \mu_{0}, \mu\right)\left|\Lambda_{S}\right|-p_{S}^{+}(\varepsilon, \mu, \mu)\left(|\partial \Lambda|-\left|\Lambda_{S}\right|\right)+o(|\partial \Lambda|), \\
\ln \Xi\left(\Lambda_{1} \mid \sigma^{q=0} ; 0,0\right) & =p_{V}(\varepsilon, 0)\left|\Lambda_{1}\right|-p_{S}^{+}(\varepsilon, 0,0)\left|\Lambda_{S}\right|-p^{\mathrm{D}}(\varepsilon)\left|\partial \Lambda_{1}\right|+o\left(\left|\partial \Lambda_{1}\right|\right),
\end{aligned}
$$

где $\Lambda$ - цилиндр в $\mathbb{Z}_{+}^{\nu}$ с основанием $\Lambda_{S} \subset \mathbb{Z}_{0}^{\nu-1}$ высоты $N, \Lambda_{1}$ - цилиндр со средним сечением $\Lambda_{S} \subset \mathbb{Z}_{0}^{\nu-1}$ высоты $2 N,|\partial \Lambda|$ - число узлов на границе. Из леммы 2 вытекает, что функции $p_{V}(\varepsilon, \mu), p_{S}^{+}(\varepsilon, \mu, \mu)$ аналитичны при $\varepsilon>\varepsilon_{0}, \mu>0$ и

$$
\begin{gathered}
p_{V}(\varepsilon, \mu)=-\nu \varepsilon-\frac{\mu}{2}+\sum_{X: \min _{1} X=0}^{0} \varphi^{0}(X), \\
p_{S}^{+}(\varepsilon, \mu, \mu)=p_{s t}^{+}(\varepsilon, \mu, \mu)-\frac{\varepsilon}{2}=-\frac{\varepsilon}{2}+\sum_{X: \min _{1} V(X) \cap \mathbb{Z}_{0}^{\nu-1}=0}^{0} \varphi^{0}(X) .
\end{gathered}
$$


Напомним также, что $p\left(\varepsilon, \mu_{0}, \mu\right)$ определена при помощи уравнения состояния (93) в замыкании области $\mathcal{M}=\left\{\left(\varepsilon, \mu_{0}, \mu\right): \varepsilon>\varepsilon_{0}, \mu>0\right\}$ и совпадает с термодинамическим пределом уравнения (92) в $\mathcal{M}$, а также на луче $\left\{\left(\varepsilon, \mu_{0}, 0\right): \varepsilon>\varepsilon_{0}, \mu_{0}>\mu_{K}(\varepsilon)\right\}$. При $\mu_{0} \leqslant 0$ величина $p\left(\varepsilon, \mu_{0}, 0\right)$ является верхней гранью величин $p_{q}\left(\varepsilon, \mu_{0}, 0\right)$, соответственно положим $\mathcal{M}_{\infty}=\left\{\left(\varepsilon, \mu_{0}, \mu\right): \varepsilon>\varepsilon_{0}, \mu=0, p_{q}\left(\varepsilon, \mu_{0}, 0\right)<p\left(\varepsilon, \mu_{0}, 0\right)\right.$ $\left.\forall q \in \mathbb{Z}_{+}\right\}$.

Сформулируем результаты, касающиеся асимптотических разложений (96)-(98), а также результаты, показывающие, как связаны различные функции в этих разложениях.

Теорема 7. 1. Асимптотические разложения (96), (97) справедливы в $\mathcal{M}$ (см. (81)), причем в этой области выполняется равенство

$$
p_{S}^{+}\left(\varepsilon, \mu_{0}, \mu\right)=p_{S}^{-}\left(\varepsilon, \mu_{0}+\varepsilon, \mu\right) .
$$

Кроме того, разложение (96) справедливо при $\mu=0, \mu_{0}>\mu_{K}(\varepsilon)$; разложение (97) выполняется при $\mu=0, \mu_{0}>\mu_{K}(\varepsilon)-\varepsilon$; равенство (99) выполнено также при $\mu=0, \mu_{0}>\mu_{K}(\varepsilon)-2 \varepsilon ;$ разложение (98) верно при $\varepsilon>\varepsilon_{0}$.

2. Функции $p\left(\varepsilon, \mu_{0}, \mu\right), p_{S}^{+}\left(\varepsilon, \mu_{0}, \mu\right), p_{S}^{-}\left(\varepsilon, \mu_{0}, \mu\right)$ могут быть продолженъ до функиий, непрерывных в замыкании $\mathcal{M}$, и для них выполнено равенство

$$
p_{S}^{-}\left(\varepsilon, \mu_{0}, \mu\right)=p\left(\varepsilon, \mu_{0}, \mu\right)+p_{S}^{+}(\varepsilon, \mu, \mu)-\varepsilon+\mu-\mu_{0} .
$$

Функиия $p_{S}^{-}\left(\varepsilon, \mu_{0}, 0\right)$ аналитична при $\mu_{0}>\mu_{K}(\varepsilon) ;$ функиия $p_{S}^{+}(\varepsilon, \mu, \mu)$ аналитична nрu $\varepsilon>\varepsilon_{0}, \mu>0$.

3. В области $\mathcal{M}_{\infty}$, которой принадлежит луч $\mu=0, \mu_{0} \leqslant 0$, выполнено правило Антонова

$$
p_{S}^{-}\left(\varepsilon, \mu_{0}, \mu\right) \underset{\mu \rightarrow+0}{\rightarrow} p^{\mathrm{D}}(\varepsilon)+p_{S}^{+}\left(\varepsilon,-\mu_{0}, 0\right) .
$$

ДокАЗАТЕЛЬСтво. Из леммы 2 и теоремы 6 получаем (96), (97). Из (96) и определений статистических сумм (1), (7) получаем второе утверждение теоремы. Из равенства $\Xi\left(\Lambda \mid \sigma^{+} ; \mu_{0}, \mu\right)=\Xi\left(\Lambda \mid \sigma^{q=0} ; \mu_{0}+\varepsilon, \mu\right)$ вытекает первое утверждение теоремы (данное равенство верно "с точностью" до граничного условия; опустим доказательство того, что подобная неточность не влияет на доказываемое утверждение). Докажем непрерывность $p\left(\varepsilon, \mu_{0}, \mu\right)$ в $\overline{\mathcal{M}}$. Заметим, что из теоремы I.5 вытекает непрерывность $p\left(\varepsilon, \mu_{0}, \mu\right)$ в тех точках пространства параметров, в которых $p$ не является точкой сгущения $p_{q}$, таким образом, $p$ непрерывна в $\mathcal{M}$. Чтобы доказать непрерывность $p$ в замыкании $M$, воспользуемся неравенствами

$$
\left|\frac{\partial p_{q}}{\partial \mu}\right| \leqslant C q, \quad|\mu| \leqslant e^{\left(C-\varepsilon_{1}\right) q}, \quad\left(\varepsilon, \mu_{0}, \mu\right) \in \partial_{q} \mathcal{M} .
$$

Эти неравенства вытекают из формул (84), (53). Возьмем две точки $\left(\varepsilon, \mu_{0}, \mu\right)$ и $\left(\varepsilon, \mu_{0}, \mu^{\prime}\right)$ и обозначим $\mu_{k}$ те точки на отрезке $\left(\mu^{\prime}, \mu\right)$, где $\left|G\left(\varepsilon, \mu_{0}, \mu\right)\right|>1$. Тогда

$$
\begin{aligned}
\left|p\left(\varepsilon, \mu_{0}, \mu\right)-p\left(\varepsilon, \mu_{0}, \mu^{\prime}\right)\right| & \leqslant \sum_{k}\left|p\left(\varepsilon, \mu_{0}, \mu_{k}\right)-p\left(\varepsilon, \mu_{0}, \mu_{k+1}\right)\right| \leqslant \\
& \leqslant \sum_{k}\left|\frac{\partial p_{q_{k}}\left(\varepsilon, \mu_{0}, \bar{\mu}_{k}\right)}{\partial \mu}\right|\left|\mu_{k}-\mu_{k+1}\right|,
\end{aligned}
$$


где $q_{k}$ - фазы между $\mu_{k}$ и $\mu_{k+1}, q_{k}$ не возрастает с ростом $\mu$, как это вытекает из леммы 10. Далее, из (101) имеем

$$
\begin{aligned}
& \left|p\left(\varepsilon, \mu_{0}, \mu\right)-p\left(\varepsilon, \mu_{0}, \mu^{\prime}\right)\right| \leqslant C \sum_{k} q_{k}\left|\mu_{k}-\mu_{k+1}\right|= \\
& =C \sum_{k: q_{k}<\ln \mu^{-1} /(\varepsilon-C)} q_{k}\left|\mu_{k}-\mu_{k+1}\right|+C \sum_{k: q_{k}>\ln \mu^{-1} /(\varepsilon-C)} q_{k}\left|\mu_{k}-\mu_{k+1}\right| \leqslant \\
& \leqslant C \mu \ln \mu^{-1 /(\varepsilon-C)+C} \sum_{k: q_{k}>\ln \mu^{-1} /(\varepsilon-C)} e^{(C-\varepsilon) q} \leqslant C_{1} \mu \ln \mu^{-1} /(\varepsilon-C),
\end{aligned}
$$

что и доказывает существование предела при $\mu \rightarrow 0$.

Докажем теперь, что предельное значение $p\left(\varepsilon, \mu_{0}, \mu\right)$ при $\mu=0$ совпадает с тем, которое можно найти из уравнения состояния, и одновременно убедимся в справедливости формулы (100). Из определения операции $T$ (см. доказательство леммы 8) следует, что $\gamma_{0} \subset T^{-1} \gamma_{1} \subset \cdots \subset T^{-q} \gamma_{q} \subset \cdots \subset \gamma:=\bigcup_{q \in \mathbb{Z}_{+}} T^{-q} \gamma_{q}$. Будем считать, что компоненты векторов $\overrightarrow{\mathbf{p}}, \overrightarrow{\mathbf{h}}$ пронумерованы целыми числами, причем $p_{q}=h_{q}=\infty$ при отрицательных $q$. Далее определим $T p_{q}=p_{q+1}$. Теперь уравнение (43) для рассматриваемой здесь модели мы можем записать так, что его правая часть задана одной и той же "функцией”, в которой в зависимости от $q$ меняются лишь аргументы, т.е. в виде

$$
\mathcal{F}_{q}(\partial)=\Upsilon\left(\partial ; \varphi_{\vec{\partial}}^{q}, \vec{\partial}^{\mathrm{ex}}=\partial, \overrightarrow{\boldsymbol{\partial}} \in \gamma, T^{q} \overrightarrow{\mathbf{p}} ; \mathcal{F}_{k+q}\right)
$$

где $\varphi_{\vec{\partial}}^{q}=\varphi_{T^{q}} \overrightarrow{\boldsymbol{\partial}}$, если $T^{q} \overrightarrow{\boldsymbol{\partial}} \in \gamma_{q}$ и $\varphi_{\overrightarrow{\boldsymbol{\partial}}}^{q}=0$ в остальных случаях. Напомним, что $\left.\varphi_{\vec{\partial}}^{q}\right|_{\mu=0}=0$, если $\overrightarrow{\boldsymbol{\partial}}=\left(\overrightarrow{\boldsymbol{\partial}}_{0}, X_{1}\right), X_{1} \neq \varnothing\left(\right.$ см. (9) и (20)). Пусть $q_{k}-$ последовательность фаз для которых $p\left(\varepsilon, \mu_{0}, 0\right)=\sup _{q} p_{q}\left(\varepsilon, \mu_{0}, 0\right)=\lim _{k \rightarrow \infty} p_{q_{k}}\left(\varepsilon, \mu_{0}, 0\right)$. Из неравенства (см. (58))

$$
\left|p_{q+l}-p_{q}\right| \leqslant l\left(C_{1} \mu e^{-2 \varepsilon}+e^{\left(C-\varepsilon_{1}\right) q_{k}}\right)
$$

вытекает, что вектор $T^{q_{k}} \overrightarrow{\mathbf{p}}\left(\varepsilon, \mu_{0}, \mu\right)$ покомпонентно стремится к $p \overrightarrow{\mathbf{1}}$, где $(\overrightarrow{\mathbf{1}})_{q}=1$. Ясно также, что величина $\varphi_{\vec{\partial}}^{q}$ стремится к величине $\varphi_{\vec{\partial}}^{\mathrm{D}}$, где $\varphi_{\vec{\partial}}^{\mathrm{D}}$ определяется при помощи гамильтониана статистической суммы (24). Таким образом, аргументы уравнения (42) имеют слабый предел при $k \rightarrow \infty$, следовательно, в силу теоремы I.4 имеет предел величина $\overrightarrow{\mathbf{s}}^{(k)}$, т.е. $\overrightarrow{\mathbf{s}}^{(k)} \underset{k \rightarrow \infty}{\rightarrow} \overrightarrow{\mathbf{s}}\left(p \overrightarrow{\mathbf{1}}, \varphi_{\vec{\partial}}^{\mathrm{D}}\right)=: s_{\infty} \overrightarrow{\mathbf{1}}$. Заметим, что (см. (24) и $(22))$

$$
h_{q} \underset{q \rightarrow \infty}{\rightarrow} h^{\mathrm{D}}+\sum_{X \subset \mathbb{Z}_{+}^{\nu}: \min _{1} X \cap \mathbb{Z}_{0}^{\nu-1}=0} \varphi^{0}(X) .
$$

Рассмотрим уравнение состояния

$$
\overrightarrow{\mathbf{p}}^{(k)}=\overrightarrow{\mathbf{h}}^{(k)}+\overrightarrow{\mathbf{s}}\left(\overrightarrow{\mathbf{p}}^{(k)}, \varphi_{\vec{\partial}}^{q_{k}}\right), \quad \mu=0, \quad \overrightarrow{\mathbf{h}}^{(k)}=T^{q_{k}} \overrightarrow{\mathbf{h}} .
$$

Предельное уравнение представляет собой, с одной стороны, уравнение на $p\left(\varepsilon, \mu_{0}, 0\right) \overrightarrow{\mathbf{1}}$, а с другой стороны, отличается от уравнения состояния модели (24) 
лишь постоянным слагаемым в $\overrightarrow{\mathbf{h}}$, откуда и следует, что

$$
p\left(\varepsilon, \mu_{0}, 0\right)=p^{\mathrm{D}}(\varepsilon)+\sum_{X \subset \mathbb{Z}_{+}^{\nu}: \min _{1} X \cap \mathbb{Z}_{0}^{\nu-1}=0} \varphi^{0}(X) .
$$

То, что предел $p\left(\varepsilon, \mu_{0}, \mu\right)$ при $\mu \rightarrow+0$ совпадает с $p(\varepsilon, 0,0)=p^{\mathrm{D}}(\varepsilon)+h_{0}^{1}(\varepsilon, 0)$, доказывается аналогично.

\section{9. НИЗКОТЕМПЕРАТУРНЫЕ РАЗЛОЖЕНИЯ С ТОЧНОСТЬЮ ДО $x^{7}$}

9.1. Поверхностная свободная энергия модели Изинга в $\mathbb{Z}^{3}$ при $\mu=0$. Уравнение состояния и соответствующие контурные уравнения в этом случае имеют вид

$$
p=s\left(\mathcal{F}_{S}\right), \quad \mathcal{F}_{S}(\partial)=\sum_{\vec{\partial}: \vec{\partial}^{e x}=\partial} \varphi_{\vec{\partial}} \Delta(\vec{\partial}) e^{-p\left|V_{\vec{\partial}}\right|},
$$

где суммирование идет по границам вида $\overrightarrow{\boldsymbol{\partial}}=\left(\Gamma_{\infty}, \varnothing\right)$ (см. формулу $(24)$ и определение 1$)$, для которых геометрическая граница $V(\vec{\partial})$ совпадает с $\partial ; \Gamma_{\infty}$ соответствует конфигурации спинов $\sigma$ в $\mathbb{Z}^{3}$, совпадающей вне некоторого $V \subset \mathbb{Z}^{3}$ с конфигурацией, равной +1 в $\mathbb{Z}_{+}^{3}$ и -1 в $\mathbb{Z}_{-}^{3}$, кроме того, конфигурации $\sigma$ соответствует лишь один контур $\Gamma_{\infty}$. Величина $\left|V_{\vec{\partial}}\right|$ есть двумерный объем носителя границы,

$$
\begin{gathered}
\Delta(\overrightarrow{\boldsymbol{\partial}})=\Delta^{-1}(V(\overrightarrow{\boldsymbol{\partial}})) \prod_{i \in l(\overrightarrow{\boldsymbol{\partial}})} \Delta\left(V_{i}(\overrightarrow{\boldsymbol{\partial}})\right), \\
\Delta(V)=\Xi_{-}\left(V, \mathcal{F}_{S}\right) e^{-s\left(\mathcal{F}_{S}\right)|V|}=\exp \left\{-\sum_{X: \min _{1} X=0}^{0} \varphi^{0}(X) \frac{|V \cap \partial V(X)|}{|V(X)|}\right\}, \\
\varphi_{\overrightarrow{\boldsymbol{\partial}}}=e^{-H_{0}(\overrightarrow{\boldsymbol{\partial}})} \varphi([\overrightarrow{\boldsymbol{\partial}}]) .
\end{gathered}
$$

Так как при $\mu=0$ гамильтониан границы инвариантен относительно трансляций вдоль вертикального направления, величи́ны $\mathcal{F}_{S}\left(\partial^{q}\right)$ для всех $q$ одинаковы. То же можно сказать и о величинах $p_{q}$. Функция Урселла $\varphi([\overrightarrow{\boldsymbol{\partial}}])$ определяется гамильтонианом (см. (21), раздел III.2 и формулу (III.38))

$$
\begin{aligned}
H_{1}(\overrightarrow{\boldsymbol{\partial}}) & :=\sum_{V \subset \mathbb{Z}_{0}^{\nu-1}}\left[W(V \mid \overrightarrow{\boldsymbol{\partial}})-\sum_{q_{1}} \frac{\left|V_{q_{1}}(\overrightarrow{\boldsymbol{\partial}}) \cap V\right|}{|V|} W\left(V \mid q_{1}\right)\right]= \\
& =\sum_{B: \min _{\vec{i}} B=0}^{0}\left[W(B \mid \overrightarrow{\boldsymbol{\partial}})-\sum_{q \in \mathbb{Z}} \frac{\left|V_{q}(\overrightarrow{\boldsymbol{\partial}}) \cap \operatorname{pr} B\right|}{|\operatorname{pr} B|} W(B \mid q)\right] .
\end{aligned}
$$

Величина $\varphi^{0}(B)=\prod_{\Gamma \in B} e^{-\varepsilon|\Gamma|} \varphi^{0}([B])$, где $\varphi^{0}([B])$ - функция Урселла, определяемая $U^{0}(B)$, есть “энергия взаимодействия" твердых сердцевин контуров $B$. Если $V$ таково, что внутри $V$ нельзя поместить другой контур, то $\Delta(V)=e^{-s|V|}$. Величина $h$ имеет следующий вид:

$$
h=-\sum_{B: \min _{\vec{i}} B=0}^{0} h\left(B \mid \partial \mathbb{Z}_{+}^{\nu}\right) \varphi^{0}(B)=-\left(2 x^{3}+7 x^{5}-\frac{1}{2} 2 x^{6}-7 x^{6}\right)=-2 x^{3}-7 x^{5}+8 x^{6},
$$


где $x^{6}$ есть вклад от второй функции Урселла при двух совпадающих контурах в $h$, $7 x^{6}-$ вклад от двух контуров, имеющих общую грань.

Заметим, что границы с несвязным носителем дают превышение необходимой нам точности. Вклад двух простейших несвязных возмущений имеет порядок $x^{2} \cdot x^{2}$, a взаимодействие - порядок $x^{3}$, т.е. для подобных слагаемых низшая степень равна 7 . Отсюда в нужном нам приближении $\mathcal{F}_{S}(\partial)=\sum_{\vec{\partial}^{\text {ex }}=\partial} e^{-H_{0}(\overrightarrow{\boldsymbol{\partial}})-H_{1}(\overrightarrow{\boldsymbol{\partial}})-p\left|V_{\overrightarrow{\boldsymbol{\partial}}}\right|-s\left|V_{\overrightarrow{\boldsymbol{\partial}}}\right|}$.

Величина $H_{1}(\overrightarrow{\boldsymbol{\partial}})$ имеет низшую степень, равную 3 , и ее вклад имеет необходимую нам точность. Таким образом, $e^{-H\left(\overrightarrow{\boldsymbol{\partial}}_{0}\right)}=x^{2} e^{-x^{3}}=x^{2}-x^{3}+\cdots, \overrightarrow{\boldsymbol{\partial}}_{0}$ - минимальное возмущение $\Gamma_{\infty}$.

Перейдем к основным вычислениям. Будем изображать узлы, охватываемые границей, в виде точек, соединяя ближайшие соседи линией. Напомним, что узлы принадлежат плоскости $\mathbb{Z}_{0}^{\nu-1}$. Если высота поверхности $\Gamma_{\infty}$ над $\Gamma_{\infty}^{0,0}$ над этими узлами равна 1 (или -1), мы эту высоту не указываем, в противном случае цифра над узлом - это высота поверхности $\Gamma_{\infty}$ над $\Gamma_{\infty}^{0,0}$. Будем действовать следующим образом. Сначала для данного порядка рисуем в указанном выше смысле возможные границы, потом подсчитываем их число, затем подсчитываем вклад от второй (для второго, третьего и четвертого порядков) и третьей (лишь для шестого порядка) функций Урселла, при помощи которых определяется $s$, и после этого указываем, чему равна поверхностная свободная энергия в данном порядке. Получаем следующее:

2-й порядок: $s^{(2)}=2 x^{2},-p^{(2)}=2 x^{2}$;

3-й порядок: возможные границы имеют вид $\bullet$,

$$
s^{(4)}=4 x^{3}, \quad-p^{(3)}=2 x^{3} ;
$$

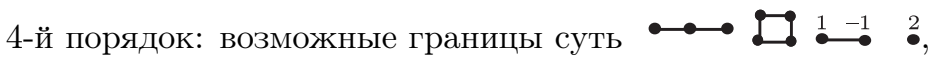

$$
s^{(4)}=x^{4}(4+8+2+4+2)-4 x^{4}(2+1 / 2)=10 x^{4}, \quad-p^{(4)}=10 x^{4} ;
$$

5-й порядок:

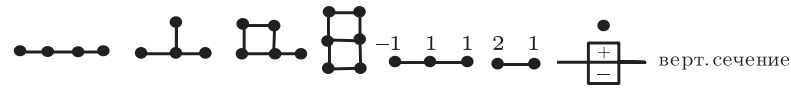

$$
\begin{aligned}
& s^{(5)}=x^{5}(28+8+16+4+24+8+1)-2 x^{5}-4 x^{5}(2+2+12)=23 x^{5} \text {, } \\
& -p^{(5)}=(23-7) x^{5}=16 x^{5} \text {; }
\end{aligned}
$$


6-й порядок: имеем

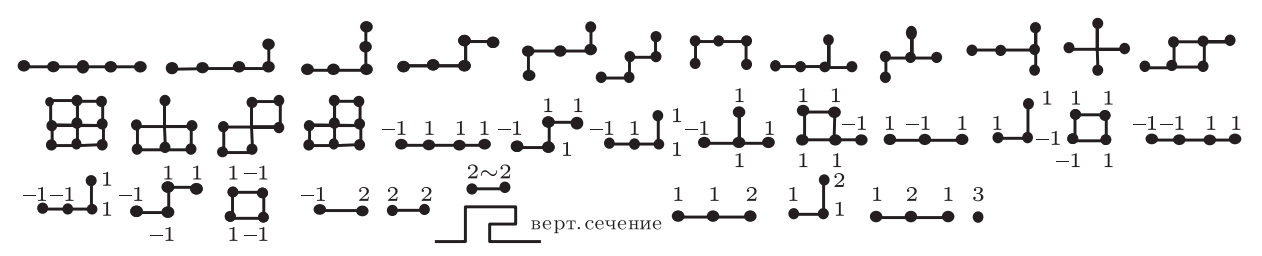

$$
\begin{gathered}
\sum \mathcal{F}^{(6)}=x^{6}(4+16+8+16+8+8+8+16+16+8+2+48+ \\
+2+8+4+8+8+16+2+8+4+8+8+16+32+24+ \\
+16+4+8+8+4+16+8+4+8+4+8+8+24+4+2), \\
\sum \mathcal{F}^{(2)} \mathcal{F}^{(4)}=380 x^{6}, \quad \sum \mathcal{F}^{(3)} \mathcal{F}^{(3)}=68 x^{6}, \quad \sum\left(\mathcal{F}^{(2)}\right)^{3}=82 \frac{2}{3} x^{6}, \\
-p^{(6)}=x^{6}\left(386-380-68+82 \frac{2}{3}+8\right)=28 \frac{2}{3} x^{6} .
\end{gathered}
$$

Итак мы нашли поверхностную свободную энергию с точностью до $x^{7}$ (см. также [22]):

$$
-p=2 x^{2}+2 x^{3}+10 x^{4}+16 x^{5}+28 \frac{2}{3} x^{6}+\cdots .
$$

9.2. Уравнение состояния модели Изинга в $\mathbb{Z}_{+}^{3}$ в низших порядках и точки сосуществования фаз 0, 1, 2 и 0, 2, 3. Из формул (79) находим

$$
\begin{aligned}
& -h_{0}^{1}=x^{3} e^{-\mu}+3 x^{5} e^{-2 \mu}-\frac{7}{2} x^{6} e^{-2 \mu}, \\
& -h_{1}^{1}=2 x^{3} e^{-\mu}+5 x^{5} e^{-2 \mu}-7 x^{6} e^{-2 \mu}, \\
& -h_{q}^{1}=2 x^{3} e^{-\mu}+7 x^{5} e^{-2 \mu}-8 x^{6} e^{-2 \mu}, \quad q>1 .
\end{aligned}
$$

Найдем теперь величины $s_{q}$ и уравнение состояния в низших порядках при помощи проведенных в пункте 9.1 вычислений. Ниже в $q$-м уравнении

$$
d_{k}=\left\{\begin{array}{ll}
e^{\Delta p_{q}+\Delta p_{k}}, & k \geqslant 0 \\
0, & k<0 ;
\end{array} \quad \Delta p_{k}=-p_{k}-\max _{l}\left(-p_{l}\right) .\right.
$$

Получаем следующее:

2-й порядок: $s_{q}^{(2)}=x^{2}\left(d_{q-1}+d_{q+1}\right)$;

3-й порядок:

$s_{q}^{(3)}=x^{3}\left(2\left(d_{q-1}^{2}+d_{q+1}^{2}\right)+e^{\Delta p_{1}-p_{\max }-(q-1) \mu} \Theta_{q, 2}+(q-2) e^{\Delta p_{1}-p_{\max }-(q-2) \mu}\left(e^{\mu}-e^{-\mu}\right) \Theta_{q, 3}\right)$,

где первое слагаемое в круглых скобках соответствует возмущению поверхности $\Gamma_{\infty}^{q, 0}$, второе слагаемое - возмущению типа $(q, \Gamma, \varnothing)$, а третье слагаемое - возмущению типа $(q, \varnothing, \Gamma)$ в (78), Г - поверхность единичного куба в $\mathbb{R}^{3}, \Theta_{q, n}=1$ при $q \geqslant n$ и $\Theta_{q, n}=0$ при $q<n$; 
4-й порядок:

$s_{q}^{(4)}=x^{4}\left(6\left(d_{q-1}^{3}+d_{q+1}^{3}\right)+4 d_{q-1} d_{q+1}+d_{q-1}^{4}+d_{q+1}^{4}+d_{q-2}+d_{q+2}-\frac{5}{2}\left(d_{q-1}^{2}+d_{q+1}^{2}\right)\right)$,

в этом порядке присутствуют только возмущения поверхности $\Gamma_{\infty}^{q, 0}$;

5-й порядок:

$$
\begin{aligned}
s_{q}^{(5)}=x^{5} & \left(18\left(d_{q-1}^{4}+d_{q+1}^{4}\right)+8\left(d_{q-1}^{5}+d_{q+1}^{5}\right)+2\left(d_{q-1}^{6}+d_{q+1}^{6}\right)+\right. \\
& +4\left(d_{q+2} d_{q+1}+d_{q-2} d_{q-1}\right)+12\left(d_{q-1} d_{q+1}^{2}+d_{q-1}^{2} d_{q+1}\right)+ \\
& +\Theta_{q, 3} e^{\Delta p_{q}-p_{\max }-(q-2) \mu}+2 \Theta_{q, 2} e^{2 \Delta p_{q}-2 p_{\max }-2(q-1) \mu}+ \\
& +(q-3) \Theta_{q, 4} e^{\Delta p_{q}-p_{\max }-(q-3) \mu}\left(e^{2 \mu}-e^{-2 \mu}\right)+ \\
& +2(q-2) \Theta_{q, 3} e^{2 \Delta p_{q}-2 p_{\max }-2(q-2) \mu}\left(e^{2 \mu}-e^{-2 \mu}\right)+ \\
& \left.\left(1-\delta_{q, 0}\right)\left(-d_{q-1}-d_{q+1}+e^{-p_{\max }+\Delta p_{q}}\right)-5 s_{q}^{(2)} s_{q}^{(3)}\right),
\end{aligned}
$$

где третья строка соответствует возмущению типа $(q, \Gamma, \varnothing)$, четвертая и пятая строки - возмущению типа $(q, \varnothing, \Gamma)$ в $(78), \Gamma$ - поверхность параллелепипеда с ребрами 1 , 1, 2; ребро длины 2 перпендикулярно плоскости проектирования;

6-й порядок:

$$
\begin{aligned}
s_{q}^{(6)}=x^{6} & \left(51\left(d_{q-1}^{5}+d_{q+1}^{5}\right)+30\left(d_{q-1}^{6}+d_{q+1}^{6}\right)+6\left(d_{q-1}^{7}+d_{q+1}^{7}\right)+4\left(d_{q-1}^{8}+d_{q+1}^{8}\right)+\right. \\
& +d_{q-1}^{9}+d_{q+1}^{9}+44\left(d_{q-1} d_{q+1}^{3}+d_{q-1}^{3} d_{q+1}\right)+8\left(d_{q-1} d_{q+1}^{4}+d_{q-1}^{4} d_{q+1}\right)+ \\
& +6\left(d_{q-1} d_{q+1}^{2}+d_{q-1}^{2} d_{q+1}\right)+32 d_{q-1}^{2} d_{q+1}^{2}+4\left(d_{q-1} d_{q+2}+d_{q-2} d_{q+1}\right)+ \\
& +2\left(d_{q-2}^{2}+d_{q+2}^{2}\right)+4\left(d_{q-2}+d_{q+2}\right) e^{-p_{\max }+\Delta p_{q}}+18\left(d_{q-1}^{2} d_{q-2}+d_{q+1}^{2} d_{q+2}\right)+ \\
& +d_{q-3}+d_{q+3}-\left(d_{q-1}+d_{q+1}\right)\left[62\left(d_{q-1}^{3}+d_{q+1}^{3}\right)+12\left(d_{q-1}^{4}+d_{q+1}^{4}\right)+\right. \\
& \left.\left.+32 d_{q-1} d_{q+1}+5\left(d_{q-2}+d_{q+2}\right)\right]-17\left(d_{q-1}^{2}+d_{q+1}^{2}\right)+\frac{31}{3}\left(d_{q-1}+d_{q+1}\right)^{3}\right) .
\end{aligned}
$$

Откуда уже нетрудно получить (94), (95).

\section{0. ЗАКЛЮЧЕНИЕ}

Перечислим характерные черты построений, реализованных в работах [36], а также в настоящей работе для модели Изинга в полупространстве.

1. Одна из целей работы [36] состояла в том, чтобы техника рассмотрения объемных фаз была той же, что и техника рассмотрения поверхностных фаз (в духе одной из идей Гиббса в его классической работе по термодинамике [1]). Для подобного "объединения" существенную роль играет единое определение многофазной контурной модели и соответствующего кластерного разложения гамильтониана границы. Все рассмотренные нами модели анализируются по введенной схеме, и, кроме того, одни и те же теоремы III.1 и III.4 применяются ко всем моделям. В работе [36] построение и анализ свойств многофазной контурной модели отделены от свойств и

5 Теоретическая и математическая физика, т. 153, № 2, 2007 г. 
анализа гамильтонианов конкретных моделей, приводящих к выполнению условий, при которых работает построенная схема.

2. Построение реализуется при выбранном векторе давления (или свободной энергии) и параметрах модели в термодинамическом пространстве $\wp=\left\{(\overrightarrow{\mathbf{p}}, \mu): \overrightarrow{\mathbf{p}} \in \mathbb{C}^{\phi}\right\}$. При этих параметрах строятся контурные уравнения и функция, задающая уравнение состояния термодинамической системы $\overrightarrow{\mathbf{h}}(\overrightarrow{\mathbf{p}}, \mu)=\overrightarrow{\mathbf{p}}-\overrightarrow{\mathbf{s}}(\overrightarrow{\mathbf{p}}, \mu)$, где $\overrightarrow{\mathbf{h}}-$ вектор внешних полей фаз модели. При построении контурных уравнений явно используется: во-первых, однородность статистических сумм относительно активностей фаз (см. раздел I.4), во-вторых, а priori предполагаемая независимость давления от граничного условия. Это позволяет сразу же перейти в термодинамическое пространство ю, в котором фазовая диаграмма является плоской.

3. Если в пространстве $ю$ фазовая диаграмма плоская, то в термодинамическом пространстве $\Im=\left\{(\overrightarrow{\mathbf{h}}, \mu): \overrightarrow{\mathbf{h}} \in \mathbb{C}^{\phi}\right\}$ фазовая диаграмма представляет собой слабое искажение плоской диаграммы и определяется уравнением состояния в стандартной форме $\overrightarrow{\mathbf{p}}=\overrightarrow{\mathbf{h}}+\overrightarrow{\mathbf{s}}(\overrightarrow{\mathbf{p}}, \mu), \overrightarrow{\mathbf{h}} \in \mathbb{C}^{\phi}$. Если же компоненты $\overrightarrow{\mathbf{h}}$ нельзя считать независимыми, то уравнение состояния, определяющее фазовую диаграмму, принимает вид $\overrightarrow{\mathbf{p}}(\mu)=\overrightarrow{\mathbf{p}}(\overrightarrow{\mathbf{h}}(\mu), \mu)$, и, следовательно, фазовая диаграмма является сечением фазовой диаграммы, близкой к плоской. В этой ситуации естественное расширение пространства параметров модели до пространства $\Im$ достаточно удобно и важно. Анализ в представленной последовательности рассуждений и в представленном виде уравнений, определяющих фазовую диаграммы, нам кажется наиболее близким к принятому в термодинамике и, надеемся, более наглядным.

4. Основную идею теории Пирогова-Синая фазовых переходов первого рода можно, в частности, сформулировать следующим образом: многофазная контурная модель с $N$ фазами эквивалентна $N$ тестовым контурным моделям с параметром таким, что внешнее распределение контуров фазы $q$ и $q$-й тестовой модели совпадают. Замечательно, что в параметрах "теста" фазовая диаграмма плоская! В работе [10] в качестве теста используются параметрические контурные модели, в работе [35] модель внешних контуров, в работе [36] (и ранее в [46]) - двухфазная симметричная контурная модель с внешним полем. От того, насколько полно известны и удобны для использования свойства тестовых моделей, зависит объем доказанных свойств рассматриваемых многофазных моделей. Во многих работах используются либо оригинальная версия Пирогова-Синая, либо версия Заградника. Использование в качестве тестовой модели двухфазной контурной модели с внешним полем дает ряд новых возможностей, что, можно надеется, подтверждает настоящая работа. Следует обратить внимание на два момента: во-первых, двухфазная контурная модель с внешним полем есть прямое обобщение модели Изинга, и область аналитичности ее по внешнему полю та же, что у модели Изинга (см. теорему 3); во-вторых, для статистических сумм с неустойчивым граничным условием двухфазной контурной модели с внешним полем нам удалось найти удачное представление (см. раздел I.4, а также лемму 1), которое существенно как в общем случае, так и при рассмотрении поверхностных фаз в рамках предложенной схемы (см. (14), (78) и определения 1 
и III.1). Поскольку этот момент является одним из ключевых в общих построениях и в построении поверхностных фаз, остановимся на нем чуть подробнее.

В разделе I.4 найдено представление для отношения неустойчивой и устойчивой статистических сумм двухфазной симметричной контурной модели с внешним полем (см. формулы (8)-(10)). Для этого отношения справедливо неравенство

$$
|\sigma(V)| \leqslant b^{V} \sum_{\substack{X: V(X) \subset V, X=\operatorname{ex} X}} \bar{F}^{X},
$$

где $\bar{F}(\Gamma)=\left|b^{-V(\Gamma)} F(\Gamma)(1-b)\right| e^{C|\Gamma|}$, которое было использовано при доказательстве теорем I.4 и теоремы III.1. Здесь следует заметить, что правая часть последнего неравенства в терминах Заградника [35] есть artificial model (без сомножителя $(1-b))$, в терминах раздела 4 - модель внешних контуров, в терминах работ [46], [36] - статистическая сумма газа “твердых объемов". Неравенство $|\sigma(V)| \leqslant 1$ является критичным в каждой из работ. Наличие сомножителя $(1-b)$, которое вытекает из алгебраических построений работ [46], [36], весьма существенно для полноты построений, тем более, что неравенство $|\sigma(V)| \leqslant 1$ верно при любом вещественном внешнем поле (или при любом давлении неустойчивой фазы в варианте, примененном к многофазной модели) и в комплексной окрестности отрезка $(0,1)$ переменной $b$. Это одно из обстоятельств, гарантирующих аналитичность давления при произвольных внешних полях фаз модели. Напомним, что это же представление (8) является одним из ключевых при рассмотрении поверхностных фаз в настоящей работе. Заметим, что (новая-старая) версия теории Пирогова-Синая, изложенная в [36], значительно упрощена по сравнению с версией работы [46] за счет алгебраических построений, связанных с введением специфических и естественных для многофазных контурных моделей функций Урселла (см. (I.14)-(I.16), (I.8)-(I.9)).

5. Как следствие построения, используемые здесь, могут быть непосредственно применены при произвольных внешних полях, не только вещественных, но и комплексных. Это почти автоматически доказывает кусочную аналитичность термодинамических функций и их производных, а также аналитичность стратов фазовой диаграммы как в случае объемных, так и в случае поверхностных фаз. Для расширения области аналитичности используется fc (field-contour)-инвариантность статистических сумм многофазных контурных моделей (см. разделы III.4 и 4).

6. Построения верны как в случае конечного, так и в случае счетного числа фаз, что существенно, в частности, при рассмотрении поверхностных фаз, например, при $\mu$, близких или равных нулю. Таким образом, нет необходимости отделять фазы с малыми активностями от фаз, близких к устойчивым, чтобы свести ситуацию к конечному числу фаз. Технически это реализовано при построении контурных уравнений в "экспоненциальной форме" и последующей записи условия Пайерлса в "экспоненциальной форме" (см. раздел III.2).

7. При анализе фазовой диаграммы можно использовать критерий устойчивости фаз - теорему III.2, если известны дополнительные неравенства на статистические суммы, в частности, корреляционные неравенства (см. раздел 7) 
8. Результаты основаны на построении сходящихся разложений для решений контурных уравнений и функций, определяющих уравнение состояния системы. При этом важно отметить относительно простую и гибкую технику формального дифференцирования мажорантных оценок в ходе доказательств. В тоже время важна возможность находить в стандартных ситуациях (когда давление не является нетривиальной точкой сгущения давлений всех фаз модели) термодинамические и корреляционные функции, а также линии (поверхности) сосуществования фаз с любой точностью (см. в качестве примера раздел 9 этой работы) в пространстве параметров модели.

9. При построении взаимодействия контуров, которое часто является многочастичным и нефинитным, например, в случае взаимодействия простейших неупорядоченных фаз $([36, \mathrm{II}])$, модели Изинга в полупространстве и т.п., существенную роль играет условие локальности теоремы I.4, а также естественное (и все же нетривиальное) определение функций Урселла гамильтониана взаимодействия контуров границы (см. определение III.2 и условия теоремы III.1), явно использующее структуру границ многофазной контурной модели. Важна также форма записи статистических сумм многофазной контурной модели в разделе I.3. При выбранной “схеме обработки" взаимодействия контуров о взаимодействии можно забыть на "некоторое время". Лишь при доказательстве теоремы III.3 было реально использовано взаимодействие контуров. С точки зрения техники важна компактная запись оценок функций Урселла с многочастичными потенциалами в лемме III.5 и использование этой записи для оценки сумм функций Урселла гамильтониана взаимодействия контуров границы.

В связи с полученным результатом можно предполагать, что для низких температур удастся доказать хорошо известное предположение об асимптотическом разложении

$$
\ln \Xi\left(\Lambda \mid \sigma^{-}\right)=p_{V}(\varepsilon, \mu)|\Lambda|-p_{S}^{-}(\varepsilon, \mu)|\partial \Lambda|-p_{l}^{-}(\varepsilon, \mu)\left|\partial^{2} \Lambda\right|-p_{0}^{-}(\varepsilon, \mu)\left|\partial^{3} \Lambda\right|+o(1),
$$

где $\Lambda$ - куб, $p_{l}^{-}(\varepsilon, \mu), p_{0}^{-}(\varepsilon, \mu)$ - гладкие функции внешнего поля. Аналитические свойства $p_{S}^{-}(\varepsilon, \mu)$ определены в настоящей работе.

Благодарности. Автор выражает искреннюю благодарность Г. П. Мещеряковой за чтение рукописи и ряд ценных замечаний по ней.

\section{Список литературы}

[1] Дж. В. Гиббс, Термодинамика. Статистическая механика, Наука, М., 1982.

[2] Y. Higuchi, "On the absence of non-translation invariant Gibbs states for the two-dimensional Ising model", Random Fields, vol. I (Esztergom, 1979), Colloq. Math. Soc. János Bolyai, 27, eds. J. Fritz, J. L. Lebowitz, D. Szász, North-Holland, Amsterdam-New York, 1981, 517-534.

[3] M. Aizenmann, Commun. Math. Phys., 73 (1980), 83-94.

[4] Р. Л. Добрушин, Теория вероятн. и ее примен., 17 (1972), 619-639.

[5] J. L. Lebowitz, J. Statist. Phys., 16 (1977), 463-476.

[6] J. Bricmont, J. L. Lebowitz, Ch. E. Pfister, E. Olivieri, Commun. Math. Phys., 66 (1979), $1-20$. 
[7] J. Bricmont, J. L. Lebowitz, Ch.E. Pfister, Commun. Math. Phys., 66 (1979), 21-36.

[8] J. Bricmont, J. L. Lebowitz, Ch. E. Pfister, Commun. Math. Phys., 69 (1979), 267-291.

[9] J. Bricmont, K. Kuroda, J. L. Lebowitz, J. Statist. Phys., 33 (1983), 59-75.

[10] С. А. Пирогов, Я. Г. Синай, ТМФ, 25 (1975), 358-369; 26 (1976), 61-76.

[11] H. van Beijeren, Commun. Math. Phys., 40 (1985), 1-6.

[12] J. L Lebowitz, Commun. Math. Phys., 35 (1974), 87-92.

[13] Д. Рюэль, Статистическая механика. Строгие результаты, Мир, М., 1971.

[14] С. Б. Шлосман, ТМФ, 66 (1986), 430-444.

[15] J. Bricmont, A. El Mellouki, J. Fröhlich, J. Statist. Phys., 46 (1986), 743-798; 47 (1987), $761-771$.

[16] J. Fröhlich, Ch.-Ed. Pfister, Commun. Math. Phys., 109 (1987), 493-523.

[17] J. Fröhlich, Ch.-Ed. Pfister, Commun. Math. Phys., 112 (1987), 51-74.

[18] J. Fröhlich, Ch.-Ed. Pfister, Europhys. Lett., 3 (1987), 845-852.

[19] Ch.-Ed. Pfister, J. Statist. Phys., 47 (1987), 761-771.

[20] Ch.-Ed. Pfister, O. Penrose, Commun. Math. Phys., 115 (1988), 691-699.

[21] А. Г. Басуев, Докл. АН СССР, 299 (1988), 854-857.

[22] В. Л. Кузьмин, ТМФ, 76 (1988), 424-431.

[23] K. Binder, "Phase transitions at surfaces", Cohesion and Structure of Surfaces, vol. 4, eds. K. Binder, M. Bowker, J. E. Inglesfield, P. J. Rous, North-Holland, Amsterdam, 1995, 121-284.

[24] L. Bahmad, A. Benyoussef, H. Ez-Zahraouy, Phys. Rev. E, 66 (2002), 056117.

[25] E. I. Dinaburg, A.E. Mazel, J. Statist. Phys., 74 (1994), 533-563.

[26] F. Cesi, F. Martinelli, J. Statist. Phys., 82 (1996), 823-913; Commun. Math. Phys., 177 (1996), 173-201.

[27] A. C. D. van Enter, R. Fernández, A. D. Sokal, J. Statist. Phys., 72 (1993), 879-1167.

[28] J. Lörinczi, Helv. Phys. Acta, 68 (1995), 605-626.

[29] P. Holický, R. Kotecký, M. Zahradnik, J. Statist. Phys., 50 (1988), 755-812.

[30] R. L. Dobrushin, R. Kotecký, S. Shlosman, Wulff construction. A global shape from local interactions, Transl. Math. Monogr., 104, AMS, Providence, RI, 1992.

[31] P. Holický, R. Kotecký, M. Zahradnik, Ann. Henri Poincaré, 3 (2002), 203-267.

[32] T. Bodineau, E. Presutti, Ann. Henri Poincaré, 4 (2003), 847-896.

[33] Y. Velenik, Probab. Theory Related Fields, 129 (2004), 83-112.

[34] Y. Velenik, Probab. Surv., 3 (2006), 112-169.

[35] M. Zahradnik, Commun. Math. Phys., 93 (1984), 559-581.

[36] А. Г. Басуев, ТМФ, 64 (1985), 103-129; 72 (1987), 255-268; 153 (2007), 98-123.

[37] F. Martinelli, E. Olivieri, R. H. Schonmann, Commun. Math. Phys., 165 (1994), 33-47.

[38] R. H. Schonmann, S. B. Shlosman, Commun. Math. Phys., 170 (1995), 453-482.

[39] R. H. Schonmann, N. Yoshida, Commun. Math. Phys., 189 (1997), 299-309.

[40] N. Yoshida, J. Statist. Phys., 90 (1998), 1015-1035.

[41] J. W. Cahn, J. Chem. Phys., 66 (1977), 3667-3672.

[42] K. Binder, "Critical behavior at surfaces", Phase Transitions and Critical Phenomena, vol. 8, eds. C. Domb, J. L. Lebowitz, Acad. Press, London, 1983, 1-144.

[43] M. E. Fisher, J. Statist. Phys., 34 (1984), 667-729.

[44] P. G. de Gennes, Rev. Modern Phys., 57 (1985), 827-863.

[45] D. B. Abraham, "Surface structure and phase transitions: Exact Results", Phase Transitions and Critical Phenomena, vol. 10, eds. C. Domb and J. L. Lebowitz, Acad. Press, London, 1986, 1.

[46] А. Г. Басуев, ТМФ, 58 (1984), 261-278.

Поступила в редакцию 29.09.2006, после доработки 20.03.2007 